\title{
PRECISION MEASUREMENT OF THE PROTON NEUTRAL WEAK
}

FORM FACTORS AT $Q^{2} \sim 0.1 \mathrm{GeV}^{2}$

A Dissertation Presented

by

LISA J. KAUFMAN

Submitted to the Graduate School of the University of Massachusetts Amherst in partial fulfillment of the requirements for the degree of

\section{DOCTOR OF PHILOSOPHY}

February 2007

Department of Physics 
(C) Copyright by Lisa J. Kaufman 2007

All Rights Reserved 


\section{PRECISION MEASUREMENT OF THE PROTON NEUTRAL WEAK FORM FACTORS AT $Q^{2} \sim 0.1 \mathrm{GeV}^{2}$}

A Dissertation Presented

by

LISA J. KAUFMAN

Approved as to style and content by:

Krishna S. Kumar, Chair

Carlo Dallapiccola, Member

Barry R. Holstein, Member

Stephen E. Schneider, Member

Jonathon L. Machta, Department Chair

Department of Physics 


\section{DEDICATION}

To my loving and supportive parents and family.

In memory of my brother. 


\section{ACKNOWLEDGMENTS}

There are many people who have provided support and guidance for me during the past few years as I embarked on this long journey. I'd first like to thank my advisor, Krishna Kumar, whose excitement for physics was contagious. I was very fortunate to have such a supportive and patient advisor who gave me the push I needed at times. He has taught me how to think like a physicist and has driven me to choose future physics experiments which are challenging and interesting to me and not just what is popular.

I also have to thank Gordon Cates for being an inspiration for how to be a physicist and make time for personal interests like music, faith, and family. His optics expertise were essential for my understanding of the polarized source. I appreciate the many conversations we've had which gave me the confidence to keep going at times. I'd also like to thank David Armstrong for being not only a great undergraduate mentor who helped realize that I wanted to do experimental nuclear and particle physics research, but who also continued as a teacher and colleague on HAPPEX. I also have to thank Paul Souder whose critical thought about every aspect of the experiment challenged me continuously and taught me to be tough.

We could not have successfully completed HAPPEX without the incredible support from the Polarized Source Group at JLab. Matt Poelker and John Hansknecht spent many hours with us in the tunnel while we did our source setup. Joe Grames gave us continual support in the laser room test stand and injector setup for the transverse asymmetry measurement. The success of the superlattice cathode's performance was because of the hard work by Maud Baylac and Marcy Stutzman. The many accelerator physicists that worked on the accelerator optics tune to ensure good performance for HAPPEX also deserve many thanks. The Hall A technical staff deserves a special thanks for all their work in the hall and

especially for all the late night trips into the lab to reset magnets and constant repair of septum computer hardware. 
I'd like to thank everyone who took shifts on the experiment, particularly, the UMass crowd for their additional support in installing and teaching us how to use the profile scanner: Kirsten Fuoti, Peter LaViolette, James Barber, Keith Otis, Darcy Lambert, and Jason Cahoon.

The HAPPEX experiment and the local collaboration was a great environment in which to work. I am thankful to have worked with such great people: David Lhuillier, Bob Michaels, Rich Holmes, and Riad Suleiman. I especially want to thank my fellow graduate students: Ryan Snyder, Hachemi Benaoum, and Bryan Moffit. Bryan and I started on HAPPEX around the same time and finished together. Bryan invited me to join a bowling league that kept me away from the lab one night a week during the experiment. Bryan's computer expertise, friendship, and humor were a great support during my 3.5 years at JLab and continued after I returned to UMass.

I worked with Brian Humensky while he was a graduate student on E158 where he began to teach me about the polarized source. I learned what a complicated device the Pockels cell is from Brian and the lessons continued from there. Brian worked with HAPPEX as a post-doc, and his expertise and hands-on teaching in the laser room were irreplaceable, and he continues to be a resource to us when we call to ask him quastions. Brian also got me to start playing fantasy football which has kept the E158 and HAPPEX folks in touch.

There are tons of people at JLab and UMass to thank for their help throughout graduate school by providing meals and tea while doing problem sets or writing this thesis, much needed distraction with day trips all over New England, and great conversation and friendship. Thanks to all of my friends: Izabela Santos, Deniz Kaya, John Cummings, Jeff Gagnon, Özgür Yavuzcetin, Rikki Roché, Olivier Gayou, Paul King, Julie Roche, Peter Monaghan, Vince Sulkosky, Bill and Mary Haga, Katie Eck, Burcu Guner, Rob Feuerbach, Bodo Reitz, Doug Higinbotham, Marcy Stutzman, Tim Holmstrom, Keoki Seu, and Aidan Kelleher.

The UMass professional staff have not only helped me with all the graduate school paper work, but also have become my friends: Jane, Ann, Mary Ann, Mary, Kris and Margaret.

Also, I want to thank my committee members who read and provided useful feedback on this thesis: Stephen Schneider, Carlo Dallapiccola and Barry Holstein. 
A special thanks goes to my roommate Jennifer Wilkes who was been with me through the undergraduate thesis and now this one. There aren't words to describe the importance of our friendship. Jen has been there for me in the best and worst of times. Her faith has been a constant example to me in my life and reminded me of what is important in this life.

I'd also like to specially thank Kingshuk Ghosh, Paul Bourgeois, Tami Stanton, Ameya Kolarkar, and Sophia Hsu for all of their support and friendship over the years and because they've become an extended family for me.

When I went to JLab in 2002, I was going to a familiar place but beginning an unfamiliar experience. Kent Paschke was the post-doc who helped me get situated. Over the course of my time at JLab, Kent was the person to talk to for any issue regarding the experiment. He was an invaluable resource, and I learned an immeasurable amount of physics, analysis, and technical expertise from working with him. Kent also became a great friend to me, and I am very thankful for it.

Finally, I'd like to thank my family who gave me the desire to learn and always encouraged me to do what was interesting to me. Their support and love has been the constant in my life, and I am glad that they could be a part of this achievement. Thank you Mom and Dad (also for being at my defense!), Karen and Chris (also for the laptop I used to write this thesis!), Doug (whom I miss very much) and Teresa, and Debra (also for the green laser pointer!). You have made me who I am today, and I couldn't have made it (especially this last month) without you. 


\begin{abstract}
PRECISION MEASUREMENT OF THE PROTON NEUTRAL WEAK FORM FACTORS AT $Q^{2} \sim 0.1 \mathrm{GeV}^{2}$
\end{abstract}

FEBRUARY 2007

LISA J. KAUFMAN

\author{
B.S., COLLEGE OF WILLIAM AND MARY \\ M.S., UNIVERSITY OF MASSACHUSETTS AMHERST \\ Ph.D., UNIVERSITY OF MASSACHUSETTS AMHERST
}

Directed by: Professor Krishna S. Kumar

This thesis reports the HAPPEX measurement of the parity-violating asymmetry for longitudinally polarized electrons elastically scattered from protons in a liquid hydrogen target. The measurement was carried out in Hall A at Thomas Jefferson National Accelerator Facility using a beam energy $E=3 \mathrm{GeV}$ and scattering angle $\left\langle\theta_{\text {lab }}\right\rangle=6^{\circ}$. The asymmetry is sensitive to the weak neutral form factors from which we extract the strange quark electric and magnetic form factors $\left(G_{E}^{s}\right.$ and $\left.G_{M}^{s}\right)$ of the proton. The measurement was conducted during two data-taking periods in 2004 and 2005. This thesis describes the methods for controlling the helicity-correlated beam asymmetries and the analysis of the raw asymmetry. The parity-violating asymmetry has been measured to be $A_{P V}=-1.14 \pm$ 0.24 (stat) \pm 0.06 (syst) ppm at $\left\langle Q^{2}\right\rangle=0.099 \mathrm{GeV}^{2}(2004)$, and $A_{P V}=-1.58 \pm 0.12$ (stat) \pm 0.04 (syst) ppm at $\left\langle Q^{2}\right\rangle=0.109 \mathrm{GeV}^{2}$ (2005). The strange quark form factors extracted from the asymmetry are $G_{E}^{s}+0.080 G_{M}^{s}=0.030 \pm 0.025$ (stat) \pm 0.006 (syst) $\pm 0.012(\mathrm{FF})$ $(2004)$ and $G_{E}^{s}+0.088 G_{M}^{s}=0.007 \pm 0.011$ (stat) \pm 0.004 (syst) \pm 0.005 (FF) (2005). These results place the most precise constraints on the strange quark form factors and indicate little strange dynamics in the proton. 


\section{TABLE OF CONTENTS}

Page

ACKNOWLEDGMENTS $\ldots \ldots \ldots \ldots \ldots \ldots \ldots \ldots \ldots \ldots \ldots \ldots \ldots \ldots \ldots \ldots \ldots$

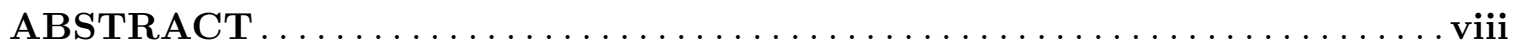

LIST OF TABLES $\ldots \ldots \ldots \ldots \ldots \ldots \ldots \ldots \ldots \ldots \ldots \ldots \ldots \ldots \ldots \ldots \ldots \ldots \ldots \ldots \ldots$

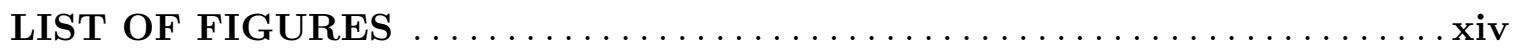

\section{CHAPTER}

1. INTRODUCTION AND FORMALISM $\ldots \ldots \ldots \ldots \ldots \ldots \ldots \ldots \ldots$

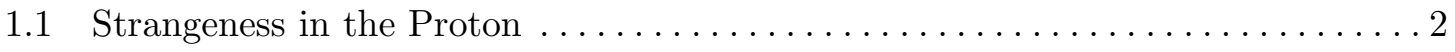

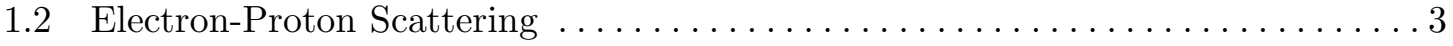

1.2.1 Electromagnetic Electron-Proton Scattering . .................. 3

1.2 .2 Weak Neutral Currents . . . . . . . . . . . . . . . . . . . . 6

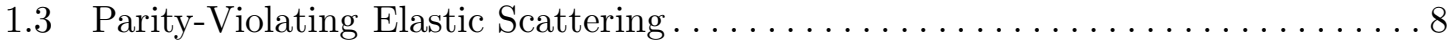

2. EXPERIMENTAL DESIGN $\ldots \ldots \ldots \ldots \ldots \ldots \ldots \ldots \ldots \ldots \ldots \ldots \ldots$

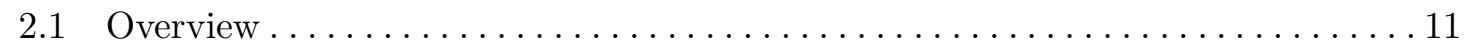

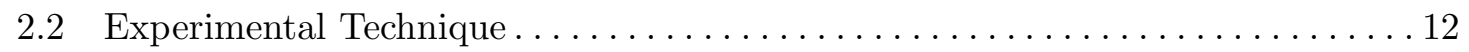

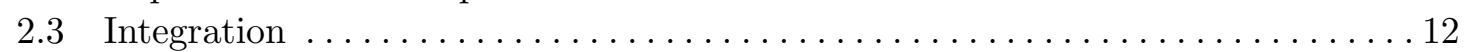

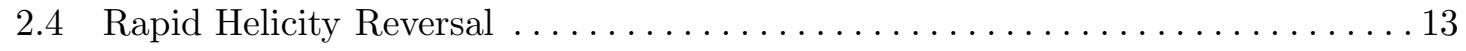

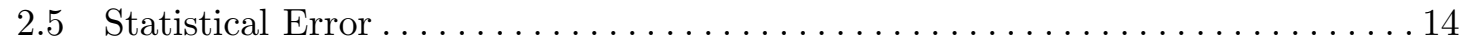

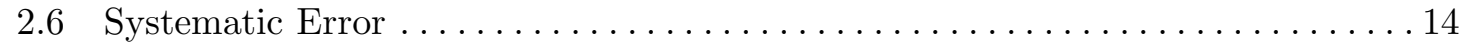

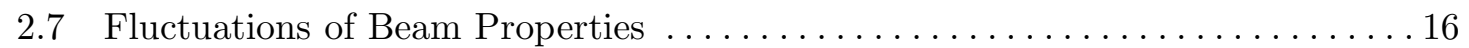

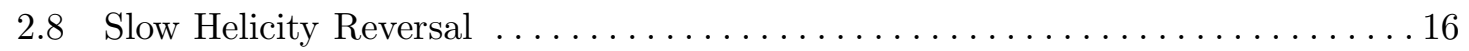

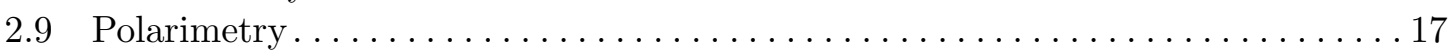

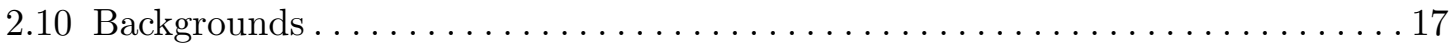

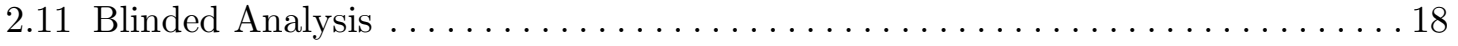

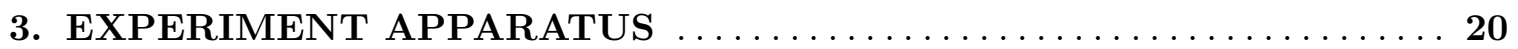

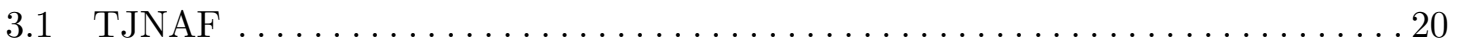

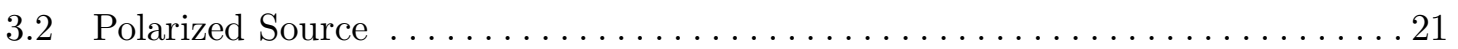




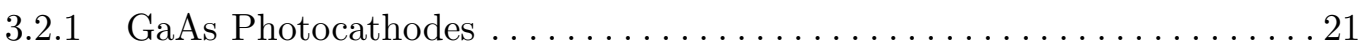

3.2.1.1 Photocathode Polarization and Quantum Efficiency ........22 22

$3.2 .1 .2 \quad$ Electron Guns............................... 24

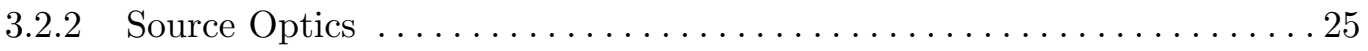

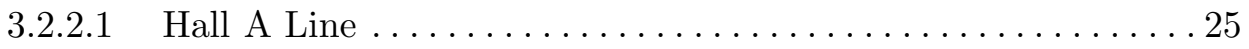

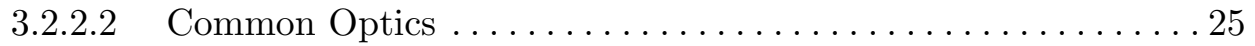

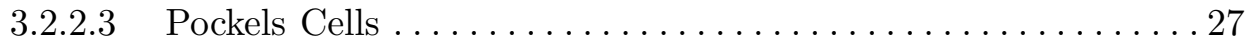

3.2.2.4 Transport to the Cathode...................... 27

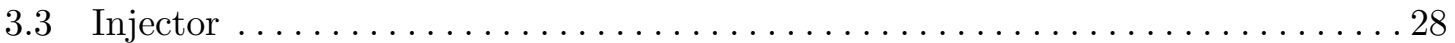

3.4 Beam Monitors and Beam Modulation . . . . . . . . . . . . . . . . . . . . 28

3.4.1 Beam Position Monitors . . . . . . . . . . . . . . . . . . . . . . 29

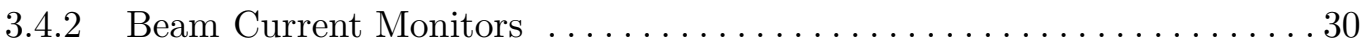

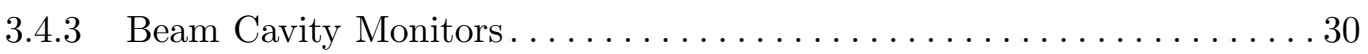

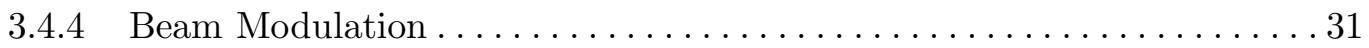

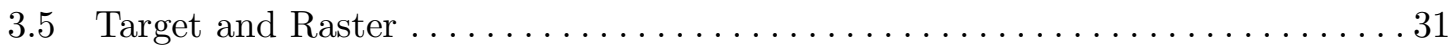

3.6 Hall A Spectrometers and Septum Magnets ....................... 33

3.6.1 High Resolution Spectrometers $\ldots \ldots \ldots \ldots \ldots \ldots \ldots \ldots \ldots \ldots \ldots \ldots \ldots$

3.6 .2 Septum Magnets................................... 35

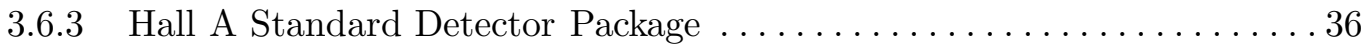

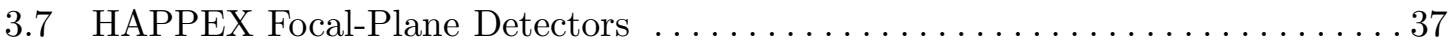

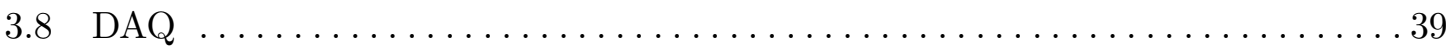

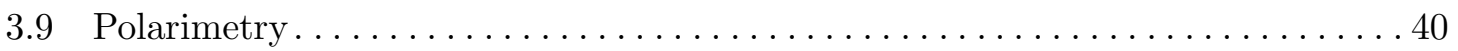

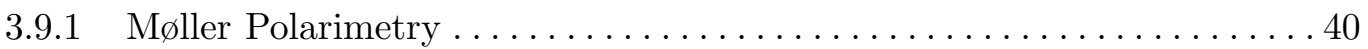

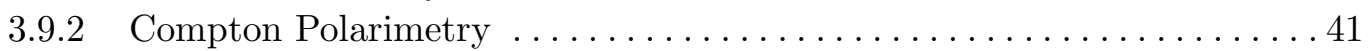

$3.10 Q^{2}$ Profile Scanners . . . . . . . . . . . . . . . . . . . . . . 41

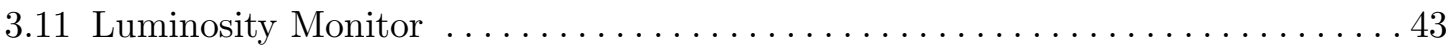

4. POLARIZED BEAM AT JEFFERSON LAB $\ldots \ldots \ldots \ldots \ldots \ldots \ldots \ldots$

4.1 Sources of Helicity-Correlated Beam Asymmetries . . . . . . . . . . . . . . . . 44

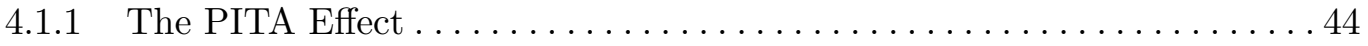

4.1.1.1 Charge Asymmetry Structure .................... 47

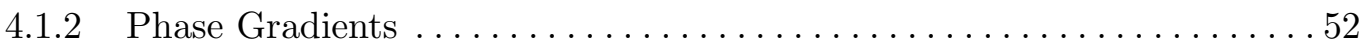

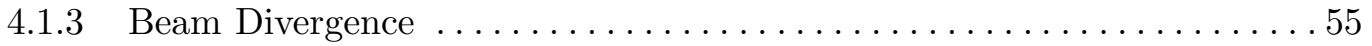

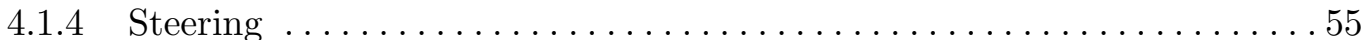

4.1.5 Cathode Analyzing Power Gradients . . . . . . . . . . . . . . 56 
4.2 Controlling Helicity-Correlated Beam Asymmetries . . . . . . . . . . . . . . . 57

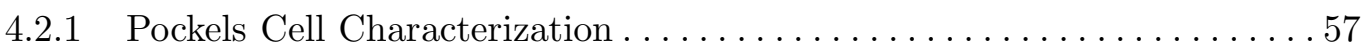

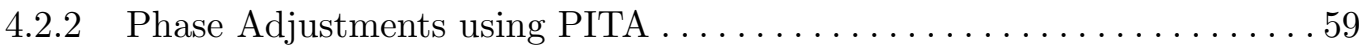

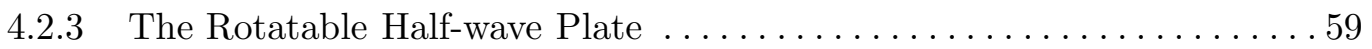

4.2.4 Minimizing Divergence Effects . . . . . . . . . . . . . . . . 61

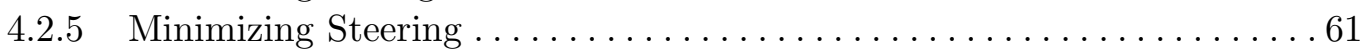

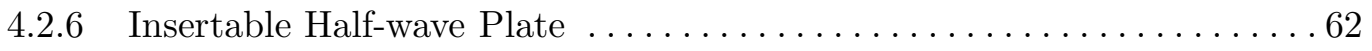

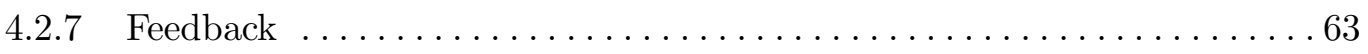

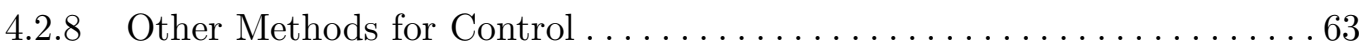

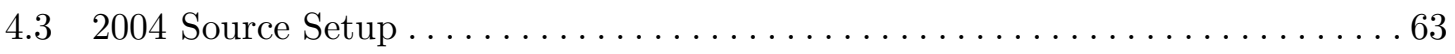

4.3.1 Beam Waist ................................ 64

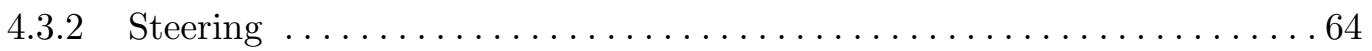

$4.3 .3 \quad 2004$ Injector Source Setup . . . . . . . . . . . . . . . . . . . 65

4.4 Helicity-Correlated Feedback . . . . . . . . . . . . . . . . . . 67

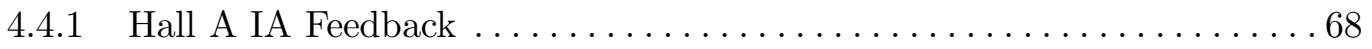

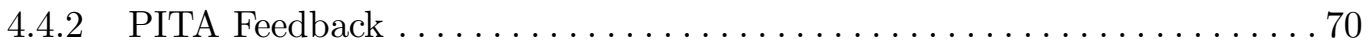

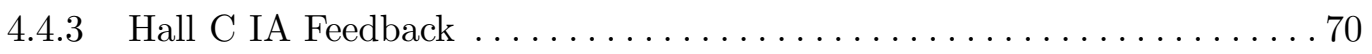

4.5 Helicity-Correlated Measurements during HAPPEX . . . . . . . . . . . . . 71

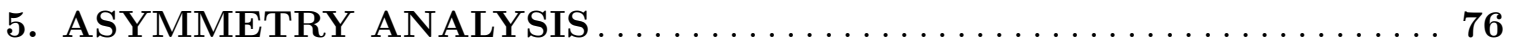

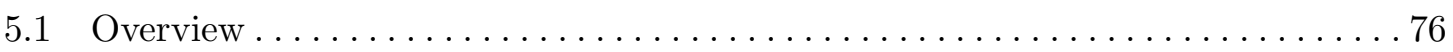

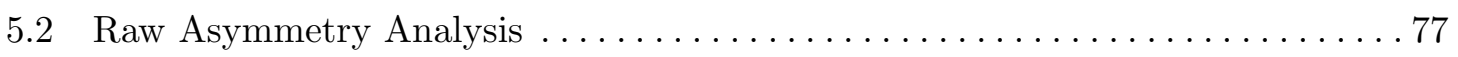

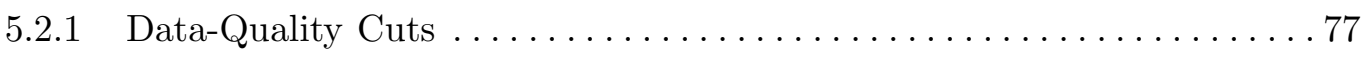

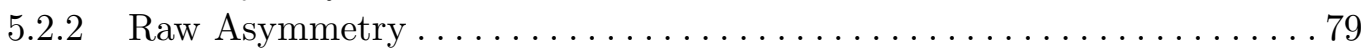

$5.2 .3 \quad$ False Asymmetry Corrections . . . . . . . . . . . . . . . . 80

5.2.3.1 Helicity-Correlated Beam Asymmetries . . . . . . . . . . . 81

5.2 .3 .2 Beam Modulation......................... 84

5.2 .3 .3 Helicity-Correlated Crosstalk . . . . . . . . . . . . . . . . 92

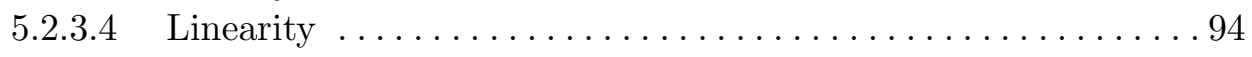

5.2.3.5 Transverse Beam Asymmetry .................... 95

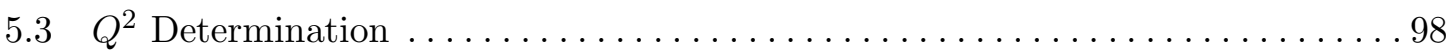

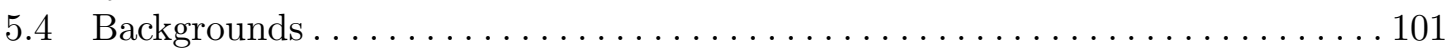

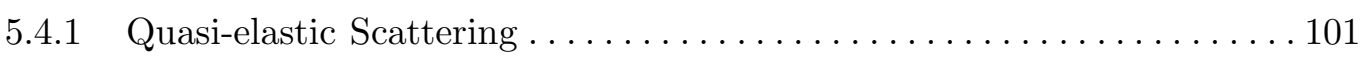

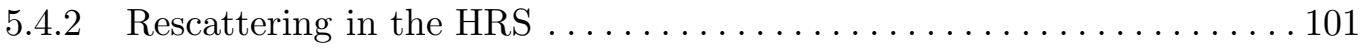

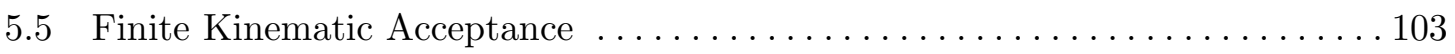

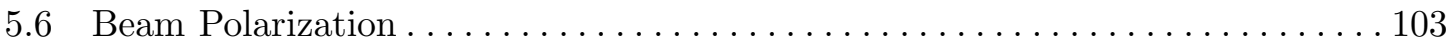

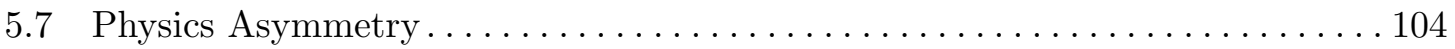




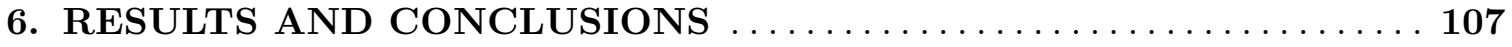

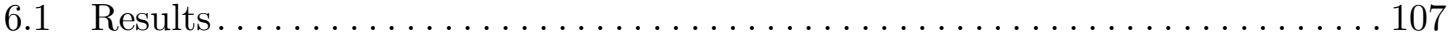

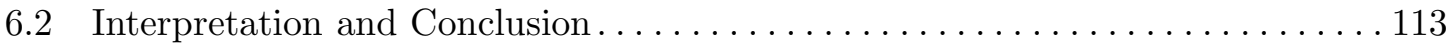

6.3 Future Measurements . . . . . . . . . . . . . . . . . . . . . . 114

6.3.1 Strange Vector Form Factors . . . . . . . . . . . . . . . . . . . . . . . . 115

$6.3 .2 \quad$ Nuclear Structure . . . . . . . . . . . . . . . . . . . . . . . . . 115

6.3.3 Physics Beyond the Standard Model. . . . . . . . . . . . . . . . . 116

APPENDIX: JLAB SOURCE CONFIGURATION $\ldots \ldots \ldots \ldots \ldots \ldots \ldots \ldots 117$

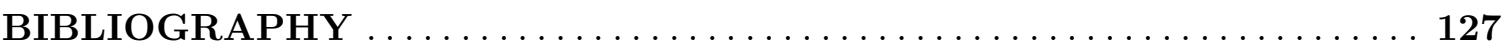




\section{LIST OF TABLES}

$\begin{array}{lll}\text { Table Page } & \text { Pag }\end{array}$

1.1 Weak charges for leptons and quarks. $\ldots \ldots \ldots \ldots \ldots \ldots \ldots \ldots \ldots \ldots \ldots \ldots \ldots \ldots \ldots \ldots \ldots \ldots$

2.1 Average helicity-correlated beam asymmetry goals. . . . . . . . . . . 15

4.1 Summary of Pockels cell characterization. $\ldots \ldots \ldots \ldots \ldots \ldots \ldots \ldots \ldots$

4.2 Average helicity-correlated beam asymmetries. . . . . . . . . . . . . 71

4.3 Locations of the beam spot on the Superlattice cathode for $2004 \ldots \ldots \ldots \ldots$. . . 72

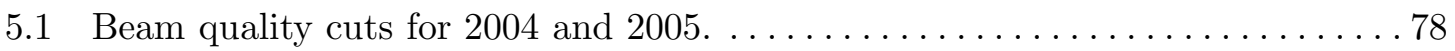

5.22005 average beam modulation slopes for each detector. . . . . . . . . . . 88

5.32005 measured position differences and corrections to $A_{\text {raw }} \ldots \ldots \ldots \ldots \ldots$

$5.42005 A_{\text {raw }}$ and $A_{\text {corr }}$ comparison for each detector combination and detector

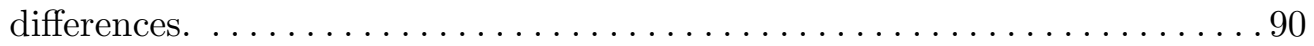

5.5 Raw and corrected asymmetries for each IHWP state . . . . . . . . . . 92

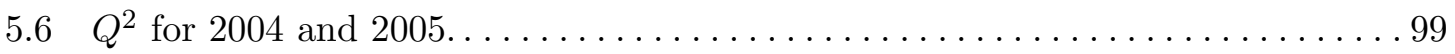

5.7 Summary of all $A_{P V}$ terms and systematic errors. ................ 105

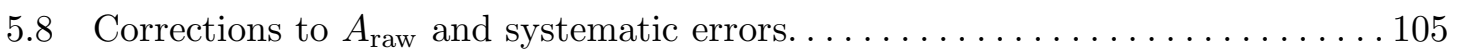

5.9 Summary of contributions to the $A_{P V}$ systematic error. ............. 106

6.1 Electroweak radiative corrections for the $A_{P V}^{(s=0)}$ calculation. . . . . . . . . 108

6.2 Values of kinematic factors used for the $A_{P V}^{(s=0)}$ calculation. $\ldots \ldots \ldots \ldots \ldots 108$

6.3 Values of the form factors used for the $A_{P V}^{(s=0)}$ calculation. . . . . . . . . 109

6.4 Summary of $A_{P V}$ and $A_{P V}^{(s=0)}$ for 2004 and $2005 \ldots \ldots \ldots \ldots \ldots \ldots \ldots \ldots \ldots \ldots \ldots \ldots \ldots$ 


\section{LIST OF FIGURES}

$\begin{array}{lll}\text { Figure } & \text { Page }\end{array}$

1.1 Tree-level Feynman diagrams for electron-proton scattering. $\ldots \ldots \ldots \ldots \ldots \ldots 3$

2.1 An example of the pseudorandom helicity sequence............... 13

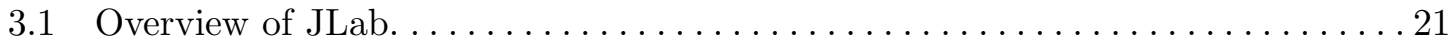

3.2 Band structure of strained GaAs............................. 22

3.3 Schematic of strained GaAs structure. $\ldots \ldots \ldots \ldots \ldots \ldots \ldots \ldots \ldots \ldots \ldots \ldots$

3.4 Superlattice cathode properties versus wavelength.................. 24

3.5 JLab source laser system for the June/July 2004 running. . . . . . . . . . . . . . 26

3.6 Schematic of beam monitor and beam modulation coil locations. . . . . . . . 29

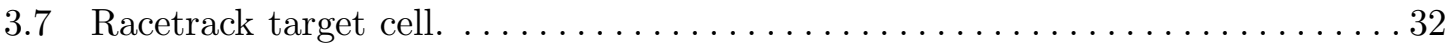

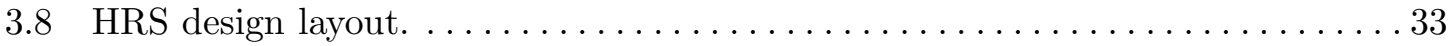

3.9 Schematic of Hall A spectrometer with the septum magnet. . . . . . . . . . 34

3.10 Elastic peak at the focal plane. . . . . . . . . . . . . . . . . . 35

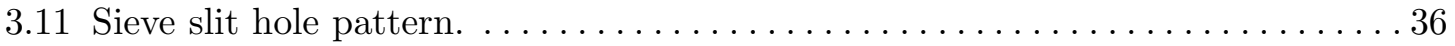

3.12 Schematic of the HAPPEX integrating detector................. 38

3.13 Schematic of the $Q^{2}$ profile scanner. $\ldots \ldots \ldots \ldots \ldots \ldots \ldots \ldots \ldots \ldots \ldots \ldots \ldots \ldots \ldots \ldots \ldots \ldots \ldots$

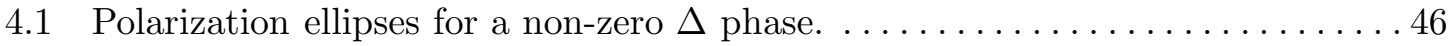

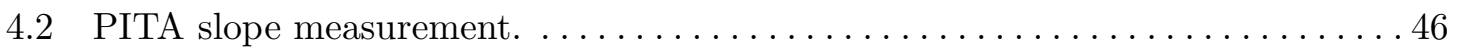

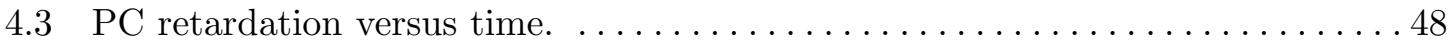

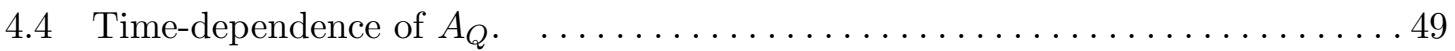




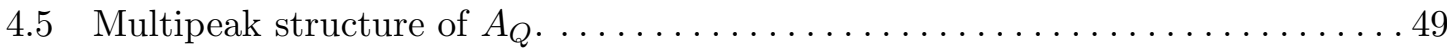

4.6 Isolated peaks of the $A_{Q}$ multipeak structure for the quad-random helicity

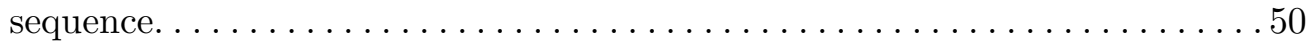

4.7 Isolated peaks of the $A_{Q}$ multipeak structure for the pair-random helicity

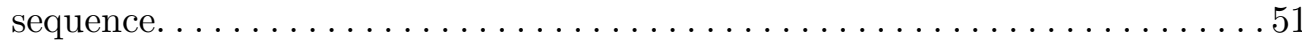

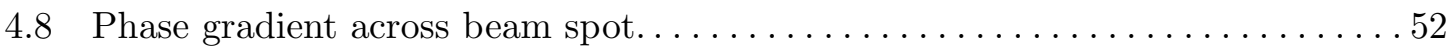

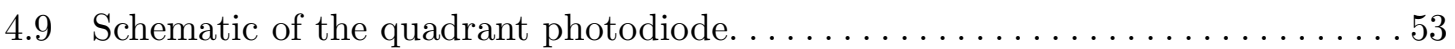

4.10 Charge asymmetry and position differences due to PC birefringence

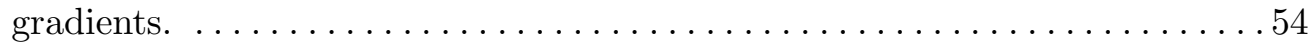

4.11 Sign flip of position differences due to phase gradients. . . . . . . . . . 54

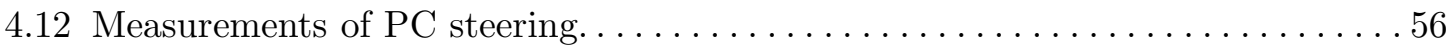

4.13 RHWP rotation of polarization ellipses. ....................... 60

4.14 Position difference response to $\mathrm{PC}$ yaw angle. . . . . . . . . . . . . 61

4.15 Steering position differences versus $\mathrm{PC}$ translation. ................. 62

$4.16 A_{Q}$ versus RHWP angle for no PITA offset.................... 66

$4.17 A_{Q}$ versus RHWP angle for a PITA offset of $-200 \mathrm{~V} \ldots \ldots \ldots \ldots \ldots \ldots \ldots$

$4.18 \Delta x$ and $\Delta y$ versus RHWP angle for no PITA offset. .............. 68

$4.19 \Delta x$ and $\Delta y$ versus RHWP angle for a PITA offset of $-200 \mathrm{~V} \ldots \ldots \ldots \ldots \ldots 9$

4.20 Superlattice and strained-layer cathode QE plots. $\ldots \ldots \ldots \ldots \ldots \ldots \ldots$

4.21 Helicity-correlated beam asymmetries versus slug for 2004 . . . . . . . . . . . . 74

4.22 Helicity-correlated beam asymmetries versus slug for 2005 . . . . . . . . . . . 75

5.12004 raw detector combination pulse-pair asymmetry distributions. . . . . . . . 8 81

5.22005 raw detector combination pulse-pair asymmetry distributions. . . . . . . . 81

5.32004 asymmetry slug plot. . . . . . . . . . . . . . . . . . . . 83

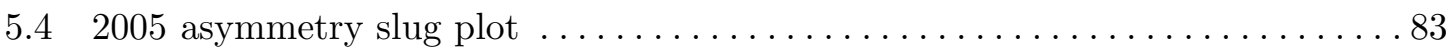

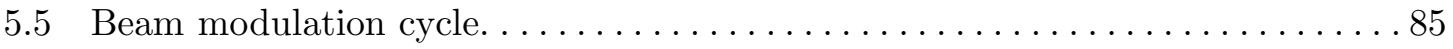


5.62004 corrected detector combination pulse-pair asymmetry distributions. . . . . 86

5.72005 corrected detector combination pulse-pair asymmetry distributions. . . . . 86

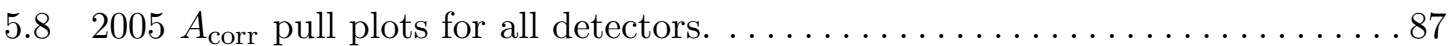

$5.92005 A_{\text {corr }}$ pull plots for detector differences. . . . . . . . . . . . . . 88

5.102005 helicity-correlated crosstalk. . . . . . . . . . . . . . . . . . . . 93

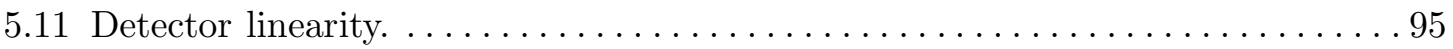

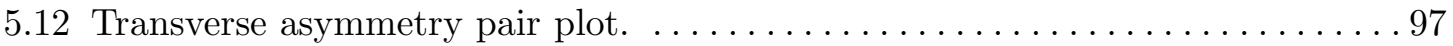

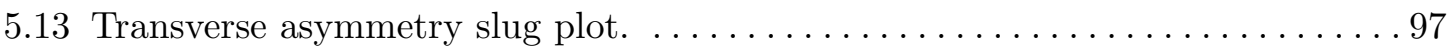

$5.142004 Q^{2}$ distributions for left and right detectors................ 100

$5.152005 Q^{2}$ scans for left and right detectors. . . . . . . . . . . . . . 100

5.16 Target window contribution to the detector flux. . . . . . . . . . . . . 102

5.172004 polarimetry results. . . . . . . . . . . . . . . . . . . . . . . . . 104

6.1 The five $A_{P V}$ measurements at $Q^{2} \sim 0.1 \mathrm{GeV}^{2}$ represented in the $G_{E}^{s}-$ $G_{M}^{s}$ plane for the 2004 HAPPEX results. . . . . . . . . . . . . . 111

6.2 The five $A_{P V}$ measurements at $Q^{2} \sim 0.1 \mathrm{GeV}^{2}$ represented in the $G_{E}^{s}-$

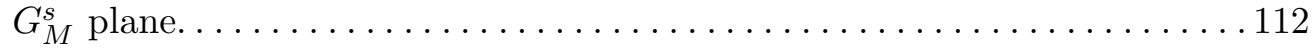

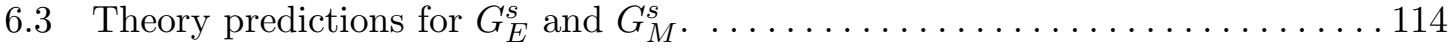

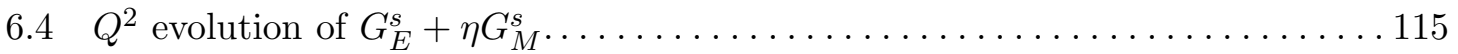

A.1 Picture of helicity Pockels cell and mount...................... 122 


\section{CHAPTER 1 INTRODUCTION AND FORMALISM}

Before 1956, it was believed that the laws of physics describing a process were the same under spatial inversion $(x \rightarrow-x)$. This mirror symmetry is called "parity conservation." Experimental evidence at the time confirmed that parity was indeed conserved in the electromagnetic and strong interactions, but there was not yet evidence for parity conservation in the weak interaction. In 1956, Lee and Yang [1] proposed several experiments to test parity conservation in the weak interaction. C. S. Wu [2] carried out one of the difficult experiments which studied beta decay of polarized ${ }^{60} \mathrm{Co}$ nuclei and found that parity was not conserved in the weak interaction and verified the process we now refer to as "parity violation."

The electroweak theory developed by Glashow, Weinberg, and Salam in the 1960s unified the electromagnetic and weak interactions and predicted the existence of the charged massive weak bosons $\left(W^{ \pm}\right.$, charged currents) and a neutral massive weak boson $\left(Z^{0}\right.$, neutral current) in addition to the known neutral massless boson $(\gamma)$. Only observations of interactions involving the charged weak currents existed before 1973 when evidence of the neutral current interaction was first found by the Gargamelle neutrino experiment at CERN [3]. Although the weak neutral current had been discovered, the electroweak model was not confirmed until 1978 when the SLAC E122 experiment measured the interference between the electromagnetic and weak neutral current amplitudes via parity-violation in inelastic electron-deuterium scattering [4].

Since the late 1970s, weak neutral current interactions have become a useful tool for testing the structure of the Standard Model and for probing the structure of the nucleon. This thesis describes the measurement of parity violation in elastic electron-proton scattering in

order to extract the strange quark contributions to the electric and magnetic properties of the proton. 


\subsection{Strangeness in the Proton}

The quark model describes baryons as strongly bound states of three valence quarks and makes predictions for their static properties based on the constituent quarks of each baryon. The proton is a baryon containing two up $(u)$ quarks and a down $(d)$ quark. While the quark model predicts the static charge of the proton, the origin of other static properties such as mass and magnetic moment remains unclear. A more complete picture of the proton takes into account the many gluons and $q \bar{q}$ pairs (the proton "sea") present due to the strong force which gives the proton a complex structure. Quantum Chromodynamics (QCD) is the theory describing the interactions of the strong force, but at low energies QCD is nonperturbative and calculations are a challenge. For example, it remains an open question to figure out at what level, if at all, the sea contributes to the proton's static properties.

The European Muon Collaboration (EMC) measured the spin-dependent structure functions of the proton via deep inelastic polarized muon scattering from polarized protons. The results [5] indicated that the valence quarks carried very little of the proton spin and generated great interest in determining the contribution of the sea, in particular the strange quarks, to the proton spin and other properties. Since then, several measurements have published results for strange quark contributions to the proton properties.

Measurements of parton distribution functions from deep-inelastic neutrino-nucleon scattering $[6,7]$ indicate that strange quarks carry a significant fraction of the proton momentum. The contribution of sea quarks to the proton spin has been measured by experiments measuring the spin-dependent structure functions in polarized deep inelastic scattering from polarized targets $[5,8]$. The contribution of strange quarks to the proton spin, $\Delta s$, could be as large as $-0.1[9]$. Measurement of the "sigma term" in pion-nucleon scattering suggest that the strange quarks could contribute $\sim 20 \%$ of the nucleon mass, but the measurements have $100 \%$ theoretical uncertainties [10,11].

Motivated by the EMC results and the pion-nucleon sigma term results, it was suggested by Kaplan and Manohar [12] to look for strange quark contributions to the nucleon vector matrix elements, i.e. the magnetic moment and charge distributions of the nucleon, by measuring the weak neutral current amplitude in elastic lepton-nucleon scattering. 


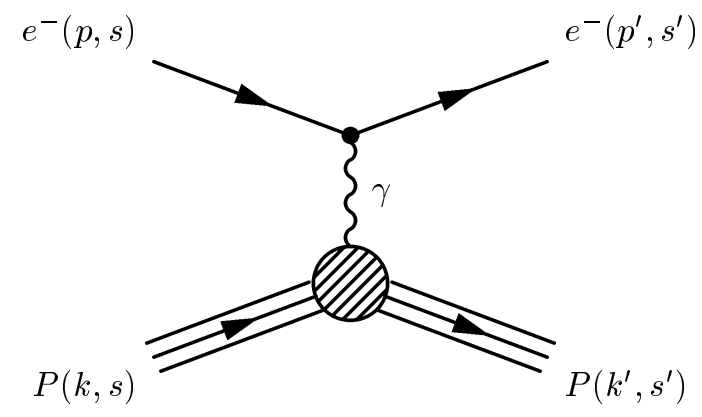

(a) 1-photon exchange

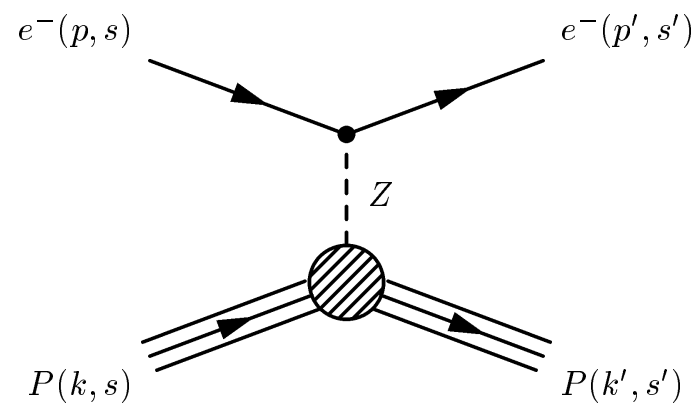

(b) $Z^{0}$ exchange

Figure 1.1. Tree-level Feynman diagrams for $e-p$ scattering.

\subsection{Electron-Proton Scattering}

In order to understand how parity-violating elastic electron-proton scattering accesses strange quarks in the nucleon, we must first develop the formalism used to describe elastic electron-proton scattering. Electrons can interact with protons through exchange of a photon, $\gamma$, or a $Z^{0}$ boson as shown in Figure 1.1. The photon is the mediator of the electromagnetic interaction while the $Z^{0}$ mediates the weak interaction, and in particular, the neutral current weak interaction. I will begin by describing the electromagnetic interaction and nucleon structure, and then I will discuss how and why we need the weak interaction to gain information about strange quarks in the nucleon.

\subsubsection{Electromagnetic Electron-Proton Scattering}

First we consider elastic electromagnetic electron-proton scattering represented by the lowest-order (tree level) Feynman diagram in Figure 1.1(a). The scattering amplitude for this process is described by the product of the electron and photon currents and the photon propagator:

$$
\mathcal{M}_{\gamma}=j_{\mu}\left(\frac{1}{q^{2}}\right) J^{\mu}
$$

where $q^{2}$ is the square of the four-momentum transfer, and the electron current is given by

$$
j_{\mu}=-e \bar{u}_{e} \gamma_{\mu} u_{e}
$$

where $e$ is the electron charge and $u_{e}\left(\bar{u}_{e}\right)$ is the incoming (outgoing) electron spinor. The proton current would be described similarly if it were a fundamental particle. Since we 
have to account for the structure of the proton, we write down the most general form of the electromagnetic current for the proton as

$$
J^{\mu}=e \bar{u}_{p}\left(F_{1}\left(q^{2}\right) \gamma^{\mu}+\frac{i}{2 M_{p}} F_{2}\left(q^{2}\right) \sigma^{\mu \nu} q_{\nu}\right) u_{p}
$$

where $F_{1}$ and $F_{2}$ are the Dirac and Pauli form factors which depend only on $q^{2}, M_{p}$ is the proton mass, and $u_{p}$ is the proton spinor. The form factors are experimentally determined quantities which describe how the proton's interactions deviate from that of a point-like particle.

It is more common to use a linear combination of $F_{1}$ and $F_{2}$ called the Sachs form factors:

$$
G_{E}^{p, n} \equiv F_{1}^{p, n}-\tau F_{2}^{p, n} \quad G_{M}^{p, n} \equiv F_{1}^{p, n}+F_{2}^{p, n}
$$

where $\tau=Q^{2} / 4 M_{p}^{2}$ and $Q^{2}=-q^{2}$. The Sachs form factors, $G_{E}^{p, n}$ and $G_{M}^{p, n}$ are referred to as the electric and magnetic form factors of the proton and neutron. In the limit of $q^{2} \ll M_{p}^{2}$, where the proton recoil is negligible, the Sachs form factors offer a physical picture of the nucleon structure as the Fourier transforms of the charge and magnetic distributions. At $Q^{2}=0$, the Sachs form factors are equal to the nucleon's charge and magnetic moment:

$$
\begin{aligned}
& G_{E}^{p}=1, \quad G_{M}^{p}=\mu_{p}=2.79, \\
& G_{E}^{n}=0, \quad G_{M}^{n}=\mu_{n}=-1.91 .
\end{aligned}
$$

The Sachs form factors combined with Equation 1.1 can be used to calculate the differential cross section for unpolarized electron-proton scattering known as the Rosenbluth formula [13]:

$$
\left.\frac{d \sigma}{d \Omega}\right|_{\mathrm{lab}}=\left(\frac{\alpha^{2}}{4 E^{2} \sin ^{4} \frac{\theta}{2}}\right) \frac{E^{\prime}}{E}\left\{\frac{\left(G_{E}^{\gamma p}\right)^{2}+\tau\left(G_{M}^{\gamma p}\right)^{2}}{1+\tau} \cos ^{2} \frac{\theta}{2}+2 \tau\left(G_{M}^{\gamma p}\right)^{2} \sin ^{2} \frac{\theta}{2}\right\}
$$

where $\alpha$ is the fine structure constant, $E$ is the incident electron energy, $E^{\prime}$ is the scattered electron energy, and $\theta$ is the lab scattering angle. The corresponding cross section for the neutron can be obtained by simply changing the superscript $p$ to $n$. Using the Sachs form 
factors ensures that there are no cross terms in the cross section formula and has enabled experimental determination of the form factors by the Rosenbluth separation method. The proton and neutron form factors have been measured over a wide range of $Q^{2}$ values [14]. The $Q^{2}$ dependence of the form factors has traditionally been parameterized by a dipole and Galster form [15] and more recently by a phenomenological fit [16] and a simple polynomial fit $[17]$.

We can understand more about the structure of the proton by describing it as a distribution of quark flavors. The proton current can be written in terms of the quark flavor currents:

$$
\begin{aligned}
J^{\mu} & =\left\langle p\left|\sum_{i=u, d, s} Q_{i} \bar{u}_{i} \gamma^{\mu} u_{i}\right| p\right\rangle \\
& =\bar{u}_{p}\left[\sum_{i=u, d, s} Q_{i}\left(F_{1}^{i} \gamma^{\mu}+\frac{i}{2 M_{p}} F_{2}^{i} \sigma^{\mu \nu} q_{\nu}\right)\right] u_{p}
\end{aligned}
$$

where $Q_{i}$ is the electric charge of quark $i$ and $u_{i}$ is the quark spinor, and $F_{1}^{i}$ and $F_{2}^{i}$ are the quark flavor form factors. We only consider the three lightest quarks, $u, d, s$, because the other quarks have mass $m_{q} \gg \Lambda_{Q C D} \cdot \Lambda_{Q C D}$ parameterizes the running of the strong coupling constant as a function of $Q^{2}$ and is empirically determined to be $\sim 0.1-0.5 \mathrm{GeV}$. For values of $Q^{2} \sim \Lambda_{Q C D}$, the strong coupling is large while for $Q^{2} \gg \Lambda_{Q C D}$, the strong coupling decreases toward "asymptotic freedom" where the quarks scarcely interact. Therefore, the contributions of the heavy quarks to proton structure are considered negligible. The $u$ and $d$ quarks are present as valence and sea quarks in the proton while the $s$ quarks are only present in the sea. For this reason, measuring the $s$ quark contributions to the proton structure offers unique access to understanding how the proton sea is involved with the proton structure.

It is more convenient to express the quark form factors in the Sachs form. Relating the proton Sachs form factors to the individual quark form factors, we get:

$$
G_{E, M}^{\gamma p}=\frac{2}{3} G_{E, M}^{u}-\frac{1}{3} G_{E, M}^{d}-\frac{1}{3} G_{E, M}^{s}
$$


The quark form factors describe the quark contributions to the proton structure. We can describe the neutron Sachs form factors with the same set of quark form factors by invoking charge symmetry. Charge symmetry is a specific rotation in isospin space about the $I_{2}$ axis such that:

$$
p \rightarrow n \quad \Rightarrow \quad u \rightarrow d, d \rightarrow u, s \rightarrow s
$$

Then the neutron Sachs form factors are written as

$$
G_{E, M}^{\gamma n}=\frac{2}{3} G_{E, M}^{d}-\frac{1}{3} G_{E, M}^{u}-\frac{1}{3} G_{E, M}^{s}
$$

Equations 1.9 and 1.11 give us two sets of linearly independent equations for the six quark form factors. In order to separate the individual quark contributions, we need a third linearly independent relationship between the form factors. Because the "weak charges" of the quarks differ from their electromagnetic charges, the weak interaction gives us this third relationship among the quark form factors and is discussed in the next section.

\subsubsection{Weak Neutral Currents}

We now consider elastic electron-proton scattering which occurs via exchange of a $Z^{0}$ boson represented by the lowest-order Feynman diagram in Figure 1.1(b). For $Q^{2} \ll M_{Z}^{2}$, the amplitude for this process is described by

$$
\mathcal{M}_{Z}=\frac{G_{F}}{2 \sqrt{2}} j_{\mu}^{Z} J^{Z, \mu}
$$

where $G_{F}$ is the Fermi coupling constant, $M_{Z}$ is the mass of the $Z^{0}$, and the electron current is

$$
j_{\mu}^{Z}=\bar{u}_{e} \gamma_{\mu}\left(g_{V}^{e}-g_{A}^{e} \gamma_{5}\right) u_{e}
$$

where $g_{V}^{e}$ and $g_{A}^{e}$ are the vector and axial-vector weak charges, respectively. The weak charges defined for each of the point-like fermions are listed in Table 1.1 in terms of the 


\begin{tabular}{|c|c|c|}
\hline Fermion & $g_{V}$ & $g_{A}$ \\
\hline \hline$\nu_{e}, \nu_{\mu}, \nu_{\tau}$ & +1 & -1 \\
$e, \mu, \tau$ & $-1+4 \sin ^{2} \theta_{W}$ & +1 \\
$u, c, t$ & $1-\frac{8}{3} \sin ^{2} \theta_{W}$ & -1 \\
$d, s, b$ & $-1+\frac{4}{3} \sin ^{2} \theta_{W}$ & +1 \\
\hline
\end{tabular}

Table 1.1. Weak charges for leptons and quarks.

electroweak mixing angle, $\theta_{W}$, which relates the weak and electromagnetic couplings to one another. We write the proton current in its general form:

$$
J^{Z, \mu}=\bar{u}_{p}\left[\gamma^{\mu} F_{1}^{Z}\left(q^{2}\right)+\frac{i}{2 M_{p}} F_{2}^{Z}\left(q^{2}\right) \sigma^{\mu \nu} q_{\nu}+\gamma^{\mu} \gamma^{5} G_{A}^{Z}+\gamma^{5} q^{\mu} F_{P}^{Z}\right] u_{p}
$$

where $F_{1}^{Z}, F_{2}^{Z}, G_{A}^{Z}$, and $F_{P}^{Z}$ are four new proton form factors which are only dependent on $Q^{2}$. The weak neutral current form factors, $F_{1}^{Z}$ and $F_{2}^{Z}$, are analogous to the $F_{1}$ and $F_{2}$ for the electromagnetic interaction. $G_{A}^{Z}$ is called the axial form factor, and $F_{P}^{Z}$ is the induced pseudoscalar form factor which vanishes when contracted with the electron current.

Similarly to what was done above, we can write the proton current as a distribution of the quarks such that:

$$
\begin{aligned}
J_{\mu}^{Z} & =\left\langle p\left|\sum_{i=u, d, s} g_{V}^{i} \bar{u}_{i} \gamma_{\mu} u_{i}\right| p\right\rangle \\
& =\bar{u}_{p}\left[\sum_{i=u, d, s} g_{V}^{i}\left(F_{1}^{i} \gamma^{\mu}+\frac{i}{2 M_{p}} F_{2}^{i} \sigma^{\mu \nu} q_{\nu}\right)\right] u_{p} .
\end{aligned}
$$

We can now write the weak neutral current form factors in terms of the quark form factors:

$$
G_{E, M}^{Z p}=\left(1-\frac{8}{3} \sin ^{2} \theta_{W}\right) G_{E, M}^{u}+\left(-1+\frac{4}{3} \sin ^{2} \theta_{W}\right)\left(G_{E, M}^{d}+G_{E, M}^{s}\right) .
$$

Because the quark-nucleon currents for both the electromagnetic and weak interactions depend only on the fact that there they interact via a vector current, the quark form factors of the two interactions are equivalent. 
Combining the results from Equations 1.16, 1.9, and 1.11, we can separate the individual quark contributions to proton structure:

$$
\begin{aligned}
& G_{E, M}^{u}=\left(3-4 \sin ^{2} \theta_{W}\right) G_{E, M}^{\gamma p}-G_{E, M}^{Z p} \\
& G_{E, M}^{d}=\left(2-4 \sin ^{2} \theta_{W}\right) G_{E, M}^{\gamma p}+G_{E, M}^{\gamma n}-G_{E, M}^{Z p} \\
& G_{E, M}^{s}=\left(1-4 \sin ^{2} \theta_{W}\right) G_{E, M}^{\gamma p}-G_{E, M}^{\gamma n}-G_{E, M}^{Z p} .
\end{aligned}
$$

We choose to eliminate the up and down quarks in favor of the strange quarks in order to better understand the role the quark sea plays in the electric and magnetic proton properties. Because the proton and neutron electric and magnetic form factors have been measured, we see from Equation 1.19 that by measuring the neutral weak vector form factors

of the proton, $G_{E, M}^{Z p}$, we can isolate the strange quark contributions, $G_{E, M}^{s}$, to the proton structure.

\subsection{Parity-Violating Elastic Scattering}

Parity-violating elastic electron-proton scattering provides a clean way to access the neutral current weak amplitude, and therefore, the weak neutral current form factors. The cross section for elastic electron-proton scattering is proportional to the square of the sum of the amplitudes for the two processes shown in Figure 1.1:

$$
\sigma \propto\left|\mathcal{M}_{\gamma}+\mathcal{M}_{Z}\right|^{2}
$$

The helicity of the electron beam is defined as positive for a right-handed electron, where the spin is aligned parallel to the electron momentum. The mirror-image of a right-handed electron, and therefore opposite parity, is a left-handed or negative helicity electron. In the negative helicity state, the electron spin is aligned antiparallel to the momentum direction. Because the weak interaction violates parity, the cross section is helicity dependent. The scattering amplitude for each helicity state can be written as:

$$
\mathcal{M}^{R}=\mathcal{M}_{\gamma}+\mathcal{M}_{Z}^{R} \quad \mathcal{M}^{L}=\mathcal{M}_{\gamma}+\mathcal{M}_{Z}^{L}
$$


Then the parity-violating asymmetry can be calculated in terms of the scattering amplitudes (assuming $\mathcal{M}_{Z} \ll \mathcal{M}_{\gamma}$ ) as:

$$
A_{P V}=\frac{\sigma_{R}-\sigma_{L}}{\sigma_{R}+\sigma_{L}}=\frac{\left|\mathcal{M}^{R}\right|^{2}-\left|\mathcal{M}^{L}\right|^{2}}{\left|\mathcal{M}^{R}\right|^{2}+\left|\mathcal{M}^{L}\right|^{2}} \simeq \frac{\mathcal{M}_{Z}^{R}-\mathcal{M}_{Z}^{L}}{\mathcal{M}_{\gamma}} .
$$

Because of the interference between the electromagnetic and weak amplitudes, the asymmetry is proportional to the ratio of the amplitudes rather than the squares of the amplitudes. The weak amplitude becomes accessible for a parity-violating asymmetry measurement in this way, and the size of the asymmetry can be estimated by:

$$
A_{P V}=\frac{\mathcal{M}_{Z}}{\mathcal{M}_{\gamma}} \sim \frac{Q^{2}}{M_{Z}^{2}}
$$

which is of order $10^{-5}$ for $Q^{2}=0.1 \mathrm{GeV}^{2}$.

The exact amplitudes depend on the proton's electric and magnetic form factors as described above. The tree-level parity-violating asymmetry is calculated [18] to be

$$
A_{P V}=-\frac{G_{F} Q^{2}}{4 \pi \alpha \sqrt{2}}\left[\frac{\epsilon G_{E}^{\gamma p} G_{E}^{Z p}+\tau G_{M}^{\gamma p} G_{M}^{Z p}-\left(1-4 \sin ^{2} \theta_{W}\right) \epsilon^{\prime} G_{M}^{\gamma p} G_{A}^{Z p}}{\epsilon\left(G_{E}^{\gamma p}\right)^{2}+\tau\left(G_{M}^{\gamma p}\right)^{2}}\right]
$$

where $\tau=Q^{2} / 4 M_{p}^{2}, \epsilon=\left[1+2(1+\tau) \tan ^{2}(\theta / 2)\right]^{-1}$, and $\epsilon^{\prime}=\sqrt{\tau(1+\tau)\left(1-\epsilon^{2}\right)}$ are kinematic factors. We can rewrite the asymmetry in terms of the measured form factors, $G_{E, M}^{p, n}$, and the strange form factors for which we want to determine by substituting

$$
G_{E, M}^{Z p}=\left(1-4 \sin ^{2} \theta_{W}\right) G_{E, M}^{\gamma p}-G_{E, M}^{\gamma n}-G_{E, M}^{s}
$$

into Equation 1.24. The asymmetry becomes:

$$
\begin{aligned}
A_{P V}=-\frac{G_{F} Q^{2}}{4 \pi \alpha \sqrt{2}}\left[\left(1-4 \sin ^{2} \theta_{W}\right)\right. & -\frac{\epsilon G_{E}^{\gamma p}\left(G_{E}^{\gamma n}+G_{E}^{s}\right)+\tau G_{M}^{\gamma p}\left(G_{M}^{\gamma n}+G_{M}^{s}\right)}{\epsilon\left(G_{E}^{\gamma p}\right)^{2}+\tau\left(G_{M}^{\gamma p}\right)^{2}} \\
& \left.-\frac{\left(1-4 \sin ^{2} \theta_{W}\right) \epsilon^{\prime} G_{M}^{\gamma p} G_{A}^{Z p}}{\epsilon\left(G_{E}^{\gamma p}\right)^{2}+\tau\left(G_{M}^{\gamma p}\right)^{2}}\right]
\end{aligned}
$$

The asymmetry is sensitive to a linear combination of $G_{E}^{s}$ and $G_{M}^{s}$ and is most sensitive to $G_{E}^{s}$ at forward angles and $G_{M}^{s}$ at backward angles. An extensive parity-violation program 
at three accelerator facilities has been developed to measure the strange quark vector form factors. The SAMPLE experiment at MIT-Bates measures $G_{M}^{s}$ and $G_{A}^{Z p}$ at backward angles from hydrogen and deuterium targets, respectively, at $Q^{2}=0.1 \mathrm{GeV}^{2}$. The A4 experiment at the Mainz Microtron measures a linear combination of $G_{E}^{s}$ and $G_{M}^{s}$ at forward angles from a hydrogen target for $Q^{2}=0.1,2.3 \mathrm{GeV}^{2}$. The G0 experiment at Jefferson Lab measures a linear combination of $G_{E}^{s}$ and $G_{M}^{s}$ for $0.12<Q^{2}<0.8 \mathrm{GeV}^{2}$ at forward angles from a hydrogen target and $G_{M}^{s}$ and $G_{A}^{Z p}$ at backward angles from hydrogen and deuterium targets at $Q^{2}=0.23,0.6 \mathrm{GeV}^{2}$. Finally, the HAPPEX collaboration measures a linear combination of $G_{E}^{s}$ and $G_{M}^{s}$ at $Q^{2}=0.1,0.48,0.6 \mathrm{GeV}^{2}$ at forward angles from a hydrogen target and $G_{E}^{s}$ at forward angles from a helium target. This thesis describes the HAPPEX-H (hydrogen) measurement at $Q^{2}=0.1 \mathrm{GeV}^{2}$. 


\section{CHAPTER 2}

\section{EXPERIMENTAL DESIGN}

The HAPPEX experiment is designed to measure a parity-violating asymmetry of order $10^{-6}$ with a statistical error of $5 \%$. The considerations that drive the experimental design are discussed in this chapter.

\subsection{Overview}

The Hall A Proton Parity EXperiment (HAPPEX) at Jefferson Lab uses the weak interaction via parity-violating elastic electron scattering from the proton as a probe to study the strange vector matrix elements. The first generation HAPPEX measured the parityviolating asymmetry of $\sim 15$ parts per million $(\mathrm{ppm})$ at $Q^{2}=0.48 \mathrm{GeV}^{2}$ with a relative error of $6 \%$ [19], and this experiment makes a measurement of the asymmetry at $Q^{2}=0.1 \mathrm{GeV}^{2}$ of $\sim 1.5 \mathrm{ppm}$ with a relative error of $5 \%$. Thus, the measurement described in this thesis requires an order of magnitude better control of systematic effects.

The HAPPEX measurement at $Q^{2}=0.1 \mathrm{GeV}^{2}$ requires measuring the asymmetry of elastically scattered electrons at very forward angles $\left(6^{\circ}\right)$ at an energy of $3 \mathrm{GeV}$. These kinematics provide a high-precision measurement in a relatively short time period.

The measurement makes use of the high polarization $(>75 \%)$ and high current $(>35 \mu \mathrm{A})$ available at Jefferson Lab. The measured asymmetry is proportional to the product of the parity-violating asymmetry and the beam polarization; therefore, a highly polarized electron beam increases the size of the measured asymmetry and decreases the amount of data necessary to achieve the same statistical precision. In order to make a $5 \%$ measurement of the asymmetry, or $\sim 0.075 \mathrm{ppm}$, we aim to keep the systematic error much smaller. The rest of this chapter describes the experimental technique used to make this statistically precise measurement and methods to control the systematic error at this level. 


\subsection{Experimental Technique}

The experiment scatters longitudinally polarized electrons from unpolarized protons in a liquid hydrogen target. The orientation of the electron polarization parallel or antiparallel to the beam direction defines the parity of the experiment. The experiment measures the asymmetry in the cross sections for these two spin configurations and is defined as:

$$
A_{P V}=\frac{\sigma_{R}-\sigma_{L}}{\sigma_{R}+\sigma_{L}}
$$

where $\sigma_{R}\left(\sigma_{L}\right)$ is the cross section for incident electrons of right (left) helicity. When the electron spin is parallel (antiparallel) to the beam direction, it is defined as the right (left) helicity state.

For the asymmetry measurement, it is sufficient to measure a quantity linearly proportional to the cross section since any common scale factor will cancel when calculating the asymmetry. HAPPEX measures the detector flux normalized to beam current which is referred to as the normalized detector flux (for simplicity of notation, the normalized detector flux will be written as $\sigma$ from now on).

\subsection{Integration}

Counting statistics dictate that one needs of order $10^{14}$ electrons in order to achieve a statistical error of $10^{-7}$ on the asymmetry. In order to accumulate this number of events in a reasonably short period of time, the total detector rate is $\sim 110 \mathrm{MHz}$. In order to count individual electrons at this rate, the experiment would need a highly segmented detector. HAPPEX instead chooses to integrate the detected flux. A fairly simple detector design can be used for an experiment utilizing the integration technique. In our case, we use a total absorption C̆erenkov calorimeter. Integration of the detected flux also means that the DAQ does not suffer any deadtime for which there could be a potentially dangerous helicity-correlated correction. Integration is sensitive to the linearity of the detector PMTs and is discussed in Section 5.2.3.4 as well as background processes that reach the detector as discussed in Section 2.10. 


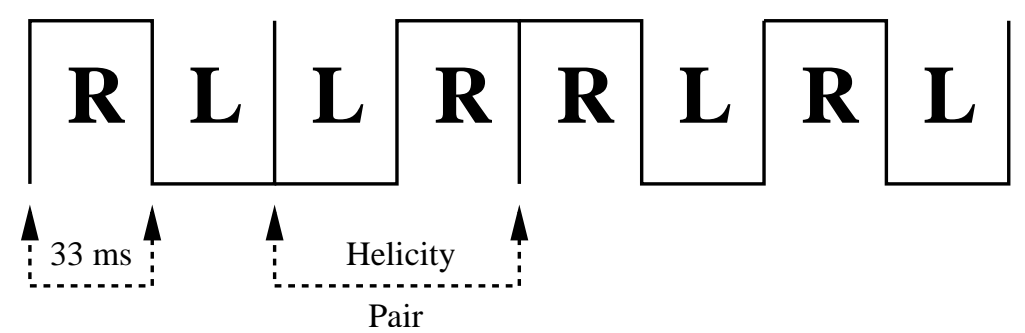

Figure 2.1. An example of the pseudorandom helicity sequence.

\subsection{Rapid Helicity Reversal}

The polarized electron source is based on photoemission from a Gallium Arsenide (GaAs) photocathode [20]. By illuminating the cathode with circularly polarized laser light, electrons are preferentially excited (depending on the sign of the polarization) from the valence band $\left(P_{3 / 2}\right)$ to one of the $s$-states of the conduction band $\left(S_{1 / 2}\right)$ due to angular momentum conservation. By reversing the sign of the laser polarization, we are able to reverse the helicity of the electron beam. We rapidly reverse the helicity of the beam in order to minimize the sensitivity of the asymmetry measurement to slow drifts in the experimental parameters. The helicity is reversed at $30 \mathrm{~Hz}$ in a pair random sequence as shown in Figure 2.1. The helicity of the first $33.3 \mathrm{~ms}$ "window" of a pair is chosen pseudorandomly by a shift-register algorithm [21], and the following window is always the complement. The asymmetry is calculated for each helicity pair. We reverse the helicity randomly so that periodic noise will not correlate to the helicity of the beam.

This helicity sequence is used as input to a high-voltage (HV) switch which applies positive or negative HV to an electro-optic device called a Pockels cell which converts linearly polarized light to right or left circularly polarized light. This light is then incident on a photocathode to produce right or left helicity electrons. This is discussed in detail in Section 3.2.

The DAQ keeps track of the helicity for each window along with the integrated detector flux and beam properties for each window. The analysis software for the experiment called 
PAN (Parity ANalyzer), using the ROOT $^{1}$ framework, stores the data for each helicity window and also forms pairs and calculates the appropriate helicity-dependent quantities.

\subsection{Statistical Error}

The integrated detector flux, $D$, is normalized to the integrated beam current, $I$, such that the raw asymmetry for each helicity pair is calculated as:

$$
A_{\text {raw }}=\frac{D_{R} / I_{R}-D_{L} / I_{L}}{D_{R} / I_{R}+D_{L} / I_{L}}
$$

The statistical error on the asymmetry for a single pair (also referred to as a pulse pair) of events is due to the counting statistics of the experiment, $1 / \sqrt{N_{R}+N_{L}}$, where $N_{R}$ and $N_{L}$ are the number of detected electrons in right and left helicity windows. For a distribution of pairs, the minimum RMS width of the distribution is equal to the statistical error on a single pair. Other sources of fluctuations can broaden the distribution of pulse-pair asymmetries.

The contributions to the broadening of the pulse-pair distribution of asymmetries occur from instrument noise in the beam position monitors and beam current monitors, ADC bit-resolution, detector pedestal fluctuations, detector energy resolution, and target density fluctuations. The goal is to keep the fluctuations from each of these sources at a level much smaller than the RMS width demanded by counting statistics. For this experiment, the largest sources of fluctuations are due to the detector energy resolution and target density fluctuations which each contributed $<2 \%$.

\subsection{Systematic Error}

Cross-section measurements of sub-ppm accuracy are unattainable, but asymmetry measurements have several advantages that make this type of accuracy possible. The advantages become clear if we consider three types of systematic errors to which the cross section is sensitive: a scale factor $S$, a common-mode offset $\Delta \sigma_{C M}$, and a helicity-correlated offset $\Delta \sigma_{H C}$ where $\Delta \sigma_{C M}, \Delta \sigma_{H C} \ll \sigma_{R(L)}$. A common-mode offset such as a slow drift in the

\footnotetext{
${ }^{1}$ ROOT documentation, http://root.cern.ch/
} 


\begin{tabular}{|c|c|}
\hline \multicolumn{2}{|c|}{ Average Helicity-correlated Beam Asymmetry Goals } \\
\hline \hline$\left\langle A_{Q}\right\rangle$ & $600 \mathrm{ppb}$ \\
$\left\langle A_{E}\right\rangle$ & $13 \mathrm{ppb}$ \\
$\langle\Delta x\rangle$ & $2 \mathrm{~nm}$ \\
$\langle\Delta y\rangle$ & $2 \mathrm{~nm}$ \\
$\left\langle\Delta x^{\prime}\right\rangle$ & $2 \mathrm{nrad}$ \\
$\left\langle\Delta y^{\prime}\right\rangle$ & $2 \mathrm{nrad}$ \\
\hline
\end{tabular}

Table 2.1. Goals for the upper limits of the average helicity-correlated beam asymmetries for the full statistics of HAPPEX-H.

PMT gain or target density contributes with the same sign to the cross section for each helicity state while a helicity-correlated offset contributes to each helicity state with opposite signs. We can write the asymmetry as:

$$
A_{\text {raw }}=\frac{S\left(\sigma_{R}+\Delta \sigma_{C M}+\Delta \sigma_{H C}\right)-S\left(\sigma_{R}+\Delta \sigma_{C M}-\Delta \sigma_{H C}\right)}{S\left(\sigma_{R}+\Delta \sigma_{C M}+\Delta \sigma_{H C}\right)+S\left(\sigma_{R}+\Delta \sigma_{C M}-\Delta \sigma_{H C}\right)}
$$

The fact that the scale factor $S$ cancels in the ratio makes it clear that an asymmetry measurement is insensitive to knowing the absolute experimental parameters such as beam current and target density which are crucial for an absolute cross-section measurement. A little algebra simplifies Equation 2.4 to

$$
A_{\text {raw }}=A_{P V}\left(1-\frac{\Delta \sigma_{C M}}{\sigma}\right)+\frac{\Delta \sigma_{H C}}{\sigma}
$$

where $\sigma=\left(\sigma_{R}+\sigma_{L}\right) / 2$ is the average normalized flux. We see that the fractional error due to a common-mode offset enters in proportion to the true asymmetry such that a $1 \%$ error in measuring the normalized flux contributes only a $1 \%$ systematic error to the measured asymmetry.

On the other hand, a systematic error due to a helicity-correlated offset contributes a systematic error independent of the size of the asymmetry. For this reason, these errors must be controlled at a level much less than the proposed statistical error as summarized in Table 2.1. The experimental efforts to control these systematics are described in detail in Chapter 4. 


\subsection{Fluctuations of Beam Properties}

Fluctuations in the beam properties such as current, position, angle, and energy on target cause fluctuations in the normalized detector flux. The helicity-correlated fluctuations in these parameters create a false asymmetry on the measured asymmetry by:

$$
A_{\text {raw }}=\frac{\Delta D}{2 D}+\frac{\Delta I}{2 I}+\beta_{E} \frac{\Delta E}{2 E}+\sum_{i} \beta_{i} \Delta x_{i}
$$

where $D$ is the average detector flux for the two helicity states, $I$ is the average current, $E$ is the average energy, $\Delta x_{i}$ are the $x$ and $y$ position and angle differences, and $\beta_{E}$ and $\beta_{i}$ are correlations between the detector flux and the energy, position, and angle. The last three terms of Equation 2.5 are the contributions to $\Delta \sigma_{H C} / \sigma$ in Equation 2.4.

We measure the helicity-correlated beam current, position, angle, and energy using beam current and position monitors along the Hall A beamline. The detector correlations to these parameters are measured using linear regression (discussed in [22]) and beam modulation (discussed in Section 5.2.3.2 and $[23,24]$ ) techniques. The beam modulation analysis is used to make reliable corrections for these helicity-correlated beam asymmetries and to determine the systematic error associated with such corrections.

\subsection{Slow Helicity Reversal}

A slow, passive reversal of the beam helicity is used in order to cancel systematic effects caused by electronic pickup of the helicity signal used for the rapid helicity flip. In addition to the beam properties fluctuating with the helicity of the beam, electronic pickup of the helicity signal by the DAQ can create a false asymmetry in the measured asymmetry.

The passive reversal is performed by inserting a half-wave plate (IHWP) into the beamline upstream of the Pockels cell. The IHWP rotates the linear polarization axis by $90^{\circ}$ such that the circular polarization produced by the Pockels cell and associated with the $R$ $(L)$ helicity signal is now opposite in sign. Because the electronic signals are unaware of the change in the electron helicity, the sign of the asymmetry calculated by the DAQ will be opposite for the two IHWP states. The sign and magnitude of any electronic pickup of the helicity will remain the same with the insertion of the IHWP and will cancel when 
averaging the asymmetries measured in the two IHWP states. Thus, this reversal scheme minimizes the false asymmetry and systematic error associated with helicity-correlated electronic pickup. This is a slow reversal because the IHWP is inserted and removed every few hours of data-taking.

\subsection{Polarimetry}

The beam polarization must be carefully measured and monitored throughout the experiment because the measured asymmetry is the parity-violating asymmetry scaled by the beam polarization. We can show this by considering the cross section as having two components: the parity-conserving piece $\sigma_{E M}$ due to the electromagnetic interaction and a parity-violating piece $\sigma_{P V}$ due to the interference of the weak and electromagnetic amplitudes. The parity-violating piece contributes with opposite sign to the cross section for each helicity state and scales with the beam polarization $P_{B}$ such that we can write $\sigma_{R}=\sigma_{E M}+\sigma_{P V}$ and $\sigma_{L}=\sigma_{E M}-\sigma_{P V}$. Then the experimental asymmetry becomes (assuming $\left.\sigma_{P V} \ll \sigma_{E M}\right)$

$$
A_{\text {raw }}=\frac{\left(\sigma_{E M}+P_{B} \sigma_{P V}\right)-\left(\sigma_{E M}-P_{B} \sigma_{P V}\right)}{\left(\sigma_{E M}+P_{B} \sigma_{P V}\right)+\left(\sigma_{E M}-P_{B} \sigma_{P V}\right)} \approx P_{B} \frac{\sigma_{P V}}{\sigma_{E M}}=P_{B} A_{P V}
$$

The systematic error in the physics asymmetry due to the beam polarization is just the fractional error in the beam polarization because the polarization contributes as a scale factor to the asymmetry. For this reason, the systematic error due to polarization is one of the dominant errors in the asymmetry. The fractional error on the polarization measurement is $1 \%$ for HAPPEX.

\subsection{Backgrounds}

The use of the integration technique for HAPPEX means that the detected flux includes the physics signal we desire to measure as well as background which cannot be separated in the data. The use of the Hall A High Resolution Spectrometers (HRS) provides a very clean separation of signal and background events at the detector location. Dedicated runs 
are performed to measure the amount of background contamination in the detected flux. The raw asymmetry can be corrected for the backgrounds by:

$$
A_{P V}=\frac{A_{\mathrm{raw}} / P_{B}-f_{\mathrm{bkg}} A_{\mathrm{bkg}}}{1-f_{\mathrm{bkg}}}
$$

where $f_{\mathrm{bkg}}$ and $A_{\mathrm{bkg}}$ are the fraction of the flux from background processes and the associated asymmetries.

The asymmetries associated with the backgrounds for HAPPEX are calculated from theory, and corrections are made as necessary. Because we have no control over the size of the background asymmetries, the fractions are kept at a sufficiently small level to keep the systematic error on the asymmetry small.

\subsection{Blinded Analysis}

A blinded asymmetry analysis is performed in order to control experimenter bias in extracting the physics asymmetry from the data. Because we are making precise measurements of small numbers, careful analysis of the detected asymmetry and all the correction terms has to be carried out. It can be tempting for an experimenter to finish the analysis for one of the correction terms when the correction achieves the "right answer" for the asymmetry. A hidden blinding offset is applied to the asymmetry in the analysis software, PAN, such that the true measured asymmetry is hidden from the experimenter to avoid this sort of bias.

A character string is provided as input to PAN and is used as an initial seed to a random number generator to produce a blinding offset $B$ with a value between -1 and 1 . The blinding offset is then scaled by a constant $C$, typically larger than the expected error on the asymmetry. The PAN database is supplied the sign of the slow reversal so that the blinding offset properly changes sign with the measured asymmetry. Thus, the blinded asymmetry used in all analyses is given by:

$$
A_{\text {blind }}=A_{\text {true }}+(-1)^{s} B C
$$


where $s$ is 0 or 1 depending on the IHWP state. The blinding factor is only removed after all analyses have been completed. 


\section{CHAPTER 3}

\section{EXPERIMENT APPARATUS}

\subsection{TJNAF}

The Thomas Jefferson National Accelerator Facility (TJNAF) in Newport News, VA is a Department of Energy facility designed to conduct research for understanding the nature of quarks and gluons inside the nucleon. The accelerator uses superconducting radio-frequency $(\mathrm{RF})$ accelerating cavities to produce a continuous-wave electron beam ${ }^{1}$ for use in the three experimental halls: A, B, and C. The beam can make up to 5 passes through the machine achieving a maximum energy of $\sim 6 \mathrm{GeV}$. The beam can be extracted for use in each experimental hall after a selected number of passes enabling the three halls to run simultaneously at three different but compatible energies. The accelerator can accommodate a beam current up to $\sim 200 \mu \mathrm{A}$. A schematic of the lab is shown in Figure 3.1.

The HAPPEX data were taken in Hall A using a polarized electron beam at an energy of $3.03 \mathrm{GeV}$ and current of $38 \mu \mathrm{A}$ for a period of 5 weeks during the summer of 2004 . In the fall of 2005, the HAPPEX data were taken with a beam energy of $3.18 \mathrm{GeV}$ and a current of $58 \mu \mathrm{A}$. When the beam arrives in Hall A, it interacts with a liquid hydrogen target and the selected scattered electrons are transported through the Hall A High Resolution Spectrometers (HRS) where they are focused into the HAPPEX detectors. There are various components along the beamline of Hall A to monitor the beam polarization, current, position, and energy in the hall. The rest of this chapter will discuss the important aspects of the accelerator and the experimental apparatus necessary to make a precision parity-violating asymmetry measurement.

\footnotetext{
${ }^{1}$ The beam is not actually continuous, but because the pulses occur at a frequency of $1497 \mathrm{MHz}$, the beam is essentially continuous.
} 


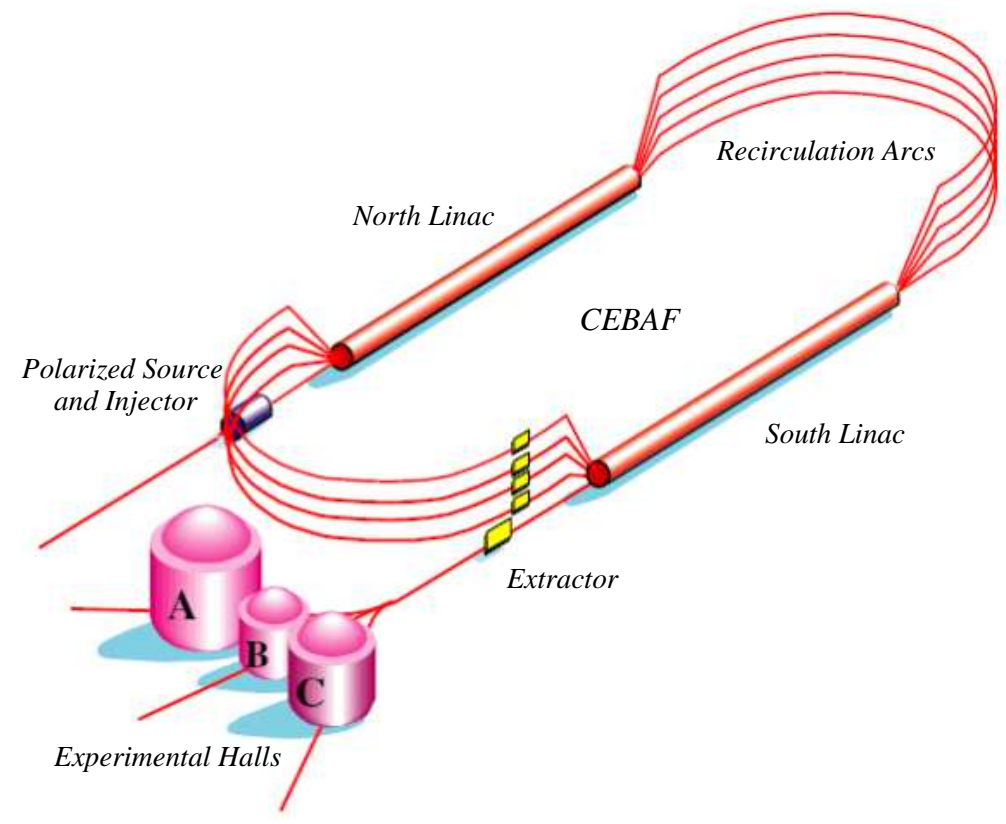

Figure 3.1. Schematic of TJNAF accelerator and experimental halls.

\subsection{Polarized Source}

The polarized electrons at JLab are produced by photoemission from a GaAs photocathode. Photons from laser light incident on the photocathode are absorbed in the crystal exciting electrons from the valence band to the conduction band. The crystal is held at a bias voltage of $-100 \mathrm{kV}$ in order to pull the electrons from the conduction band into the accelerator.

\subsubsection{GaAs Photocathodes}

The electrons are released from the cathode in a polarized state because of the properties of the crystal and laser light incident on the cathode. The crystal structure of the cathode consists of a $P_{3 / 2}$ valence band and an $S_{1 / 2}$ conduction band. Two types of "strained" GaAs cathodes were used for the 2004 HAPPEX data. The strain in the cathode is created by growing a thin layer of GaAs on a GaAsP substrate. The lattice mismatch causes the strain which breaks the four-fold degeneracy of the valence band found in "bulk" GaAs. Because of the degeneracy breaking, it is theoretically possible for the cathode to produce a $100 \%$ 


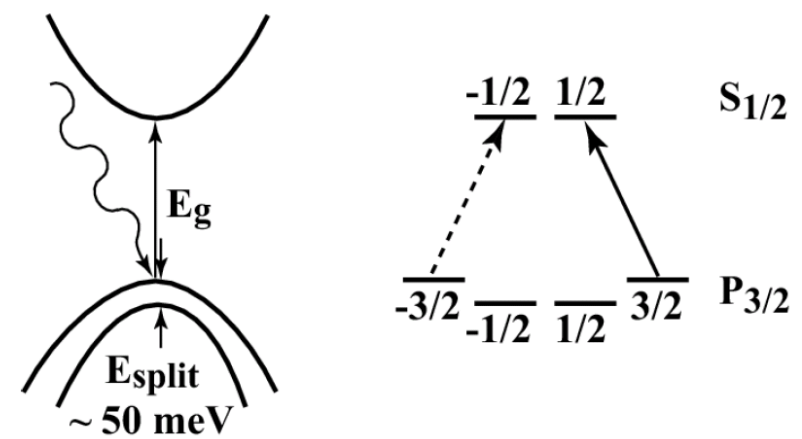

Figure 3.2. A diagram of the bandgap and energy levels for strained GaAs. The arrows indicate the allowed transitions for right- and left-helicity photons.

polarized beam of electrons when illuminated with laser light of the proper wavelength. A diagram of the energy levels and band gaps of a strained GaAs cathode can be seen in Figure 3.2. Left-circularly polarized light excites electrons into the $m_{J}=-1 / 2$ state in the conduction band while right-circularly polarized light excites electrons into the $m_{J}=+1 / 2$ state in the conduction band.

The two cathodes used for HAPPEX are described as the "strained-layer" cathode and the "superlattice" cathode. A detailed comparison of the two types of cathodes can be found in [25]. The strained-layer cathode has a $100 \mathrm{~nm}$ thick layer of GaAs grown on a 250 $\mu \mathrm{m}$ thick layer of GaAsP as shown in Figure 3.3(a). The superlattice cathode is made up of alternating layers of GaAs and GaAsP only a few nanometers thick grown on a $2.5 \mu \mathrm{m}$ thick layer of GaAsP as shown in Figure 3.3(b). HAPPEX was the first parity-violation experiment to use the superlattice cathode during the 2004 HAPPEX- ${ }^{4} \mathrm{He}$ experiment and the first week of HAPPEX-H. The average beam polarization from the superlattice cathode was measured to be $89 \%$ during HAPPEX-H. This is the highest polarization used for production running at JLab. The strained-layer cathode was used for the remaining of the HAPPEX run providing an average beam polarization of $76 \%$.

\subsubsection{Photocathode Polarization and Quantum Efficiency}

The energy gap $\mathrm{E}_{g}$ between the valence and conduction bands for the strained-layer cathode is $1.46 \mathrm{eV}$ while that for the superlattice cathode is $1.59 \mathrm{eV}$. Because of this differ- 


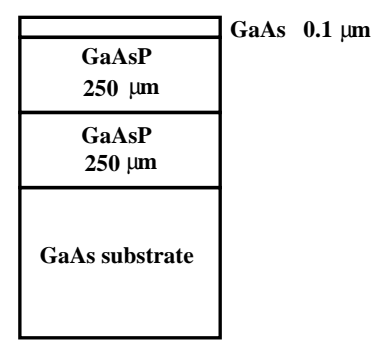

(a) Strained-layer Photocathode

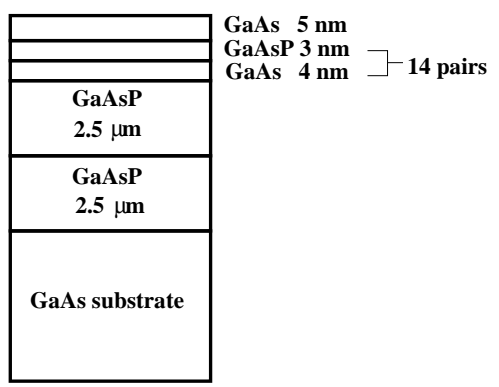

(b) Superlattice Photocathode

Figure 3.3. A schematic of the strained-layer (a) and superlattice (b) photocathode structure.

ence, two different wavelength lasers are necessary in order to produce a highly polarized electron beam from the cathodes. The source group purchased a high-power Ti-Sapphire laser from Time-Bandwidth whose optical components could be interchanged to produce a laser beam with a wavelength around $780 \mathrm{~nm}$ or $850 \mathrm{~nm}$. The laser wavelengths were optimized to provide high polarization and high quantum efficiency (QE) necessary to run HAPPEX. Quantum efficiency is defined as the number of electrons emitted from the cathode relative to the intensity of light incident on the cathode. The superlattice cathode has the advantage of providing a $\mathrm{QE}$ as high as $1 \%$ at the polarization wavelength compared to only $0.2 \%$ for the strained-layer cathode.

Plots of the polarization and $\mathrm{QE}$ as a function of wavelength for a superlattice cathode sample from the same wafer as the cathode used for HAPPEX are shown in Figure 3.4. For HAPPEX, a laser wavelength of $781 \mathrm{~nm}$ was used with the superlattice cathode and $851 \mathrm{~nm}$ with the strained-layer cathode, and the QE achieved during production running was $0.3 \%$ from the superlattice and $0.15 \%$ from the strained-layer cathode. The lack of $\mathrm{QE}$ from the superlattice was determined to be because of cathode cleaning techniques. New cleaning techniques were developed for 2005, and a QE of $0.5 \%$ was achieved.

The strain induced on the photocathode in order to produce high polarization induces a QE anisotropy [27] which means that the QE is dependent on the direction of the residual linear polarization incident on the cathode. The QE anisotropy (also called analyzing power) is different depending on the type of cathode. A typical analyzing power value for 

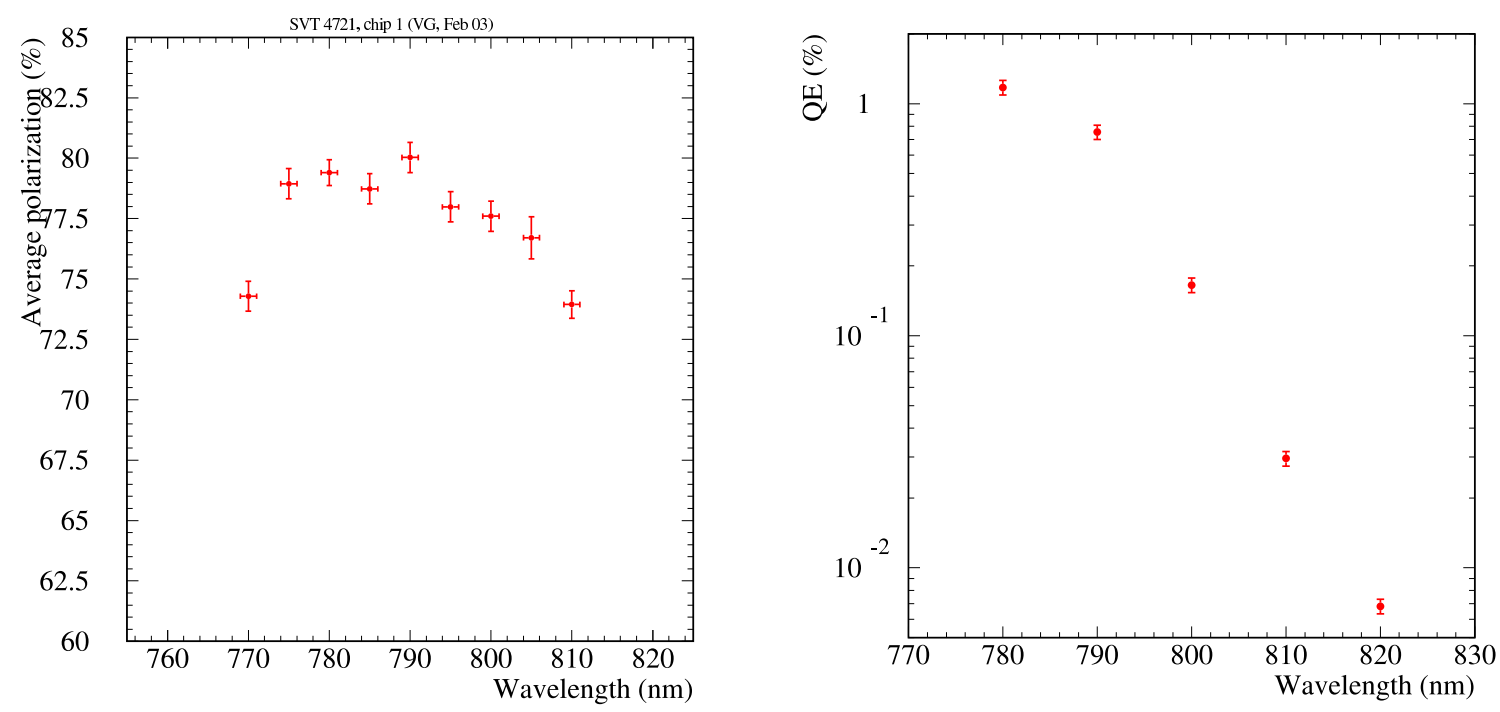

Figure 3.4. Polarization (left) and QE (right) as a function of wavelength for the superlattice photocathode. These data are provided by the JLab polarized source group [26].

the strained-layer cathode is $12 \%$ and a value of $4 \%$ is typical for the superlattice at the operating wavelength [26].

\subsubsection{Electron Guns}

JLab has two identical electron guns, Gun \#2 and Gun \# $3^{2}$, to inject electrons into the accelerator. For the 2004 HAPPEX run, Gun \#2 had a strained-layer cathode installed in it and Gun \#3 had a superlattice cathode installed. The use of the two electron guns required HAPPEX to use two configurations of the source laser table. A schematic of the source laser table used for the 2004 HAPPEX run is shown in Figure 3.5. All three experimental halls were taking data during the time that HAPPEX ran. Halls B and C used diode lasers at wavelengths of $655 \mathrm{~nm}$ and $773 \mathrm{~nm}$ respectively. Each laser is pulsed at $499 \mathrm{MHz}$ and interleaved to produce a $1497 \mathrm{MHz}$ electron beam for capture into the accelerator's RF cycle.

\footnotetext{
${ }^{2}$ Gun \#1 is the designation reserved for the thermionic gun which is no longer in use at JLab.
} 


\subsubsection{Source Optics}

Similar optics are used in each laser beam trajectory to the cathode although there are some important additions for the Hall A line because of the special requirements for a parity-violation experiment. All halls have attenuators to adjust the power of the laser light illuminating the cathode. As the QE of the cathode decreases, more laser power is necessary to maintain the current delivered to the experimental halls. Halls A and C have "tune-mode" (TMPC) optics which produce a pulsed beam structure to allow proper steering of the electron beam to the halls without causing any damage to beamline elements; Hall B uses such low current that tune-mode optics are unnecessary.

\subsubsection{Hall A Line}

The Hall A laser path includes a telescope consisting of two lenses with a focal length of $6.5 \mathrm{~mm}$ with a distance of $1.3 \mathrm{~cm}$ between them. The telescope provides the proper divergence and beam spot size at the Pockels cell and subsequent beam spot size at the cathode $(\sim 500 \mu \mathrm{m})$. Hall $\mathrm{A}$ has an intensity attenuator (IA) system which is an active feedback system consisting of a wave-plate, Pockels cell, and linear polarizer used to control the charge asymmetry of the electron beam in the hall. Hall $\mathrm{C}$ also has an IA system in their laser path because beam loading, cathode interactions, and bleedthrough cause the Hall $\mathrm{A}$ and $\mathrm{C}$ beams to interact with one another. In order to keep the charge asymmetry measured in Hall A small, it is necessary to feedback on both the Hall A and C beams.

\subsubsection{Common Optics}

The Hall A and B laser beams are combined by using a dichroic mirror and all three laser beams are combined into one beam with a polarizing beam-splitting cube. A mirror mounted on a piezo-electric actuator (PZT) is used to steer the beams along the final straight-line trajectory to the cathode. The PZT mirror can be used to make small helicity-correlated beam position corrections but was not used during the HAPPEX experiment. There is an insertable half-wave plate (IHWP) just upstream of the Pockels cell which provides a slow reversal of the laser beam helicity; and therefore, a reversal of the electron beam helicity. 


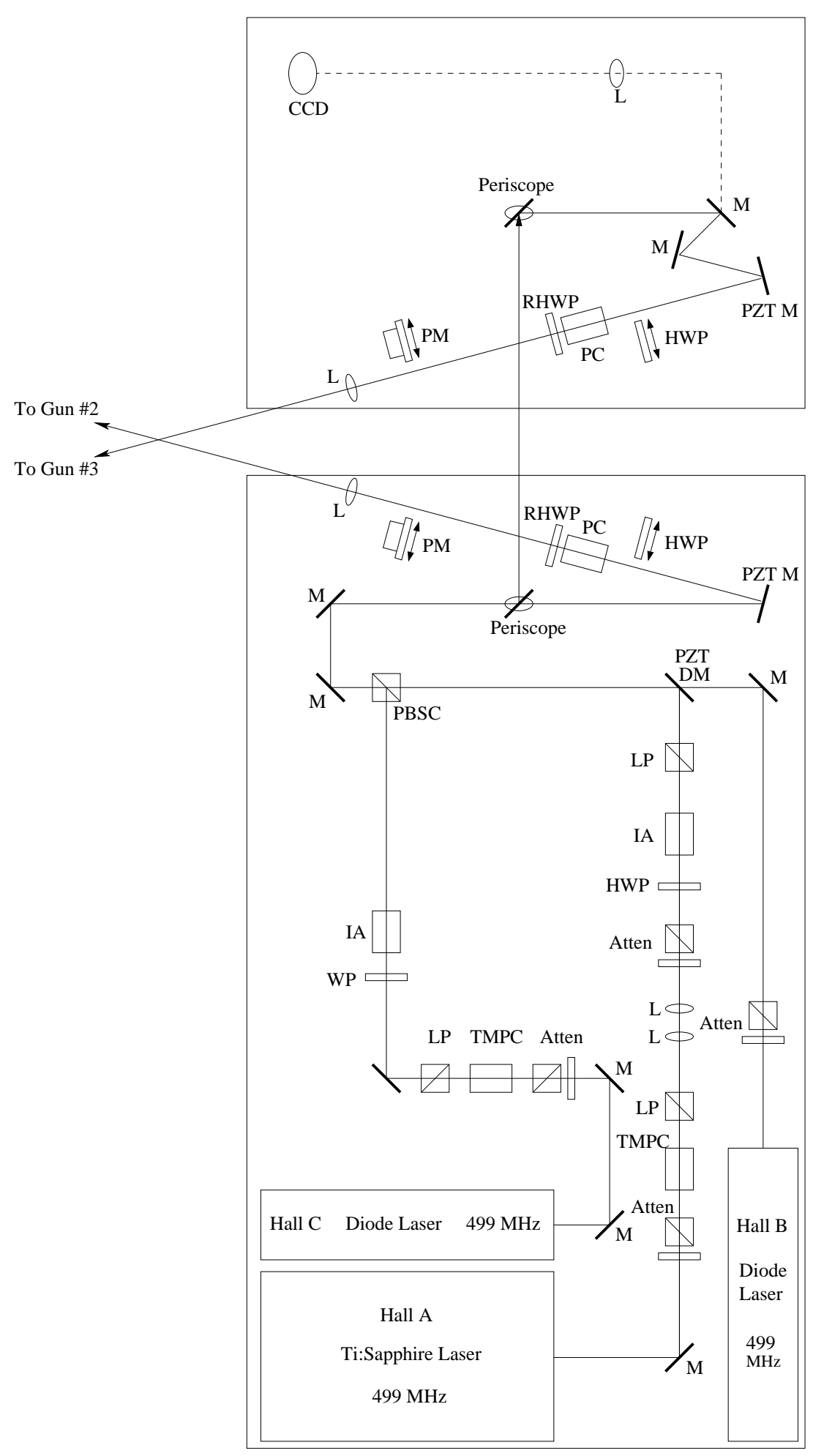

Figure 3.5. The JLab polarized source laser system as it was configured for the June/July 2004 running of HAPPEX. (M) Mirror, (DM) Dichroic Mirror, (L) Lens, (PC) Pockels Cell, (TMPC) Tune-Mode Pockels Cell, (LP) Linear Polarizer, (WP) Wave Plate, (HWP) Half-Wave Plate, (RHWP) Rotatable Half-Wave Plate, (IA) Intensity Attenuator, (Atten) Attenuators, (PBSC) Polarizing Beam-Splitting Cube, (PM) Power Meter, (CCD) Camera. 


\subsubsection{Pockels Cells}

The Pockels cell (PC) is at the heart of every parity-violation experiment because it provides the fast-reversal of the electron-beam helicity. A Pockels cell is an electro-optic device with the unique characteristic that its birefringence is linearly proportional to the electric field applied to it. A Pockels cell ${ }^{3}$ is used as a quarter-wave retarder in order to convert the linearly polarized laser light into circularly polarized laser light. A voltage of $\sim 2300 \mathrm{~V}$ and $\sim 2500 \mathrm{~V}$ for laser wavelengths of $780 \mathrm{~nm}$ and $850 \mathrm{~nm}$ respectively is applied to achieve quarter-wave retardation. The polarity of the $\mathrm{HV}$ is reversed at $30 \mathrm{~Hz}$ to reverse the helicity of the outgoing laser light. The helicity of the beam is pseudorandomly reversed at $30 \mathrm{~Hz}$ as described in Section 2.4.

The well-aligned Pockels cell produces highly circularly polarized light, but it is not perfect. After careful alignment of the PC, as described in Section A.1.5, typical values are around $99.993 \%$. The residual linear polarization of the laser beam is analyzed on the cathode and can cause large charge asymmetries and helicity-correlated position differences. For this reason, a rotatable half-wave plate is placed downstream of the PC. The RHWP rotates the residual linear polarization direction to minimize its effect on helicity-correlated beam parameters.

\subsubsection{Transport to the Cathode}

A lens is placed just upstream of the vacuum window entrance to the electron gun and is used to control the beam spot size and steer the laser beam on the cathode. When the laser spot illuminates one spot on the cathode for a long period of time, a "QE hole" can develop, and it becomes necessary to move the beam to a different spot on the cathode to maintain the beam current required for each experimental hall. The superlattice cathode lifetime was very poor during 2004, and spot moves were required almost every day when Halls A and C (both high current) were running simultaneously. The poor lifetime was due to the lack of anodization of the cathode. The cathode lifetime improved for 2005 because of new anodization techniques.

\footnotetext{
${ }^{3}$ The Pockels cell is a $\mathrm{KD}^{*} \mathrm{P}, \mathrm{KD}_{2} \mathrm{PO}_{4}$, crystal.
} 
There is a separate final laser path trajectory for each electron gun. A periscope is inserted in the beamline upstream of the PZT mirror in order to use the Gun \#3 beamline. This beamline also has a pick-off mirror to another beamline matching the throw to the cathode. This pick-off beamline terminates at a CCD camera which is used to check the beam spot size and location at the cathode. A more detailed description of the source setup and control of helicity-correlated beam parameters is discussed in Chapter 4 .

\subsection{Injector}

The electron gun is situated at an angle of $15^{\circ}$ with respect to the accelerator beamline. A solenoid is used to bend the electrons into the accelerator. A Wien filter is used to set the polarization angle of the electrons as they enter the accelerator. The angle is set such that the electron is longitudinally polarized in the experimental hall after taking into account the $g-2$ precession through the accelerator. The injector accelerates the electrons up to an energy of $45 \mathrm{MeV}$ before they enter the main accelerator.

\subsection{Beam Monitors and Beam Modulation}

Beam current monitors (BCMs) and beam position monitors (BPMs) are located throughout the accelerator and the experimental halls. Selected monitors from the injector region and the Hall A beamline are read out in the HAPPEX data stream. A schematic showing the monitors and their locations is shown in Figure 3.6. The beam monitors should be quiet and sensitive in order to precisely measure the helicity-correlated beam differences which affect the raw asymmetry measurement.

The beam monitors in the injector are used in dedicated studies to setup the polarized source optics to control helicity-correlated beam asymmetries. During normal running conditions the injector contains beam from all three experimental halls, but after the 5 $\mathrm{MeV}$ region, primarily beams from Halls $\mathrm{A}$ and $\mathrm{C}$ are present. Injector data taken during production running is used to check transmission of the beam through the injector. This is useful to ensure that the charge asymmetry and position differences measured in the hall are real and not caused by clipping of the beam on apertures in the injector. 


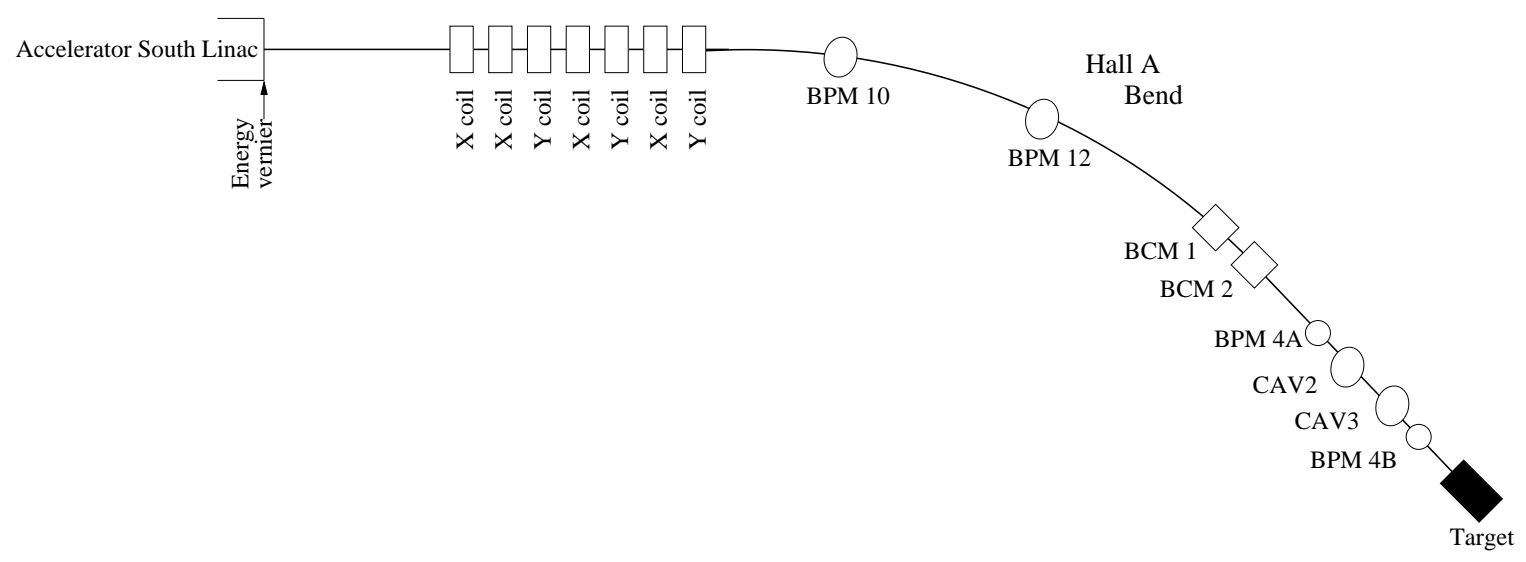

Figure 3.6. Schematic of Hall A beam monitor and beam modulation coil locations along the Hall A beamline.

\subsubsection{Beam Position Monitors}

HAPPEX uses the two BPMs located closest to the target to measure helicity-correlated position and angle differences of the beam and a BPM located in the Hall A bend to measure energy differences. BPM4A is located $\sim 6 \mathrm{~m}$ upstream of the target and BPM4B is located $\sim 1 \mathrm{~m}$ upstream of the target. Both BPMs are downstream of all optical beamline components and together the data from these two BPMs give beam position and angle information at the target. BPM12 is located in the highly dispersive region of the Hall A bend and is used to measure energy differences. The dispersive region is $4 \mathrm{~m}$ long and the dispersion is in the horizontal plane; therefore, by measuring $\Delta x$ with BPM12, we obtain a measurement of $\Delta E$.

The BPMs are wire stripline monitors which consist of 4 antennas: $X^{+}, X^{-}, Y^{+}$, and $Y^{-}$. The signal from each wire is proportional to the beam current times the distance between the beam and the antenna. All four signals for each monitor are read out and integrated in the HAPPEX DAQ. The $X$ and $Y$ antennas are positioned at $\pm 45^{\circ}$ with respect to the vertical axis $[28,29]$. The measurement of beam position can be determined independently of the beam current for each pair of wires:

$$
x^{\prime}=\frac{X^{+}-X^{-}}{X^{+}+X^{-}}, \quad y^{\prime}=\frac{Y^{+}-Y^{-}}{Y^{+}+Y^{-}} .
$$


The $x$ and $y$ beam positions are then determined by performing a rotation on the $x^{\prime}$ and $y^{\prime}$ values. The wires of the BPMs in the $100 \mathrm{keV}$ region of the injector are positioned along the horizontal and vertical axes; therefore, no rotation of the calculated positions is necessary.

\subsubsection{Beam Current Monitors}

There are three current monitors in Hall A located $\sim 25 \mathrm{~m}$ upstream of the target. Two of the current monitors, "BCM1" and "BCM2" are RF resonant cavities while the third is an Unser monitor. The RF cavities are used to measure the beam current during production running. The cavities are tuned to the frequency of the accelerator such that they output a voltage signal proportional to the beam current. The HAPPEX DAQ reads out and integrates three different amplifications of the BCM2 signal: a $1 \times, 3 \times$, and $10 \times$ and the $1 \times$ of BCM1. In the data stream, the amplifications of BCM2 are designated as "BCM2", "BCM3", and "BCM4" respectively. The $1 \times$ signals from the BCMs provide a linear response to the beam current above $\sim 10 \mu \mathrm{A}$ and from $\sim 2 \mu \mathrm{A}$ for the amplified signals. The Unser monitor provides an absolute reference for the beam current and is used for calibration of the cavity signals.

\subsubsection{Beam Cavity Monitors}

New beam cavity monitors were built by JLab to accommodate the position and intensity measurement resolution needs of the parity program. Three cavity triplets for high precision $(1 \mu \mathrm{m})$ and high bandwidth $(100 \mathrm{kHz})$ position measurements were built for Hall A [30]. Each cavity triplet consists of two orthogonal TM110-mode cavities to measure $X$ and $Y$ positions and one TM010-mode cavity to measure current $(I)$. A digital receiver samples the signals from each cavity. The signals are digitally processed and the beam intensity and $X-Y$ positions are calculated with the previously mentioned resolution and bandwidth.

Two of the cavity triplets were installed in Hall A: CAV2 is located $1 \mathrm{~m}$ downstream of BPM4A, and CAV3 is located $1 \mathrm{~m}$ upstream of BPM4B such that the two triplets are 3 $\mathrm{m}$ apart (see Figure 3.6 for a layout schematic). The third cavity triplet (CAV1) was used as a test cavity for diagnostic purposes; therefore, it was placed outside of Hall A along the beamline in the beam switchyard. Because the cavity signal size is so much larger than 
the present stripline BPMs, they should provide higher resolution measurements of beam position differences necessary for future parity experiments. The cavities were commissioned during the 2005 HAPPEX run [31].

\subsubsection{Beam Modulation}

Beam modulation, also referred to as "dithering," is a technique used by the experiment to measure the change in the detector flux for a known change in position and energy on the target. There are seven modulation coils (four $X$ coils and three $Y$ coils) and one energy vernier used to make the small changes in the beam position $(\leq 300 \mu \mathrm{m})$ and energy $(\leq 300$ $\mathrm{keV}$ ) at the target. The energy vernier is the last cavity in the south linac of the accelerator and affects all three experimental halls. Special effort was made to ensure that the changes in energy had a minimal effect on the data taking in Halls B and C. The modulation coils are located in the Hall A line upstream of the hall as shown in Figure 3.6.

\subsection{Target and Raster}

HAPPEX requires a dense, high-power target in order to achieve the desired luminosity. The experiment uses a $20 \mathrm{~cm}$ long, unpolarized, liquid hydrogen $\left(\mathrm{LH}_{2}\right)$ cryotarget operated at a temperature of $19 \mathrm{~K}$ and pressure of $\sim 25$ psia for a target density of $0.072 \mathrm{~g} / \mathrm{cm}^{3}$. The "racetrack" target cell, shown in Figure 3.7, was used for the first time for HAPPEX. The target cell is made of $\mathrm{Al}$ and is $2 \mathrm{~cm}$ in diameter. The sidewalls of the cell are $137 \mu \mathrm{m}$ thick with entrance and exit windows $178 \mu \mathrm{m}$ and $71 \mu \mathrm{m}$ thick respectively. The target cell is mounted on the Hall A target ladder assembly inside an evacuated $\left(\sim 10^{-6}\right.$ Torr $)$ scattering chamber.

The Hall A target ladder assembly consists of three cryogenic loops and several solid targets used for optics and background measurements. Installed on loop 1 was a $20 \mathrm{~cm}$ racetrack cell for a dense helium gas target. On loop 2 the $20 \mathrm{~cm}$ racetrack cell of $\mathrm{LH}_{2}$ used for production running was installed. The $15 \mathrm{~cm} \mathrm{LH}_{2}$ target installed on loop 3 was the "beer can" style cell used by the first HAPPEX experiments in 1998 and 1999. This cell was installed as a backup for the $20 \mathrm{~cm} \mathrm{LH}_{2}$ racetrack cell. Carbon, aluminum, and empty targets were used for the optics and background measurements. $\mathrm{A}_{2} \mathrm{O}$ cell, also called the 


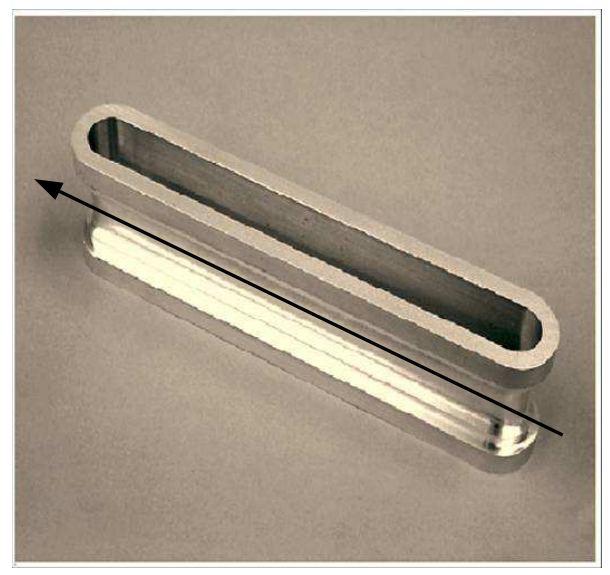

Figure 3.7. The racetrack target cell used for HAPPEX before installation on the target ladder.

waterfall target, was installed for a dedicated measurement of the scattering angle necessary to determine $Q^{2}$ at the $1 \%$ level.

The heat load on the $\mathrm{LH}_{2}$ target from a $100 \mu \mathrm{A}$ beam is $\sim 500 \mathrm{~W}$. There is some additional heat load on the target from the circulating fan and small heater used to stabilize the target temperature. At the design luminosity for HAPPEX, $100 \mu \mathrm{A}$ on a $20 \mathrm{~cm} \mathrm{LH}_{2}$ target, the demand for cooling is very high and was supplied by the $4 \mathrm{~K}$ line of the Central Helium Liquifier (CHL). The flow available to the target is controlled using Joule-Thompson (JT) valves. A low-power heater is used to regulate the temperature of the target while a high-power heater is used to maintain the heat load on the target when the beam is off. The heater provides a heat load up to $\sim 600 \mathrm{~W}$. The target temperature is controlled using a computer controlled feedback system.

Luminosity variations caused by target density fluctuations are difficult to measure. Density fluctuations over long time periods are not a concern because of the rapid helicity reversal. Small, random variations due to target boiling are a concern because they can cause nonstatistical scaling of the asymmetry width. At the design beam current of 100 $\mu \mathrm{A}$, the beam deposits $\sim 500 \mathrm{~W}$ of power onto the $\mathrm{LH}_{2}$ target, quickly by using a flow of hydrogen transverse to the beam direction. and the beam spot size is $\sim 100 \mu \mathrm{m}$ in diameter which can cause local heating of the target even at low currents. 


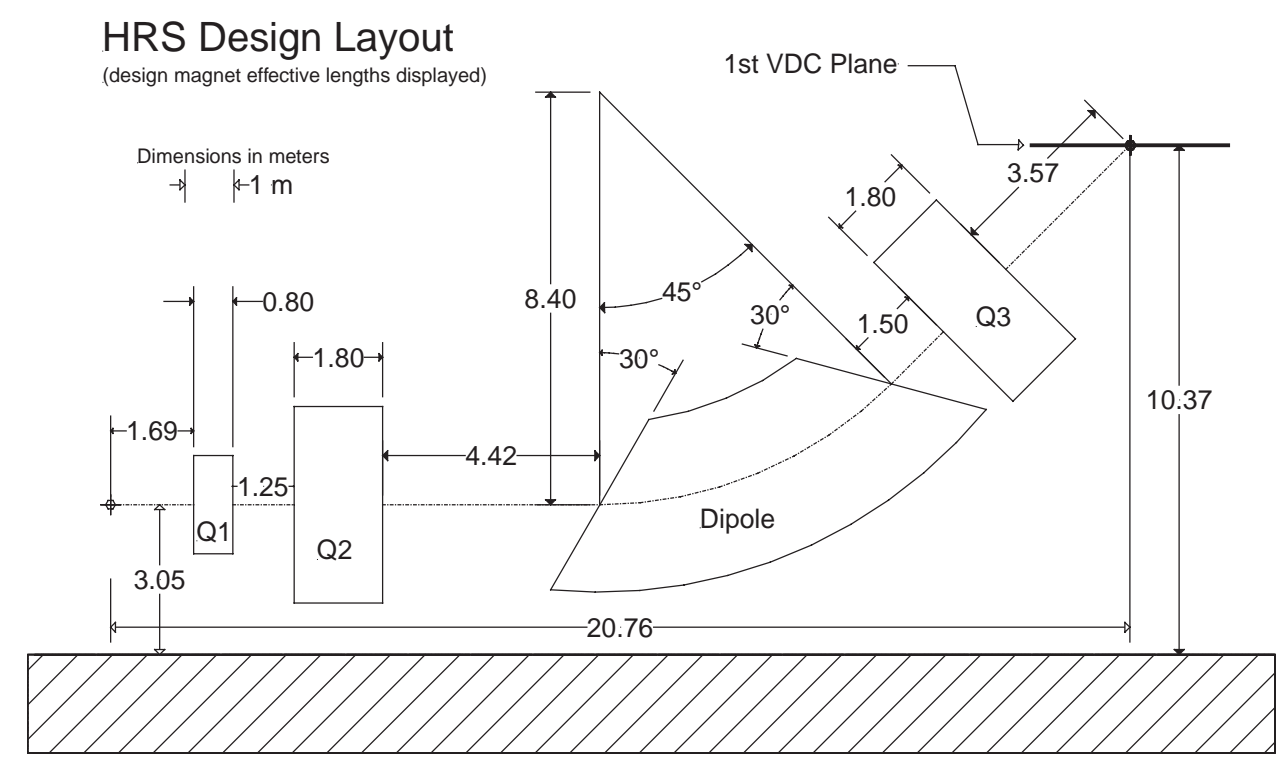

Figure 3.8. A schematic of the HRS design layout also showing the first VDC plane.

Two simultaneous methods are used to control beam heating of the target. Heat is quickly dissipated by using a flow of hydrogen transverse to the beam direction, and the heat is distributed by rastering the beam over an area $4.8 \mathrm{~mm} \times 4.8 \mathrm{~mm}$ incident on the target (in 2005 , the raster was $6.0 \mathrm{~mm} \times 3.0 \mathrm{~mm}$ ). The raster consists of two dipole magnets, one vertical and one horizontal, located $23 \mathrm{~m}$ upstream of the target. The raster is driven by a $25 \mathrm{kHz}$ triangular waveform such that the beam is uniformly distributed over a rectangular area on the target [32]. Estimates of the density fluctuations made by scanning parameters such as raster size and cryogen-loop fan speed suggest that density fluctuations are controlled at the $100 \mathrm{ppm}$ level and are not a significant problem.

\subsection{Hall A Spectrometers and Septum Magnets}

The Hall A High Resolution Spectrometers (HRS) consist of two identical magnetic spectrometers. The HRS is a QQDQ superconducting magnet design: two quadrupoles, a vertically bending dipole $\left(45^{\circ}\right)$, and a third quadrupole shown in Figure 3.8. The dipole provides high momentum resolution $\left(10^{-4}\right)$ because of the $12 \mathrm{~m}$ dispersion, and the quadrupoles provide focusing. Because of the size of the apparatus, the minimum scattering angle of the 


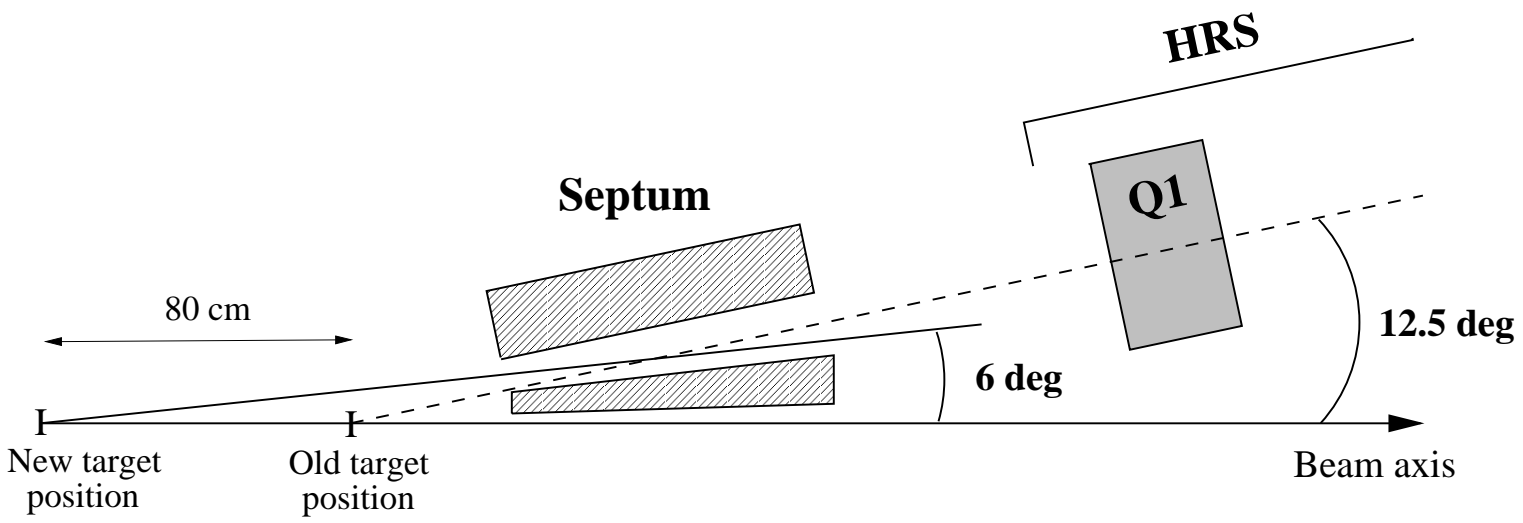

Figure 3.9. A diagram showing the HRS setup with the septum installed.

HRS is $12.5^{\circ}$ and both HRS are placed symmetrically about the beamline at this angle. The HRS on each side of the beamline (looking along the beam direction) is called the Left HRS (LHRS) or left arm and the Right HRS (RHRS) or right arm. The HAPPEX kinematics require a very forward scattering angle $\left(6^{\circ}\right)$; therefore the experiment uses superconducting septum magnets upstream of the HRS in order to bend the $6^{\circ}$ scattered electrons into the opening aperture of the HRS. With the septum magnets installed, the target location is moved $80 \mathrm{~cm}$ upstream in order to stay on the optical axis of the HRS. A schematic showing the HRS with the septum magnets installed is in Figure 3.9. The symmetry of the apparatus doubles the counting statistics and provides a means to cancel position differences. Details of the HRS, septum apparatus, and the optical transport matrices can be found in $[33,34,35]$.

\subsubsection{High Resolution Spectrometers}

The HRS magnetic fields are set using the EPICS (Experimental Physics and Industrial Control System $)^{4}$ interface such that the central momentum of the spectrometer focuses the elastically scattered electrons onto the focal plane into the detector. The dipole fields are measured and monitored by NMR probes while the quadrupole fields are monitored by Hall probes. The fields in the quadrupoles are set based on their current settings because

\footnotetext{
${ }^{4}$ EPICS documentation, http://www.aps.anl.gov/epics
} 


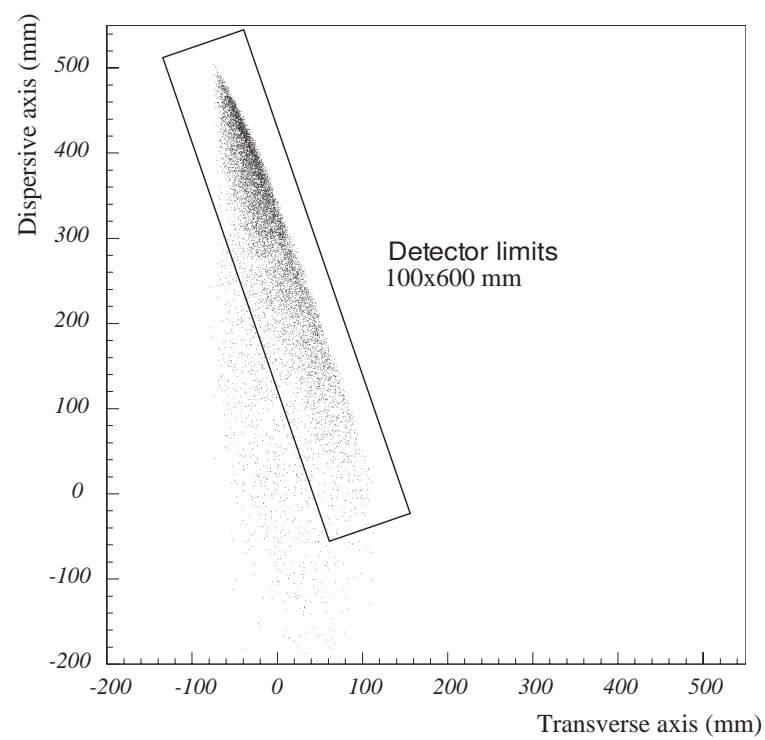

Figure 3.10. The simulated elastic peak focused onto the focal plane by the Hall A HRS and the detector footprint are shown.

the Hall probes are not stable or reliable over long time periods [33]. The HRS momentum resolution provides a clean separation of elastic events from inelastic background ensuring the integration technique is reliable. The focused elastic peak in the focal plane and the detector footprint over the peak is shown in Figure 3.10.

\subsubsection{Septum Magnets}

The septum magnet field is controlled by setting the current via the EPICS interface. The coolant flow through the right septum was limited due to a blockage in the helium coolant circuit (from the time of manufacturing) and a leaky control valve [36]. The septum current was actually set at a value $+10 \%$ of the central momentum setting because it provided better cooling without affecting the spectrometer acceptance. The septum magnets also had problems with heating due to low-energy Møller electrons impinging on the bore of the magnets. Some special tungsten collimators were built to help reduce the heating as well as some lead shielding placed just upstream of each septum. The beam-induced heating was still a significant problem because of the thick target used and limited the beam current of the experiment to $38 \mu \mathrm{A}$ in 2004 . The beam current was only limited to $58 \mu \mathrm{A}$ in 2005 


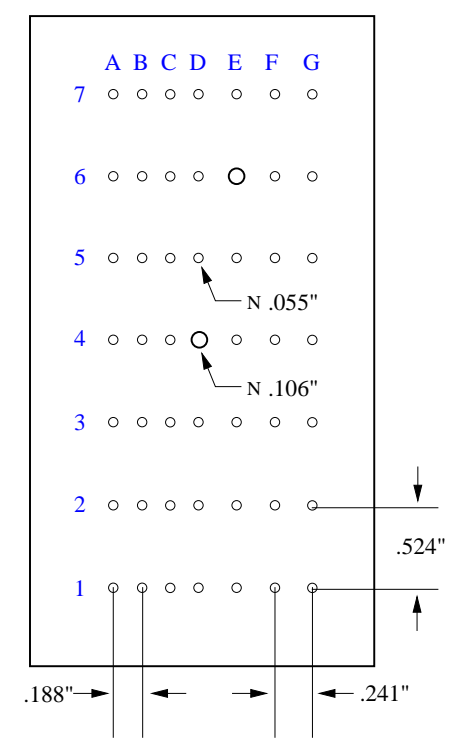

Figure 3.11. Insertable sieve-slit hole-pattern used for optimization of the spectrometer transfer matrices.

because septum heating was reduced by the installation of a sweeper magnet that swept low-energy Møller electrons back toward the beampipe and away from the septum bore [37].

\subsubsection{Hall A Standard Detector Package}

The Hall A standard detector package including Vertical Drift Chambers (VDCs) and scintillators is used in counting-mode for measuring the HRS optics, $Q^{2}$, scattering angle, and backgrounds. The scintillators are used for triggering, and the VDCs are used for reconstruction of particle trajectories. An insertable sieve slit (Figure 3.11) is located in a transfer box connecting the scattering chamber to the septum magnet and is rotated into place for optics studies.

The detectors are located in a shield hut which protects the detectors from background radiation. For details of the shield hut properties, see [33]. Additional shielding is also provided by a Line-of-Sight Block which is a two-meter thick concrete block located on top of the first two quadrupoles of the HRS. The DAQ electronics are also placed in the shield hut for protection from radiation damage. 


\subsection{HAPPEX Focal-Plane Detectors}

The HAPPEX focal-plane detectors are total absorption Čerenkov-shower calorimeters. The Cerenkov light is collected by a photomultiplier tube (PMT), and the signal is integrated over each helicity window to allow a high counting rate $(\sim 60 \mathrm{MHz})$ without deadtime. The design of the detectors was modelled after the first-generation HAPPEX detectors using a

sandwich design for high energy resolution [19]. There were some significant changes to the original design because of the very forward-angle kinematics of this experiment discussed below.

Because the scattering rate is so high at such forward angles, the radiation dose the detector receives is $\sim 1 \mathrm{MRad}$; therefore, radiation-hard materials were chosen for the construction of the detectors. A sandwich design made up of 5 alternating layers of fused quartz (Spectrosil 2000) and brass were chosen to obtain good energy resolution $(17 \% \sigma)$. The energy resolution can broaden the statistical width of the asymmetry by

$$
\sigma(A)=\frac{1}{\sqrt{N}} \sqrt{1+\left(\frac{\Delta E}{\langle E\rangle}\right)^{2}} .
$$

The achieved energy resolution contributes less than $2 \%$ to the asymmetry width. The detector covers an area $10 \mathrm{~cm}$ wide and $60 \mathrm{~cm}$ long as shown in Figure 3.10. The detector dimensions were chosen to entirely overlap the elastic stripe and to ensure the electromagnetic shower is well contained in the detector while excluding inelastic events.

The decision to use an "L"-shaped segmented detector design, shown in Figure 3.12, is another change from the original HAPPEX detector. Each segment is equal in length. The fused quartz in each segment is $1 \mathrm{~cm} \times 10 \mathrm{~cm} \times 30 \mathrm{~cm}$, and the brass layers are 1.5 $\mathrm{cm} \times 10 \mathrm{~cm} \times 30 \mathrm{~cm}$. An additional brass layer at the detector entrance is $4.5 \mathrm{~cm}$ thick to center the shower maximum in the detector. The segmentation allows the measurement of the asymmetry at two near $Q^{2}$ values and provides a highly sensitive test of helicitycorrelated beam-asymmetry corrections. The drawback of the segmentation is the increased sensitivity to edge effects. Edge effects contribute at most a $10 \%$ increase to the desired energy resolution; but summing the two detector signals before calculating the asymmetry makes crosstalk between the two segments negligible. 


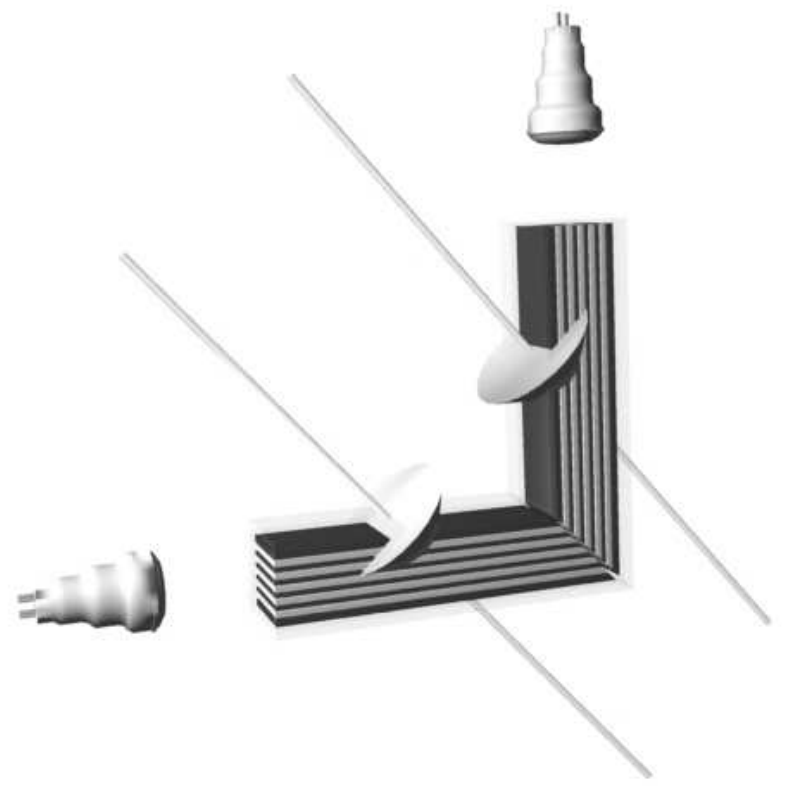

Figure 3.12. A drawing of the focal-plane detector with the elastically scattered electron flux coming up from the right. The cone of Cerenkov light from the electromagnetic shower is imagined.

The scattered electrons reach the focal plane at $45^{\circ}$ with respect to the horizontal plane and the C̆erenkov cone opens at $47.2^{\circ}$. The detector is aligned along the Cerenkov cone edge for maximum light collection. The light is transmitted along the length of the detector by internal reflections in the quartz. There is a $20 \mathrm{~cm}$ metallic light guide connecting the detector to the PMT. The light is collected by 5" diameter BURLE 8854 PMTs. Additional detector details can be found in [38].

One "L"-shaped detector sits $3.5 \mathrm{~cm}$ above the focal plane in each HRS. Det1 and Det2 are the detector segments on the left, and Det3 and Det4 are on the right. Det1 and Det3 are the low $Q^{2}$ detectors and Det2 and Det 4 are the high $Q^{2}$ detectors. After alignment of the detectors in each HRS arm, the detector segments had different acceptance bites such that (in 2004) Det2 (65\%) and Det3 (75\%) were the high-rate detectors and Det1 (35\%) and Det4 (25\%) were the low-rate detectors. The linearity of each PMT was tested during the experiment, and each PMT was linear to better than 1\%. Additional linearity studies conducted during June 2005, using LEDs to mimic beam conditions, confirm these results $[39]$. 


\subsection{DAQ}

The HAPPEX data acquisition (DAQ) system is unique from the standard Hall A DAQ because it integrates and digitizes the signals from the detectors and beam monitors. The DAQ is built of high resolution (16-bit) analog-to-digital converters (ADCs) and ad-hoc ADCs constructed of voltage-to-frequency converters coupled with gated scalers (V2Fs). The DAQ is triggered at $30 \mathrm{~Hz}$ synchronized to the helicity signal. Integrating over $33 \mathrm{~ms}$, the $2 \mathrm{MHz}$ V2Fs also provide 16-bit resolution.

The DAQ is similar to what was used during the first-generation HAPPEX [19]. The DAQ is triggered by the $30 \mathrm{~Hz}$ helicity signal, but the integration only begins at the end of the $500 \mu$ s pulse marking the end of the helicity instability due to the HV change on the PC. We integrate for a total of $32.5 \mathrm{~ms}$ to ensure that we end the integration before the helicity reversal for the next window occurs. There are four synchronized VME crates containing all of the integrating electronics: injector, counting house, LHRS, and RHRS crates. Because of possible electronics pickup from the helicity signal, the real-time helicity signal is not sent to any of the DAQ crates. In fact, the only helicity information sent to the DAQ was a helicity signal delayed by eight helicity windows, and it was only sent to the injector and counting-house crates during the 2005 data-taking period.

The ADCs can be used in voltage or current integrating mode by a simple hardware change to the board. The detectors are read out by current integrating ADCs and the beam monitors are read out by voltage integrating ADCs or V2Fs. Some channels of the ADCs are connected to batteries in order to make measurements of helicity pickup in the electronics. Details of the ADC and DAQ electronics can be found in [19, 21, 22].

The DAQ is controlled by a VME computer using the JLab DAQ software CODA. The VME controller handles communication between the DAQ and other system parameters. In particular, the counting-house crate is used to control the intensity feedback, HV on the PC, RHWP scans, and beam modulation. The DAQ also records many EPICS variables (which are only reported on a $4 \mathrm{~s}$ timescale) into the data stream such as the status of the polarized source optics, accelerator systems, target and raster parameters, septum and HRS magnet properties, and detector HV. 


\subsection{Polarimetry}

The theoretical parity-violating asymmetry is defined for a beam that is $100 \%$ polarized. Because the experimental beam is not fully polarized, the experimental physics asymmetry is

$$
A_{\text {exp }}=\frac{A_{\text {corr }}}{P_{b}}
$$

where $A_{\text {corr }}$ is the raw asymmetry corrected for beam asymmetries and $P_{b}$ is the electron beam polarization. The beam polarization is defined as

$$
P_{b}=\frac{N_{\uparrow}-N_{\downarrow}}{N_{\uparrow}+N_{\downarrow}}
$$

where $N_{\uparrow}\left(N_{\downarrow}\right)$ is the number of electrons aligned parallel (antiparallel) to the beam direction. The longitudinal beam polarization for HAPPEX was measured by the Møller and Compton polarimeters in Hall A.

\subsubsection{Møller Polarimetry}

The Hall A Møller polarimeter is described in detail in [33]. Møller polarimetry measures the asymmetry in electron-electron scattering where the cross section for the scattering process depends on the beam and target polarizations as well as the analyzing power of Møller scattering. The target foil is polarized along its plane and is oriented at an angle of $\pm 20^{\circ}$ with respect to the beam in the horizontal plane. The target angle makes the Møller measurement sensitive to both longitudinal and transverse beam polarization. The polarization is determined by averaging the measurements from the two target foils which cancels the transverse contributions and helps cancel helicity-correlated beam asymmetries. The Møller measurement is inherently invasive to data-taking because it has to be conducted at very low beam currents. A Møller measurement was taken every couple of weeks during production running. The final systematic error achieved by the Møller is $\sim 3 \%$ and is due mainly to the uncertainty in the target polarization. 


\subsubsection{Compton Polarimetry}

The Hall A Compton polarimeter is described in detail in [40]. Compton polarimetry measures the asymmetry in scattering polarized electrons off of circularly polarized photons defined as

$$
A_{\exp }=\frac{N_{+}-N_{-}}{N_{+}+N_{-}}
$$

where $N_{+}\left(N_{-}\right)$is the Compton counting rate for right (left) helicity electrons normalized to the beam intensity. The beam polarization is

$$
P_{b}=\frac{A_{\mathrm{exp}}}{P_{\gamma} A_{\mathrm{th}}}
$$

where $P_{\gamma}$ is the photon polarization $(>99 \%)$ and $A_{\text {th }}$ is the Compton analyzing power. The Compton interaction point is located in the center of a magnetic chicane in order to separate the scattered electrons and photons from the primary beam. The Compton uses a Fabry-Pérot cavity to increase the photon density at the interaction point. The cross section for the Compton process is so small that the primary beam is delivered to the experimental target unchanged; therefore, the Compton polarization measurement can be conducted simultaneously to production data-taking. The Compton measurement is conducted for right and left circularly polarized light in order to reduce helicity-correlated beam asymmetry effects. The relative systematic error achieved by the Compton is $1-1.5 \%$.

\subsection{0 $Q^{2}$ Profile Scanners}

Because $Q^{2}$ varies over the spectrometer acceptance, the average $Q^{2}$ must be known with good accuracy. This measurement uses the VDCs which can only operate at rates $<1 \%$ of the full HAPPEX luminosity; therefore, extremely low beam currents are used for this measurement. A profile scanner for each HRS arm was built by UMass to operate at production beam currents in order to check that the measured $Q^{2}$ distribution at low current matches the distribution during production running conditions. The scanner was

used in integrating mode to measure the $Q^{2}$ distribution approximately once per day during production running. 


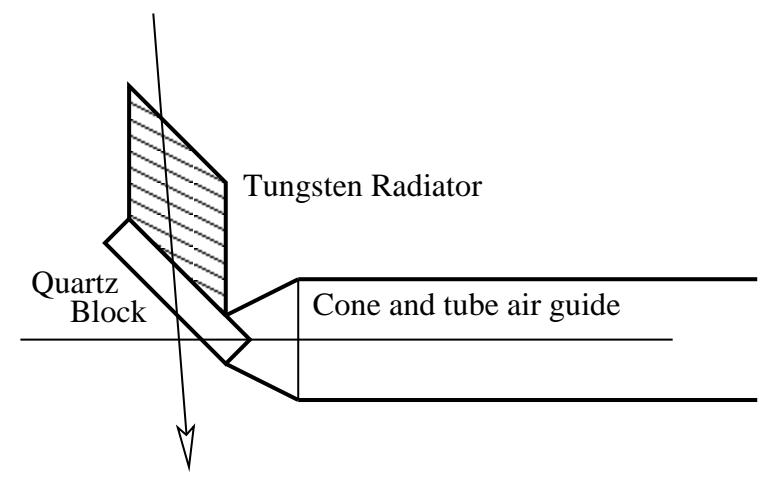

Figure 3.13. A schematic of the $Q^{2}$ profile scanner detector.

The scanner design resembles that used for SLAC Experiment E158 [41]. The detector is made of a $20 \mathrm{~mm} \times 5 \mathrm{~mm} \times 5 \mathrm{~mm}$ quartz block inclined at $45^{\circ}$ to the electron beam. In front of the quartz is a rhombic tungsten radiator $15 \mathrm{~mm}$ thick in the beam direction. The light from the quartz is directed down a $50 \mathrm{~cm}$ air light guide lined with Alzak, for high reflectivity, to a single high-gain PMT (1 inch diameter Photonics XP2982). A schematic of the detector is shown in Figure 3.13 .

Each scanner is placed on two Parker-Daedal 406XR drives aligned perpendicularly with one on top of the other such that the focal plane can be scanned in both the transverse $(y)$ and dispersive $(x)$ directions. The drive motion is controlled remotely by a LabView Flexmotion application which runs on a computer in the Hall A counting house and connects to a four-channel National Instruments FW-7344 motion controller in the detector hut via an IEEE-1394 (firewire) bus. Each of the linear drives was fit with a UniMeasure LX-PA linear potentiometer $(0-10 \mathrm{~V})$ for real-time position readout in the DAQ which correlates to the rotary encoder information that provides high-accuracy position readout in the LabView motion application. Calibration of the encoders and potentiometer readout between the DAQ and LabView output was done before production running began and remained constant throughout the experiment. The scans provide a map of the flux rate over the focal plane, and we verify that this distribution remains constant throughout the experiment. 


\subsection{Luminosity Monitor}

The luminosity monitor is mainly used to measure effects due to target density fluctuations. The luminosity monitor is located $7 \mathrm{~m}$ downstream of the target and consists of eight detectors symmetrically placed about the beampipe but staggered slightly along the beam direction in order to reduce stress on the beampipe. The luminosity monitor is sensitive to scattering angles ranging from $0.5^{\circ}$ to $0.8^{\circ}$.

Each "lumi" detector consists of a rectangular piece of fused quartz (Spectrosil 2000) where light from electrons undergoing Cerenkov radiation travels through the quartz and an aluminum-walled cylindrical light guide to an R7723 PMT. The flow of nitrogen around the PMT is necessary to protect the PMT cathode from degradation due to helium gas present in Hall A [42]. The lumi signal is integrated in the HAPPEX DAQ, and each detector has a small statistical width due to the high scattering rates at such a small scattering angle. Target density fluctuations were determined to be at the level of $100 \mathrm{ppm}$ and only contributed $2 \%$ to the asymmetry's statistical width. 


\section{CHAPTER 4 POLARIZED BEAM AT JEFFERSON LAB}

The HAPPEX source optical system is shown in Figure 3.5 and a description of the various optics is described in Section 3.2. The JLab polarized source group dedicated the laser room test stand for the HAPPEX experiment's use to study helicity-correlated beam asymmetries in the effort to better understand their sources and how to control them. The rest of this chapter discusses the results of that effort. This work was essential to sufficiently minimize the systematic errors on the parity-violating asymmetry due to helicity-correlated beam asymmetries.

\subsection{Sources of Helicity-Correlated Beam Asymmetries}

Helicity-correlated beam asymmetries can be the dominant systematic error in a parityviolation experiment if no effort is made to control and correct for them. The dominant source of helicity-correlated beam asymmetries is from a difference in the number of electrons incident on the target for each helicity state which causes a charge asymmetry, $A_{Q}$. We must also worry about significant effects which arise from helicity-correlated differences in position and angle on target. Significant effort has been made to understand the sources of position differences in order to control them at a satisfactory level.

As the precision of parity-violation experiments increases, it will also be necessary to worry about non-linear effects such as beam spot-size asymmetries which are much harder to measure and control.

\subsubsection{The PITA Effect}

The most significant contribution to helicity-correlated beam asymmetries is imperfect circular polarization produced by the Pockels cell. When the helicity of the beam is reversed, the major axis of the polarization ellipse is rotated by $90^{\circ}$. Because optical elements tend 
to transmit one polarization state better than another, an analyzing power of the optical system arises called the PITA effect where PITA means "polarization induced transport asymmetry" [43]. The formalism of the PITA effect is described in detail in [43] and for a more complex optical system in [44].

As described in Section 3.2.2.3, the Pockels cell is an electro-optic device whose birefringence is linearly proportional to the voltage applied to it. For our purposes, high voltage is applied to the Pockels cell in order to create a $\pm \pi / 2$ phase shift on the laser beam which converts linearly polarized laser light to right or left circularly polarized light. The phase shift produced by the Pockels cell is parameterized by

$$
\delta_{R}=-\left(\frac{\pi}{2}+\alpha\right)-\Delta \quad \delta_{L}=+\left(\frac{\pi}{2}+\alpha\right)-\Delta
$$

where $\delta_{R}\left(\delta_{L}\right)$ is the phase shift for producing right (left) circular polarization and $\alpha$ and $\Delta$ represent symmetric and antisymmetric offsets respectively from perfect circular polarization. The $\alpha$ phase is called symmetric because a non-zero value causes residual linear polarization of the same sign for both helicity states, and the $\Delta$ phase is called antisymmetric because a non-zero value creates oppositely signed residual linear polarization in the two helicity states as illustrated in Figure 4.1. In effect, the $\Delta$ phase gives a larger phase shift to one helicity state and decreases the phase shift for the other. In the asymmetry measurement, the $\alpha$ phase effect cancels, and we are only sensitive to the $\Delta$-phase effects.

The photocathode provides the dominant analyzing power in our optical system due to the strain induced on the photocathode to produce high polarization. We define the cathode with different QE along orthogonal axes $x^{\prime}$ and $y^{\prime}$ where the $x^{\prime}$ axis makes an angle $\theta$ with the horizontal. If linear polarized light is aligned along the $x^{\prime}\left(y^{\prime}\right)$ axis, then it will have a transmission coefficient of $T_{x^{\prime}}\left(T_{y^{\prime}}\right)$. We then define the quantities $\epsilon=T_{x^{\prime}}-T_{y^{\prime}}$ and $T=\left(T_{x^{\prime}}+T_{y^{\prime}}\right) / 2$. The charge asymmetry is calculated to be

$$
A_{Q}=\frac{I_{R}-I_{L}}{I_{R}+I_{L}}=-\frac{\epsilon}{T}\left(\Delta-\Delta^{0}\right) \cos 2 \theta
$$

where $I_{R}\left(I_{L}\right)$ is the electron beam intensity for a Pockels cell phase shift $\delta_{R}\left(\delta_{L}\right)$ and $\Delta^{0}$ is an offset included to account for residual birefringence in the Pockels cell and the 


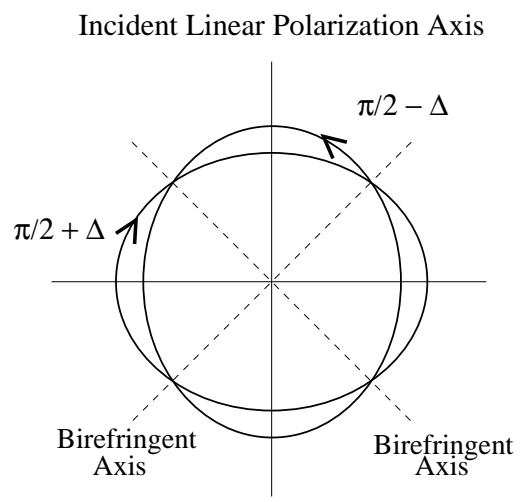

Figure 4.1. Polarization ellipses for a non-zero $\Delta$ phase $(\alpha=0)$ where the birefringent axes are $45^{\circ}$ to the horizontal.

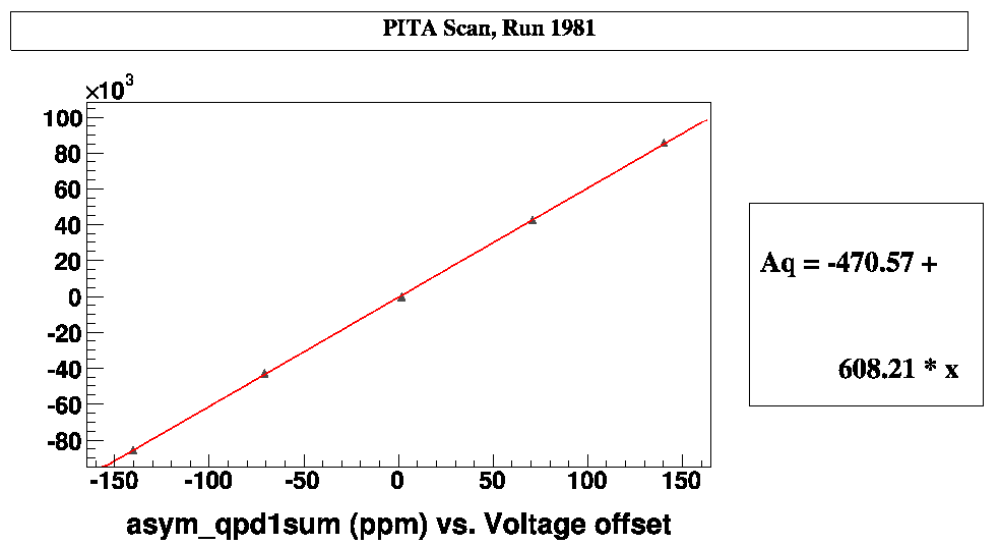

Figure 4.2. PITA slope, $A_{Q}(\mathrm{ppm})$ versus voltage offset $(\mathrm{V})$, measured using an LP analyzer downstream of the PC and a maximum $V_{\Delta}= \pm 140 \mathrm{~V}$.

downstream optics. This result conveniently shows that $A_{Q}$ is linearly dependent on $\Delta$ only; therefore, we can control the size of $A_{Q}$ by controlling the $\Delta$ phase.

By changing the voltage applied to the Pockels cell by $V_{\Delta}=\Delta \cdot \frac{V_{\lambda / 2}}{\pi}$ where $V_{\Delta}$ is the voltage required to induce a phase shift of $\Delta$ and $V_{\lambda / 2}$ is the voltage required for half-wave retardation, we can measure the PITA slope defined as $\frac{\epsilon}{T} \cos 2 \theta$. The PITA slope gives us a measure of our sensitivity to the system's analyzing power. A typical PITA slope of $\sim 600 \mathrm{ppm} / \mathrm{V}$ as shown in Figure 4.2 is achieved when using a linear polarizer (LP) as an analyzer. Typical PITA slopes off of the cathode are between 5-15 ppm/V. 


\subsubsection{Charge Asymmetry Structure}

We identified an effect which couples to the analyzing power and causes a multipeak structure of the charge asymmetry. When the high voltage is applied to the Pockels cell, there is a time constant associated with the PC retardation. In each helicity window, there is a time dependence of the $\mathrm{PC}$ retardation which is also dependent on the preceding helicity windows.

Early in our studies we understood that there was a time constant associated with the PC "remembering" the helicity from the window immediately preceding a pair, but further investigation revealed that the PC retardation remembers the helicity over a long time period. The green line in Figure 4.3 shows the helicity pattern of the HV applied to the PC and the black curve shows the transmission through an LP crossed with the incoming polarization, such that transmission increases as the PC retardation approaches $\pi / 2$. The continual change in transmission over the helicity window affects the average intensity of the laser beam over a helicity window (and therefore, the charge on target). For this reason, the charge asymmetry also has a time-dependent structure shown in Figure 4.4. This was measured by running our DAQ in "oversample" mode so that it could make several measurements during each helicity window. The reason there are four different curves is explained below.

Figure 4.3 clearly shows that when the PC sits in a helicity state for two consecutive windows, there is a greater change in transmission when the helicity reverses than if there are three windows of alternating helicity states. As a result, the charge asymmetry develops a multipeak structure when an analyzing power is present.

The multipeak structure is dependent on the helicity sequence pattern, and the separation between peaks is proportional to the PITA slope and the residual birefringence of the PC. Figure 4.5 shows the $A_{Q}$ multipeak structure for three possible helicity sequence patterns. The pair-toggle (black) is a helicity pattern that simply toggles $R L R L R L \ldots$ This pattern produces only one $A_{Q}$ peak because the $\mathrm{HV}$ change on the $\mathrm{PC}$ is the same for all asymmetry pairs. The quad-random helicity pattern pseudorandomly chooses the helicity state for every fourth window such that there are two possibilities for a set of four windows, $R L L R$ or $L R R L$. This helicity pattern provides four distinct possibilities for the HV switch 


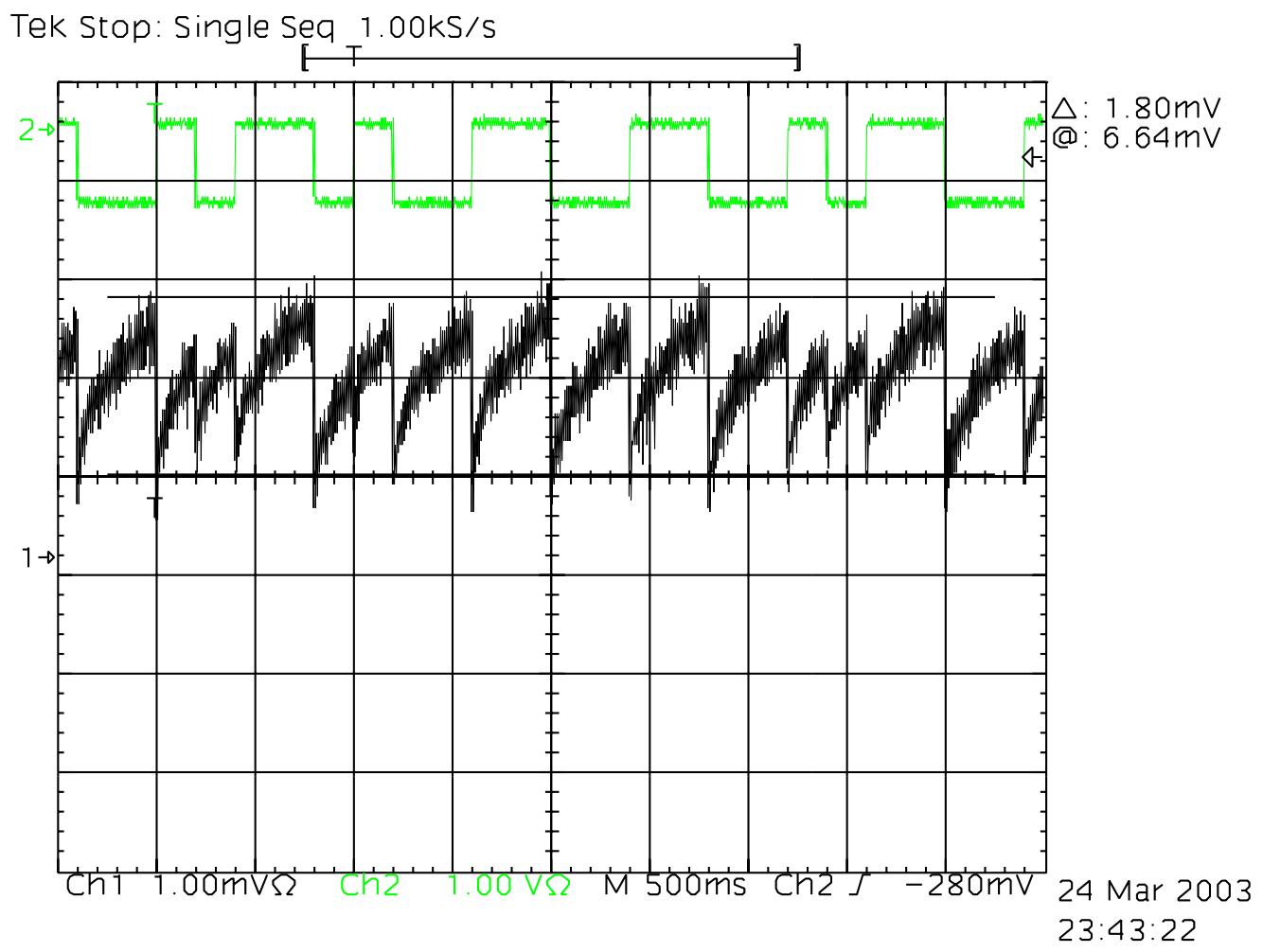

Figure 4.3. Transmission of light through crossed polarizers showing the change in PC retardation over several helicity windows (each window is $200 \mathrm{~ms}$ ). The green curve shows the helicity pattern corresponding the HV applied to the PC. The PC was being pulsed at $5 \mathrm{~Hz}$, and the horizontal scale is $500 \mathrm{~ms} /$ division.

on the PC, $R R L, L L R, L R L$, or $R L R$ with the $R R L$ and $L L R$ switches occurring with greater frequency. The outer peaks are caused by the "asymmetric" voltage change of the $R R L$ and $L L R$ pairs due to the fact that there is no voltage change before the first window of the pair.

The pair-random helicity sequence pseudorandomly chooses the helicity of the first window of every pair. This sequence produces an $A_{Q}$ multipeak structure with equally populated and broader peaks. The broadening is due to the fact that the PC history effect does not just depend on the helicity of the window previous to the pair but also on the windows previous to those, and the pair-random sequence has more of these combinations than the quad-random helicity sequence. This behavior is more explicitly shown in Figures 4.6 and 4.7 where cuts on the data are used to isolate the different peaks of the quad-random and pair-random helicity sequences, respectively. 


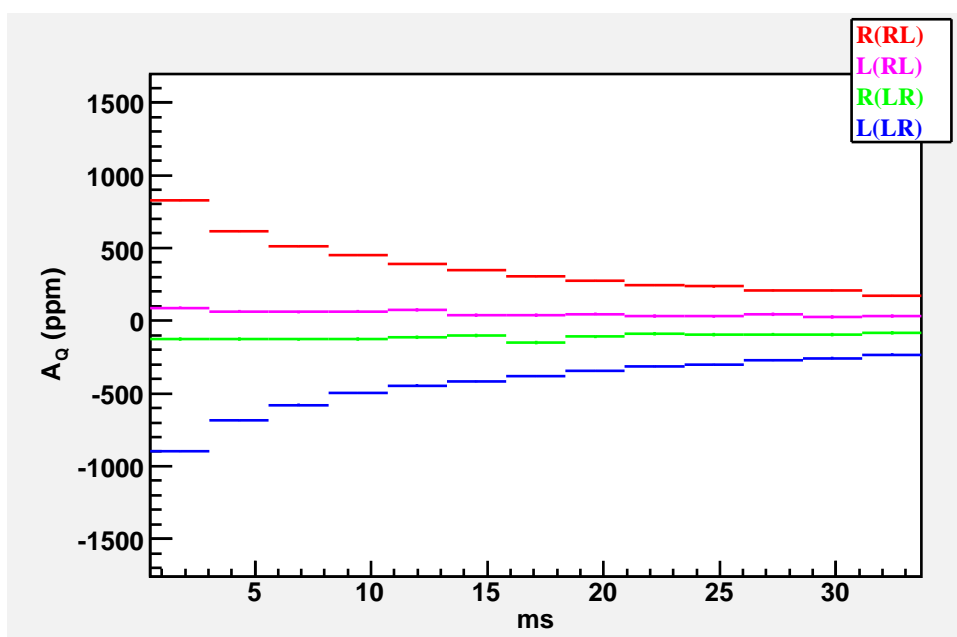

Figure 4.4. The charge asymmetry (ppm) versus time (ms) within a helicity window.

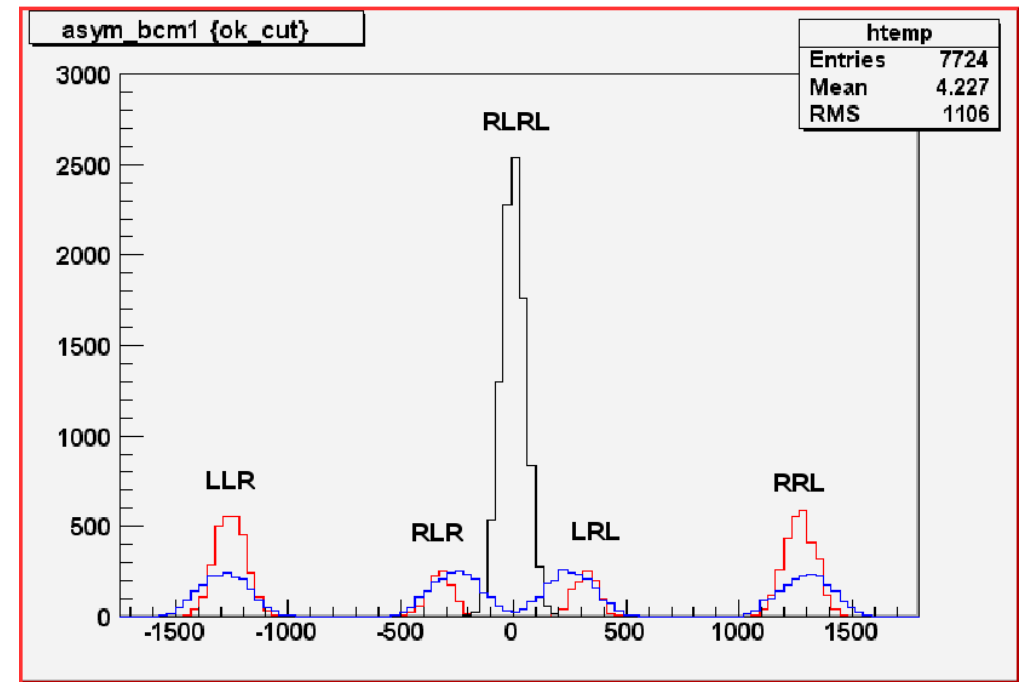

Figure 4.5. The multipeak structure of $A_{Q}$ is shown for the quad-random (red), pairrandom (blue), and pair-toggle (black) helicity patterns. The asymmetry is plotted in units of ppm.

Extensive studies were also conducted to eliminate the possibility of the HV switch or capacitance from the cables connecting the HV to the PC as a source of the history effect. We concluded that the multipeak structure was due to the intrinsic settling time of the 


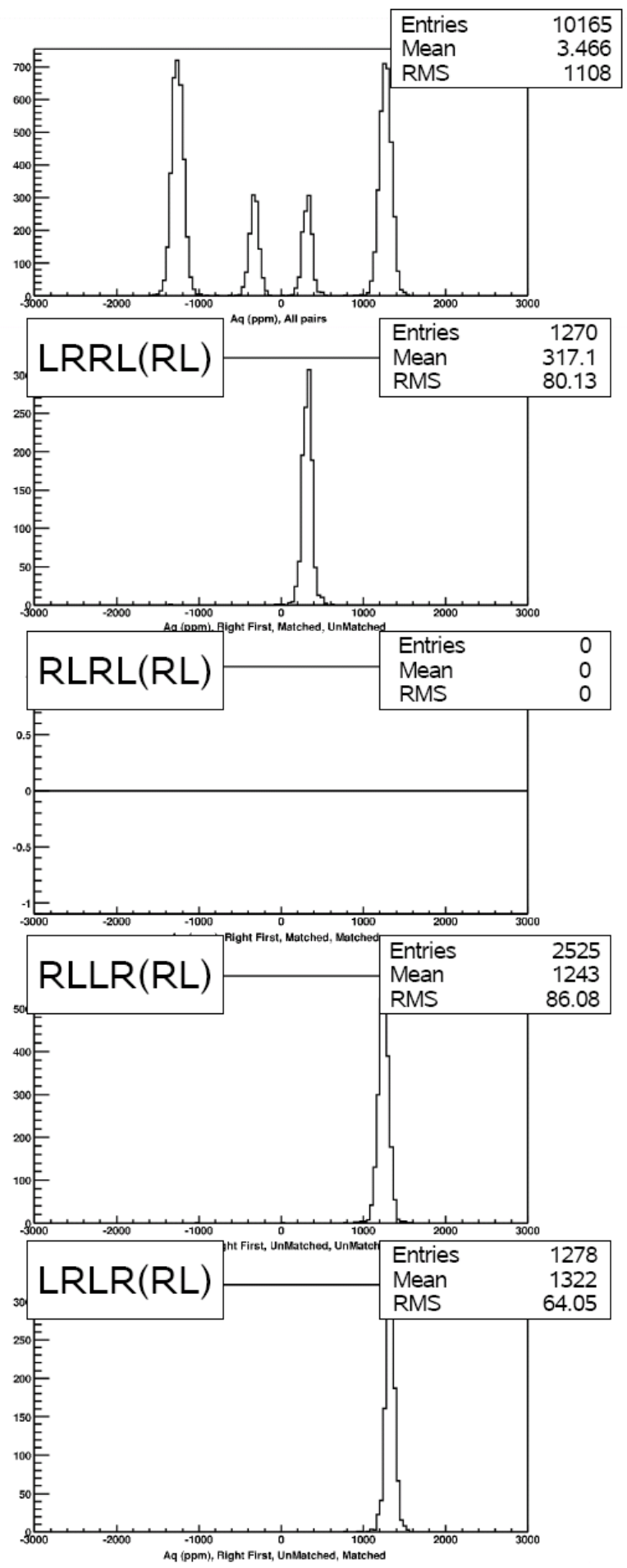

\section{Quad Random}

\section{Run 1927 \\ Matched: helicity changes before \\ first window of pair \\ UnMatched: helicity stays the same \\ for first window of pair}
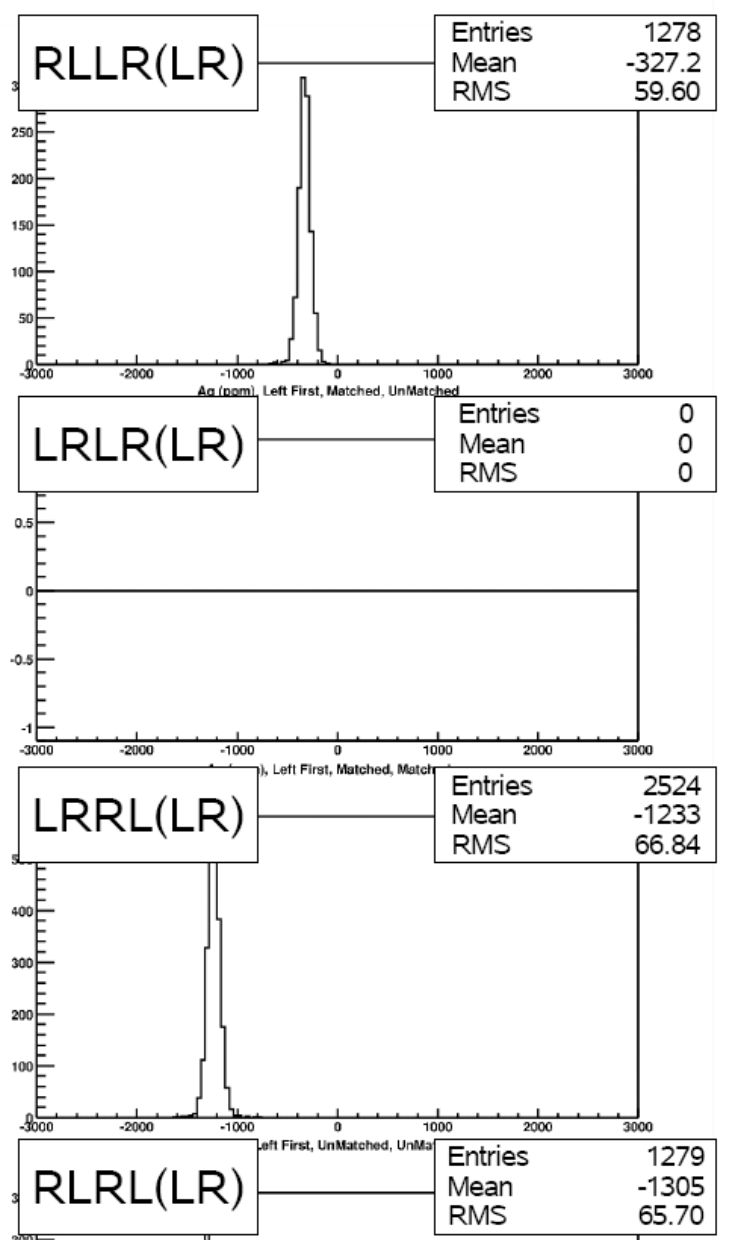

Figure 4.6. The multipeak structure of $A_{Q}$ separated into individual peaks based on the helicity of the 4 previous windows to an asymmetry pair for the quad-random helicity sequence. The $x$ axis is $A_{Q}$ in units of ppm. 


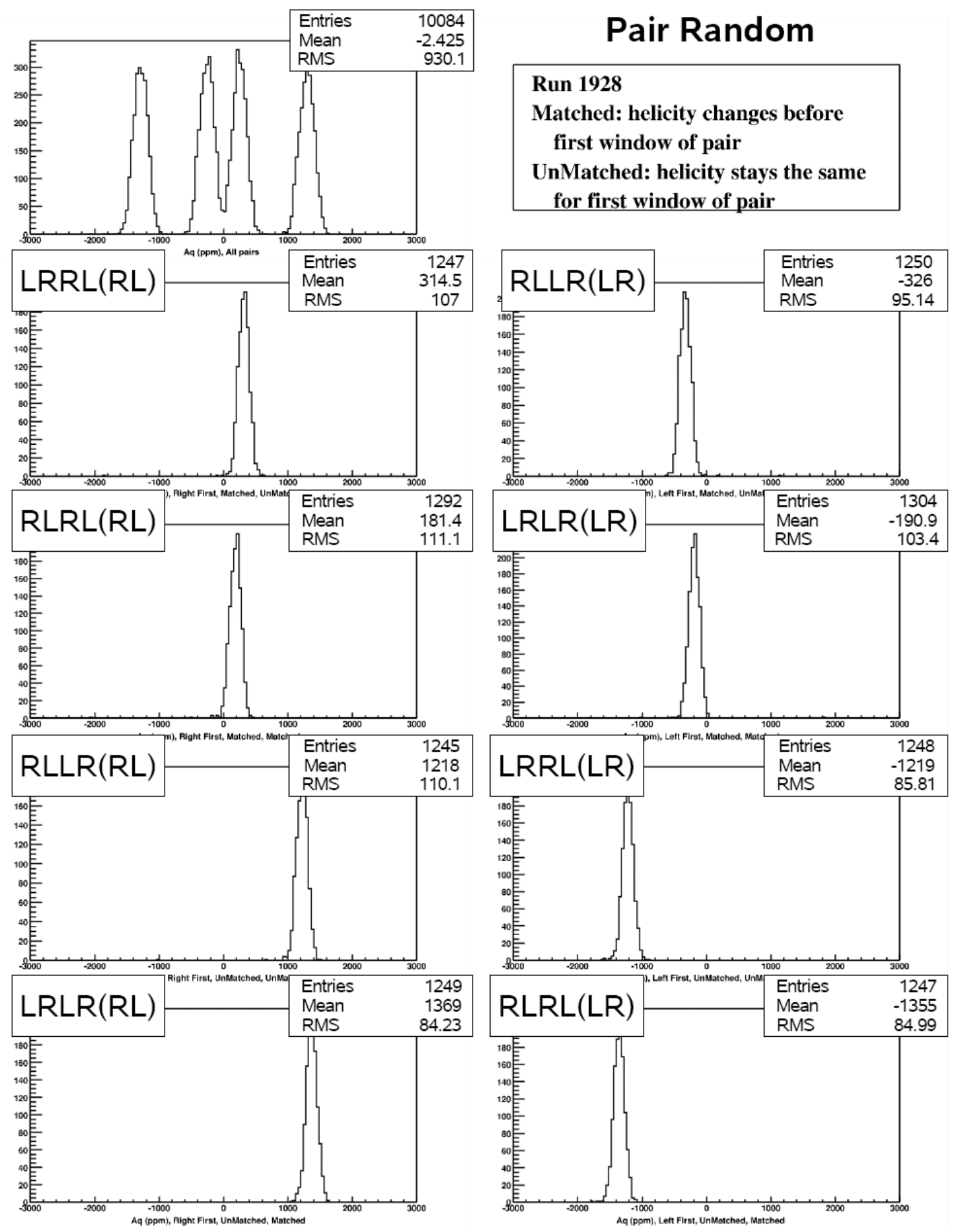

Figure 4.7. The multipeak structure of $A_{Q}$ separated into individual peaks based on the helicity of the 4 previous windows to an asymmetry pair for the pair-random helicity sequence. The $x$ axis is $A_{Q}$ in units of ppm. 


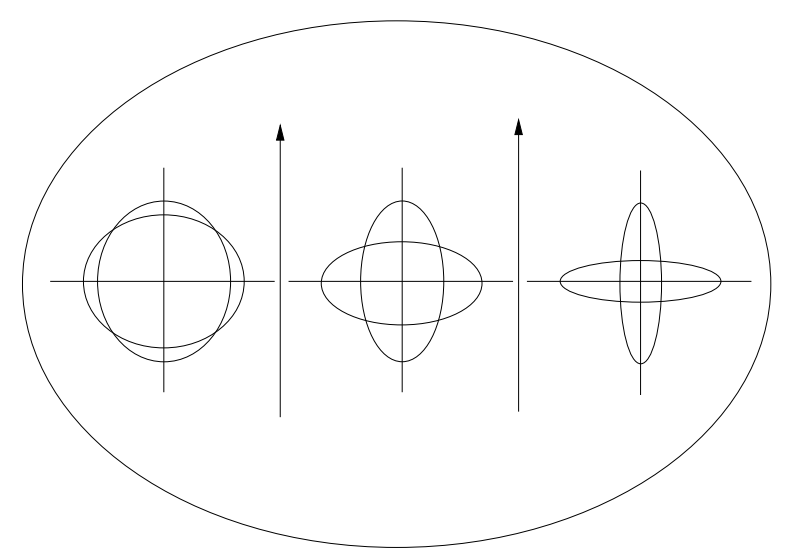

Figure 4.8. A phase gradient across the beam spot analyzed on the photocathode produces a small $A_{Q}$ on the left and large $A_{Q}$ on the right resulting in a helicity-correlated shift in the beam centroid.

Pockels cell material because the effect was evident in our standard Pockels cells as well as Pockels cells made of non-piezoelectric material.

\subsubsection{Phase Gradients}

As discussed in Section 4.1.1, a non-zero $\Delta$ phase will produce a charge asymmetry on the electron beam. Next we consider a $\Delta$ phase that varies across the laser spot which, incident on a cathode with an analyzing power, produces a charge asymmetry that varies across the electron beam spot. This effect is illustrated in Figure 4.8. If the charge asymmetry is larger on the right of the beam spot than on the left, the centroid of the beam spots for opposite helicity states will be shifted horizontally with respect to one another. In this way, the $\Delta$-phase gradient produces a helicity-correlated position difference which can be thought of as the first-moment of the charge asymmetry.

A non-zero $\Delta$ phase is caused by the residual birefringence of the PC mostly from the manufacturing of the crystal. The birefringence can be caused by stress induced from growing the crystal, cutting the crystal, and mounting the crystal in its housing. In the laser room, the charge asymmetry and position differences were measured using a biased quadrant photodiode (QPD) with each quadrant having a separate output signal. The QPD has a circular area of $\sim 0.8 \mathrm{~cm}^{2}$ which makes measurements with a large beam spot quite difficult. The QPD is mounted to a three axis translation stage so that we can align it with 


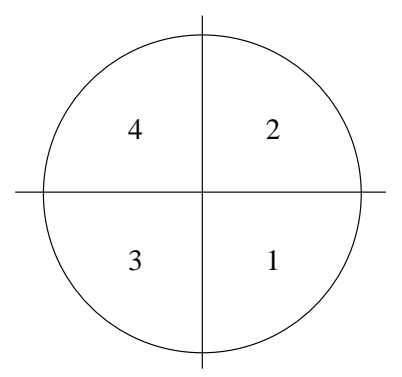

Figure 4.9. Schematic of the quadrant photodiode (QPD) showing the labels for each channel.

the beam and calibrate its position sensitivity by performing $x$ and $y$ translation scans. A schematic of the QPD is shown in Figure 4.9. The intensity is calculated as the sum of all four channels with the position calculated as follows:

$$
x=\frac{(3+4)-(1+2)}{1+2+3+4}, \quad y=\frac{(2+4)-(1+3)}{1+2+3+4} .
$$

The laser room measurements use an LP analyzer to mimic the cathode analyzing power. Because these effects are proportional to the PITA slope, the measurements in the laser room can be directly compared to the measurements we make on the electron beam. By scanning across the face of the $\mathrm{PC}$ in $x$ and $y$ directions, we can measure the $\mathrm{PC}$ phase gradients along both axes. Figure 4.10 is a plot showing the charge asymmetry and position differences for a PC $y$ translation scan. The effects plotted are produced by PC birefringence gradients measured from the Pockels cell named "Sam." These position differences are on the order of a few microns; so if we run with a PITA slope of at most $30 \mathrm{ppm} / \mathrm{V}$, we would expect position differences no worse than $500 \mathrm{~nm}$ off the cathode.

A signature of the position differences due to birefringence effects is that they flip sign with the insertion of the IHWP. This was verified in the laser room and is shown in Figure 4.11 for a PC $x$ translation scan where the IHWP "out" points (blue) are multiplied by -1 . The fact that the "in" and "out" points lie almost on top of each other after signcorrection means that the position differences are almost exclusively due to birefringence effects. 

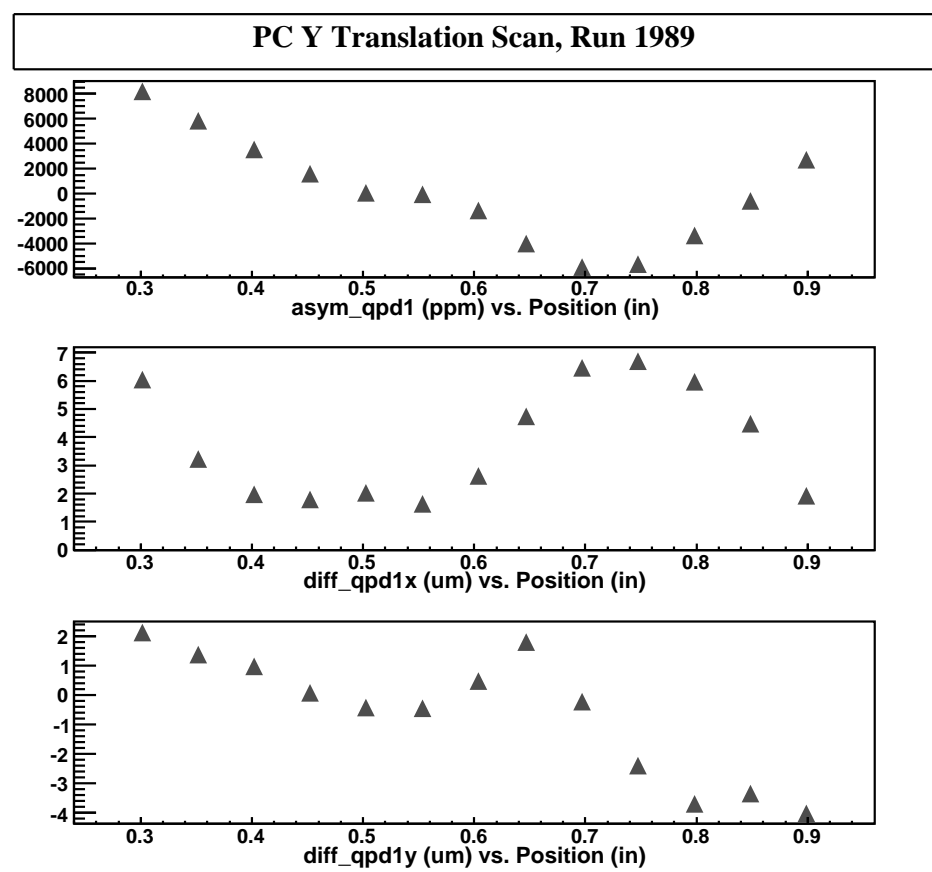

Figure 4.10. A measure of the PC birefringence gradient and associated position differences. Top: $A_{Q}(\mathrm{ppm})$, middle: $\Delta x(\mu \mathrm{m})$, bottom: $\Delta y(\mu \mathrm{m})$ versus PC $y$ position (in) of the Pockels cell "Sam."

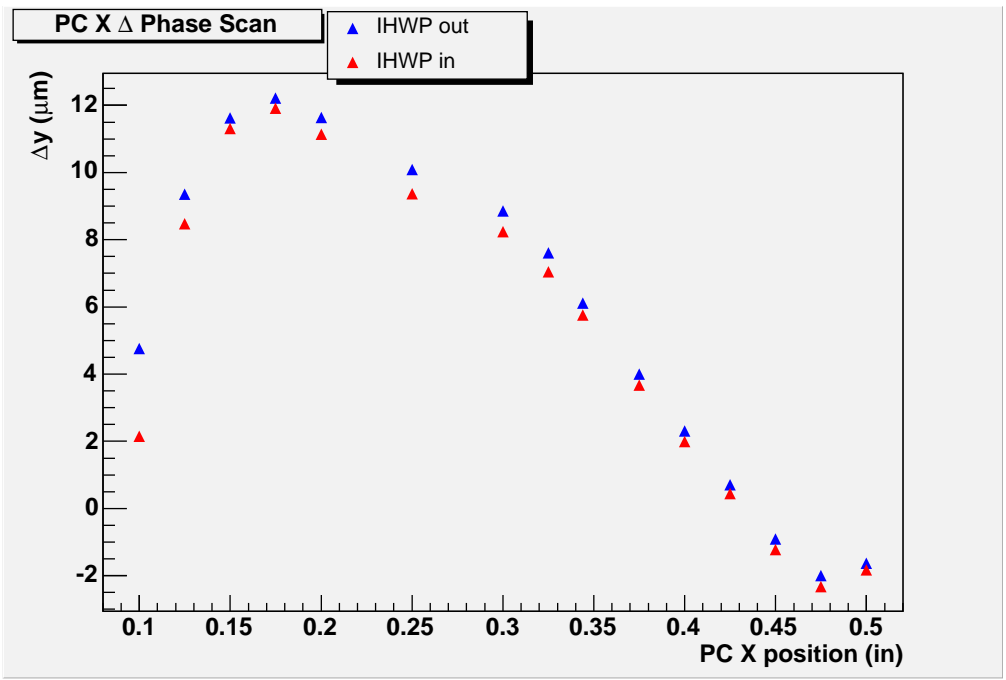

Figure 4.11. The $\Delta y(\mu \mathrm{m})$ sensitivity to birefringence gradients versus PC $x$ position (in) of the Pockels cell "Merry" for IHWP "out" (blue) and IHWP "in" (red) states. The "out" points are multiplied by -1 to show that these position differences do reverse sign with the IHWP state. 


\subsubsection{Beam Divergence}

If the Pockels cell optic axis is not well aligned with the laser beam path, the laser beam will be sensitive to the indices of refraction along all three PC axes. This results in an offset birefringence from interaction with the index of refraction of the optic axis which is not sensitive to the applied PC voltage. The birefringence offset is a function of the angle between the optic axis and the beam direction.

The divergence of the beam was identified as a source of coupling between position and the $\Delta$ phase. This is easy to understand in a ray-trace view of a diverging beam. In this picture, there is a strong correlation between the optic ray's angle and its position in the beam spot. Because the $\Delta$ phase varies with the angle of the optic ray, a correlation between the residual linear polarization and the position in the beam spot is created.

When the beam is misaligned along the optic axis, this effect creates a charge asymmetry and position differences. The sensitivity to the divergence effect was measured to be $\sim 500$ $\mathrm{nm} / \mathrm{mrad}$ by studies done in the laser room. Before special care is taken, the PC is usually aligned to the beam within a few milliradians, but this amount of misalignment produces position differences at an intolerable level for the experiment.

The pitch and yaw angles of the PC control the orientation of the PC's optic axis with respect to the beam direction; therefore, a careful scan measuring the position differences versus the pitch and yaw angles allows us to carefully align the PC optic axis to minimize the position differences due to the divergence effect. It is important to mention that a divergent beam traversing a well-aligned PC will produce a beam spot-size asymmetry while lower-order effects are minimized. This effect may be a concern for future parityviolation experiments which plan to push the precision an order of magnitude better than HAPPEX.

\subsubsection{Steering}

The Pockels cell is not only an electro-optic device, but it is also a piezoelectric device which means that its shape deforms when an electric field is applied. The PC is pulsed with either positive $\mathrm{HV}$ or negative $\mathrm{HV}$ to produce the $\pm \pi / 2$ phase shift causing the $\mathrm{PC}$ to behave as a converging or diverging lens depending on the HV applied. The lensing steers 


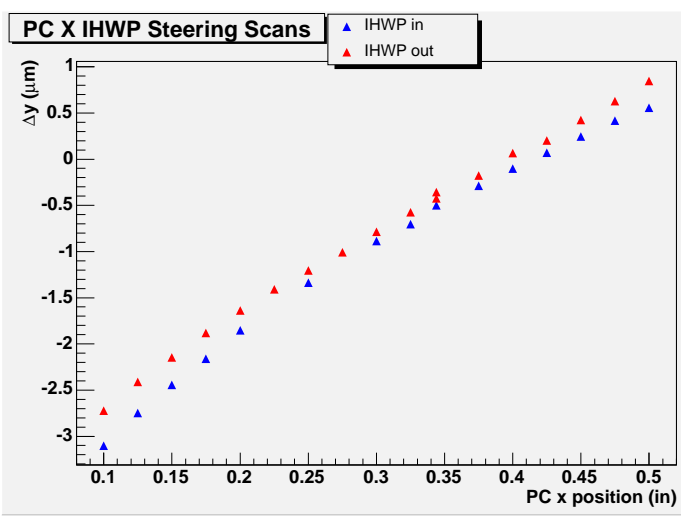

(a) IHWP steering scans

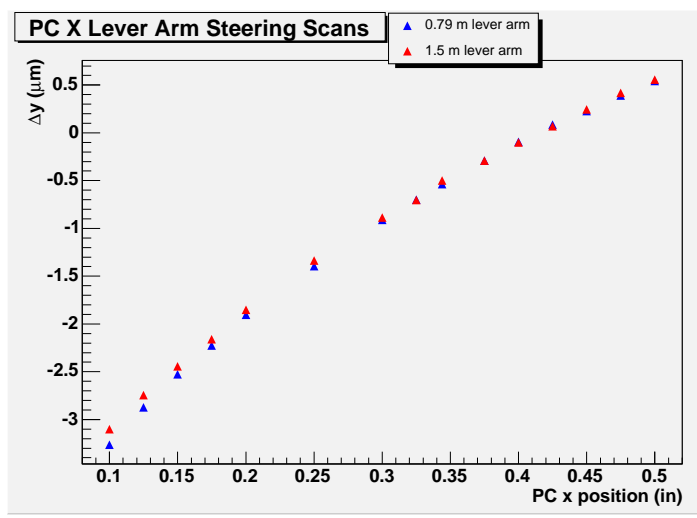

(b) Lever-arm steering scans

Figure 4.12. The position differences due to steering versus PC $x$ position for IHWP "in" (blue) and "out" (red) states are shown in (a). The position differences do not change sign with the insertion of the IHWP. The position differences plotted in (b) were measured with a lever arm of $0.79 \mathrm{~m}$ (blue) and $1.5 \mathrm{~m}$ (red) where the blue points have been scaled by the factor 1.5/0.79 to show the proper scaling for steering effects.

the beam differently for the two helicity states such that the PC behaves as a helicitycorrelated lens and produces a helicity-correlated position difference.

Steering was identified as a source of position differences using two different techniques. Without a large analyzing power in the optical setup, the position differences will be dominated by possible steering effects. Steering effects only depend on the PC HV and not the polarization state of the light; therefore, position differences due to steering will not change sign under IHWP insertion. Another signature of steering effects is that the position differences will scale with the lever arm used for the measurement. PC steering effects were verified and studied in the laser room using both of these techniques, and the results are shown in Figure 4.12.

Position differences due to steering are linearly dependent on the position the laser beam passes through the PC. If the PC is aligned well, a laser beam propagating through the center of the PC will be minimally sensitive to steering effects.

\subsubsection{Cathode Analyzing Power Gradients}

A cathode analyzing power gradient is, by definition, a QE anisotropy which changes over the surface of the cathode. Position differences arise from a non-zero $\Delta$ phase interacting 
with the analyzing power gradient. The position differences arise in much the same way as discussed in Section 4.1.2 except the interaction is between a uniform residual linear polarization on the beam interacting with a non-uniform analyzing power to create an $A_{Q}$ which varies over the beam spot resulting in a change in the beam centroid for opposite helicity states.

Although both sources of gradients produce an $A_{Q}$ that varies across the beam spot and create a helicity-correlated shift in the beam centroid, the position differences depend differently on the $\Delta$ phase. Position differences from cathode analyzing power gradients are proportional to the average $\Delta$ phase while position differences from a $\Delta$-phase gradient are independent of the average $\Delta$ phase. This difference is used as a diagnostic tool to distinguish the two effects.

\subsection{Controlling Helicity-Correlated Beam Asymmetries}

\subsubsection{Pockels Cell Characterization}

As mentioned in Section 4.1, most of the helicity-correlated beam asymmetries arise because of the Pockels cell characteristics and behavior. Our first line of defense for controlling these effects is to use a high-quality $\mathrm{PC}$ with a small residual birefringence ( $\Delta$ phase), small phase gradients, and small lensing behavior.

In the past, JLab has used PCs manufactured by Lasermetrics while SLAC used PCs manufactured by Cleveland Crystals ${ }^{1}$. For comparison, SLAC lent us one of their PCs ("Gimli"), and we proceeded to characterize all of the PCs. Gimli had a significantly smaller phase gradient $(0.34 \% / \mathrm{mm})$ than the Lasermetrics cell $(1.6 \% / \mathrm{mm})$. SLAC experiment E158 had even more stringent helicity-correlated requirements than HAPPEX such that special communication with Cleveland Crystals was used to get a good quality PC. For this reason, we contacted Cleveland Crystals to order a high quality PC for HAPPEX.

Because most PCs are used for Q-switching in lasers, parity-violation experiments are a small customer base to these manufacturers. The qualities of the PC for our needs is quite different from the Q-switches; therefore, it was important to communicate the PC qualities

\footnotetext{
${ }^{1}$ Both types of cells were $20 \mathrm{~mm}$ aperture, $\mathrm{KD}{ }^{*} \mathrm{P}$ crystals.
} 


\begin{tabular}{|l|c||c|c|c|c|c|}
\hline Type & PC Name & $A_{Q}$ grad & $\Delta x$ from grad & Steering & $A_{Q}$ Sep & $\Delta x$ sep \\
\hline \hline Lasermetrics & Merry & $1.6 \frac{\%}{\mathrm{~mm}}$ & $<30 \mu \mathrm{m}$ & $<4 \mu \mathrm{m}$ & $4 \%$ & $<0.2 \mu \mathrm{m}$ \\
& Pippin & $\mathrm{n} / \mathrm{m}$ & $\mathrm{n} / \mathrm{m}$ & $\mathrm{n} / \mathrm{m}$ & $2 \%$ & $\mathrm{n} / \mathrm{m}$ \\
\hline \hline & Gimli & $0.34 \frac{\%}{\mathrm{~mm}}$ & $\mathrm{n} / \mathrm{m}$ & $\mathrm{n} / \mathrm{m}$ & $2.5 \%$ & $\mathrm{n} / \mathrm{m}$ \\
Cleveland & Arwen & $0.25 \frac{\%}{\mathrm{~mm}}$ & $<6 \mu \mathrm{m}$ & $<2 \mu \mathrm{m}$ & $1.4 \%$ & $<0.2 \mu \mathrm{m}$ \\
Crystals & & & & & \\
& Sam & $0.21 \frac{\%}{\mathrm{~mm}}$ & $<10 \mu \mathrm{m}$ & $<3 \mu \mathrm{m}$ & $1.1 \%$ & $<0.2 \mu \mathrm{m}$ \\
& Frodo & $0.3 \frac{\%}{\mathrm{~mm}}$ & $<10 \mu \mathrm{m}$ & $<2 \mu \mathrm{m}$ & $1.7 \%$ & $<0.5 \mu \mathrm{m}$ \\
\hline
\end{tabular}

Table 4.1. A list of the Pockels cells and their properties used for characterization (where $\mathrm{n} / \mathrm{m}$ means not measured). A maximum analyzing power (PITA slope $\sim 600 \mathrm{ppm} / \mathrm{V}$ ) was used for the measurements sensitive to the PC birefringence.

we need to the manufacturer. First, in order to reduce stress induced birefringence, we ask that the PC undergoes minimal grinding and cutting and is mounted loosely in its housing. Also important is to have high extinction ratios $^{2}$ signifying small average birefringence. PCs are hygroscopic, so they are usually manufactured with windows over each end of the crystal to protect them from moisture in the air. We asked to have PCs with no windows to minimize the number of surfaces and birefringent material with which the laser beam could interact and create large and uncontrollable helicity-correlated beam asymmetries.

We named the first PC we ordered from Cleveland Crystals "Arwen," and because the PC was of such good quality, we ordered two more PCs to have for comparison and as backup PCs. A full characterization of each of the new PCs was completed and is summarized in Table 4.1.

Arwen was used for both HAPPEX 2004 and 2005 and was chosen for her small phase gradient, position differences, and $A_{Q}$ separation. It is important to choose a PC with a small phase gradient because the position differences resulting from this source do not cancel with the insertion of the IHWP. In addition, the $A_{Q}$ separation seems to be loosely

\footnotetext{
${ }^{2}$ The Pockels cell is placed between two linear polarizers, and the ratio between the maximum transmission through parallel linear polarizers and the minimum transmission through crossed polarizers defines the extinction ratio.
} 
correlated to the PC phase gradient; so choosing a PC with a small phase gradient also decreases the width of $A_{Q}$.

\subsubsection{Phase Adjustments using PITA}

As discussed in Section 4.1.1, the residual linear polarization couples to the cathode analyzing power to create a charge asymmetry (the PITA effect). An asymmetric offset voltage can be applied to the PC to change the $\Delta$ phase the PC induces on the beam. By measuring the $A_{Q}$ response to the offset voltage, we can determine the PITA slope and then set the voltage on the PC (PITA voltage) to null $A_{Q}$.

\subsubsection{The Rotatable Half-wave Plate}

The rotatable half-wave plate (RHWP) is used to control the orientation of the residual linear polarization with respect to the cathode analyzing power axis. Equation 4.2 can be extended to separately include birefringence effects from the RHWP and the vacuum window (previously these were included in the $\Delta^{0}$ term.). It is assumed that the RHWP introduces a retardation of $\pi+\gamma$ where $\gamma \ll 1$ and that the vacuum window has a birefringence $\beta \ll 1$. The resulting charge asymmetry from [44] is:

$$
A_{Q}=-\frac{\epsilon}{T}\left[\left(\Delta-\Delta^{0}\right) \cos (2 \theta-4 \psi)-\gamma \sin (2 \theta-2 \psi)-\beta \sin (2 \theta-2 \rho)\right]
$$

where $\psi$ is the orientation angle of the RHWP fast axis with respect to the horizontal and $\rho$ is the same for the vacuum window.

The first expression of Equation 4.4 is proportional to $\Delta$ and is modulated by the RHWP rotation with a $90^{\circ}$ period; therefore, the PITA slope also modulates with the same period. The second term is proportional to the RHWP birefringence and modulates with a period of $180^{\circ}$. The third term is proportional to the vacuum window birefringence. Because it is independent of $\psi$, it is responsible for a constant offset in the charge asymmetry.

For a moment, let's ignore the last two terms. If we just look at the first term, we see that we can null $A_{Q}$ very easily simply by choosing an RHWP angle that rotates the residual linear polarization produced by the $\mathrm{PC}$ to be at $45^{\circ}$ to the cathode analyzing power as illustrated in Figure 4.13. 


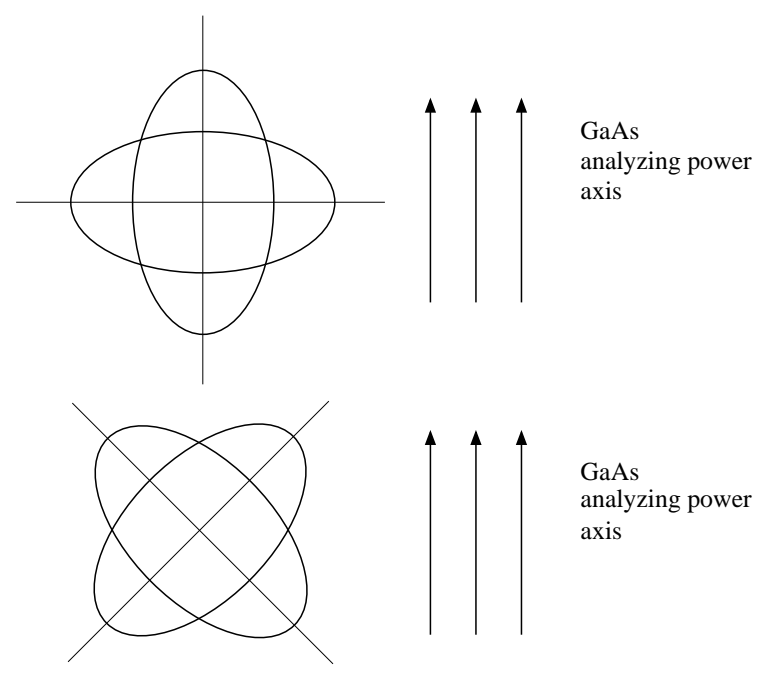

Figure 4.13. The RHWP can rotate the polarization ellipses shown in the top illustration to the orientation shown in the bottom illustration to minimize the $A_{Q}$ from the $\Delta$-phase interaction with the cathode analyzing power.

Equation 4.4 is quite convenient because it separates the contributions to $A_{Q}$ from each of the optical elements. This feature becomes very useful for using the RHWP as a diagnostic tool to understand the sources of $A_{Q}$ while also giving us the ability to control our sensitivity to them.

The RHWP can be rotated so that we eliminate the sensitivity of the PC birefringence to the cathode analyzing power. In practice, we choose the RHWP angle to provide a small, but non-zero PITA slope. Having some sensitivity to analyzing power allows us to use PITA voltage to cancel the effects of residual linear polarization produced by the vacuum window or the RHWP itself while also reducing the position differences from the PC $\Delta$-phase gradients.

The choice of an RHWP angle where the PITA slope is small additionally reduces the $A_{Q}$ separation and position differences due to birefringence gradients. For Arwen, the $A_{Q}$ separation was 14000 ppm for a PITA slope of 600 ppm/V. During HAPPEX, we ran with a PITA slope $\leq 8 \mathrm{ppm} / \mathrm{V}$ which makes the separation negligible $(<200 \mathrm{ppm})$ compared to the natural laser jitter. The resulting $A_{Q}$ distribution is Gaussian with no obvious distortion from the separation. With this PITA slope, the position differences due to phase gradients were expected to be no greater than $300 \mathrm{~nm}$ in the injector. 


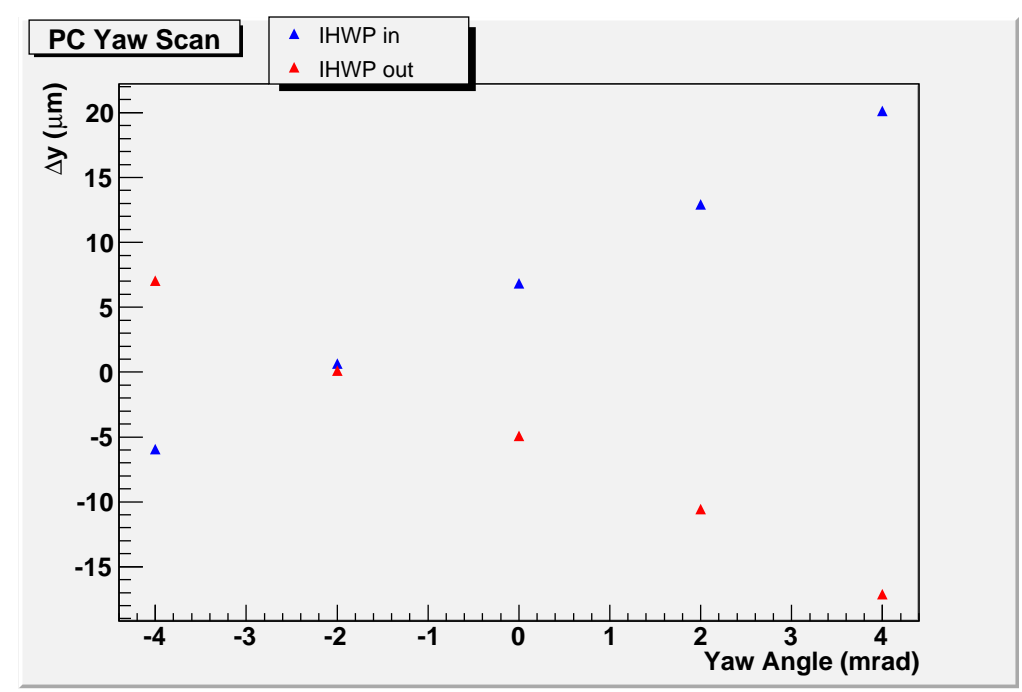

Figure 4.14. Position difference response to PC yaw angle for both IHWP states (red: IHWP in, blue: IHWP in).

\subsubsection{Minimizing Divergence Effects}

In order to minimize position differences that arise from the beam divergence coupling to the $\mathrm{PC} \Delta$ phase, we measure the sensitivity of the position differences to the PC pitch and yaw angles for each IHWP state. We enhance the size of these position differences for measurement by using an LP analyzer. It is possible to find the pitch and yaw angles to give the best average alignment of the PC optic axis with the laser beam direction. Scans from the 2005 source setup are shown in Figure 4.14 where a change of only 2 mrad in yaw was necessary to minimize these position differences.

\subsubsection{Minimizing Steering}

In describing the $\mathrm{PC}$ as a helicity-correlated lens, it is obvious that centering the beam through the "lens" will minimize the position differences from steering. The centering is done by measuring the sensitivity of the position differences to the PC translation. The results from Arwen are shown in Figure 4.15. A sign that the position differences are due to steering is that they are linearly dependent on the PC translation such that a linear fit to the data reliably predicts the $\mathrm{PC}$ position for minimizing steering. One observation which 


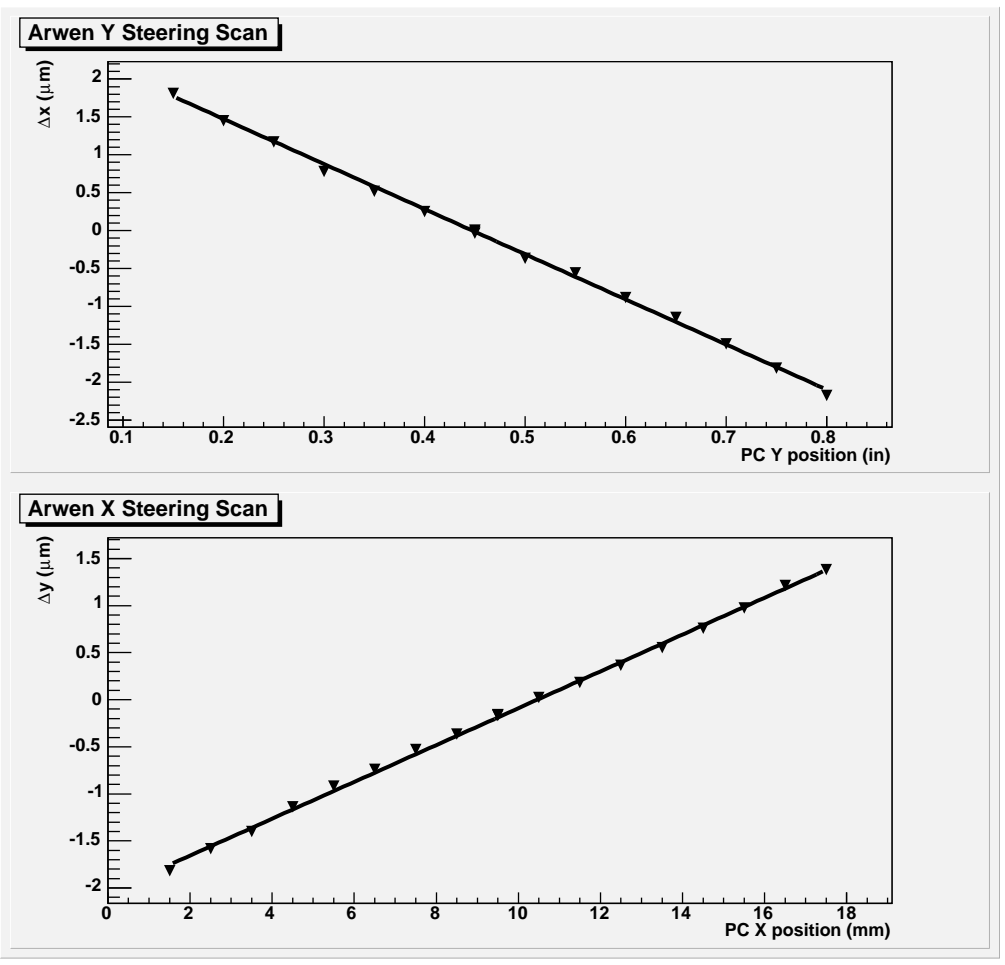

Figure 4.15. Position differences versus PC translation for Arwen.

is not understood is that the translation of the $\mathrm{PC}$ in the $x$ and $y$ directions affects the $\Delta y$ and $\Delta x$ position differences respectively.

Another method used by SLAC E158 for minimizing steering is imaging the beam at the exit of the PC onto the cathode so that all angle information is removed from the beam [44]. This technique was not used at JLab because no suitable solution could be found to satisfy all the existing constraints of the source.

\subsubsection{Insertable Half-wave Plate}

The IHWP is located upstream of the PC and is used to rotate the orientation of the polarization incident on the $\mathrm{PC}$ by $90^{\circ}$. This rotation reverses the sign of the beam helicity relative to the electronic helicity signals. This reversal cancels any asymmetries created by electronic pickup of the helicity signal.

Any helicity-correlated beam asymmetry produced by a polarization effect will flip sign with the IHWP insertion and will not cancel. Position differences due to steering do not 
change sign because they are only dependent on the HV applied to the PC and not the helicity and so will cancel with IHWP insertion.

\subsubsection{Feedback}

An active feedback on charge is used only after all of the above techniques have been exhausted. In particular, an intensity attenuator (IA) feedback system consisting of a PC and LP acts as an electro-optic shutter, to make small corrections to $A_{Q}$ on a short timescale. Feedback does not do anything to solve the source of the problem; therefore it should not be used to make large corrections which could cause higher-order effects.

PITA feedback is used to avoid having the IA make large corrections because it does address the source of the problem by adjusting the residual linear polarization on the beam.

\subsubsection{Other Methods for Control}

Another method to reduce position differences due to phase gradients or cathode analyzing power gradients is to reduce the beam spot size at the PC. This can be accomplished by placing a beam waist at the PC by using a telescope in the beamline upstream of the PC.

A reduction in helicity-correlated position differences can also be obtained from the adiabatic damping of phase space as the beam is accelerated. Theoretically, this factor can be as large as $\sqrt{p_{0} / p}$ which is $\sim 95$ for HAPPEX. Without special care, this benefit is lost due to excessive beam correlation to poorly configured optics. At JLab, factors of less than 10 are typical; however, a dedicated effort by accelerator physicists resulted in a stable, well-matched optical tune which provided factors $>25$ for the entire 2005 run of HAPPEX-H.

\subsection{Source Setup}

A detailed description of the source setup procedure, with special attention paid to the Pockels cell alignment procedure, is included in the appendix of this thesis. During the 2004 source setup, we spent between 12 and 16 hours setting up the polarized source optics.

The setup took slightly longer than we wanted in order to get satisfactory results because 
we observed effects on the laser beam that were not present while performing our studies in the laser room. In this section, I will discuss the unexpected issues that we faced during the setup period.

\subsubsection{Beam Waist}

The first issue we faced was in properly placing the beam waist at the PC while maintaining the beam spot size at the cathode. We had practiced this procedure in the laser room without issue using the same beam telescope as we were using in the source; but we could not replicate these results. This is probably due to the fact that the laser room does not have a full mock-up of the source. We had to choose between having a waist at the PC and a large beam spot at the cathode or a larger beam spot at the PC with a small beam spot at the cathode. We decided that we would run with the small (500 $\mu \mathrm{m})$ spot on the cathode because although this increases our sensitivity to phase gradients in the PC, it should minimize the beam spot through the vacuum window for which we have no control.

In 2005 , we actually made the opposite decision and ran with a waist at the $\mathrm{PC}(\sim 700$ $\mu \mathrm{m})$ and a $900 \mu \mathrm{m}$ spot on the cathode. The reason for this decision was two-fold. For one, this setup would minimize our sensitivity to PC sources of position differences and keep us from digging a deep QE hole in the superlattice cathode (as discussed in Section 4.5) and perhaps increase the lifetime of the cathode.

\subsubsection{Steering}

The next big issue we faced in the tunnel was trying to sort out the sources of position differences. When we centered the PC to null position differences from steering, we measured a very small PITA slope, but non-zero. We centered the PC for the IHWP "out" state and achieved position differences $<200 \mathrm{~nm}$. We were shocked when we put the IHWP in and saw position differences of $1.4 \mu \mathrm{m}$. After some time, we concluded that this had to be due to a polarization effect even though we did not know the source of it. In order to balance steering and non-steering effects with no analyzer in the beamline, we found the location

through the PC which made the position differences equal and opposite in sign for the two IHWP states such that steering effects would cancel under IHWP insertion. I should note 
that this effect was seen during the source setup for Gun 3 (superlattice) and for the Gun 2 (strained-layer) setup, but it was not understood during the Gun 3 setup; and therefore was not minimized appropriately which enhanced the position differences measured during the beginning of the experiment.

Leading up to HAPPEX 2005, we made an effort to "find" and study this effect in the laser room. In the end, that was not difficult and this effect was understood as the divergence effect discussed in Section 4.1.3. As a result, our PC alignment procedure was changed to minimize this effect.

\subsubsection{Injector Source Setup}

The injector setup involves performing four RHWP scans (described in Section A.2.2 to obtain the electron beam data necessary for selecting the optimal RHWP angle for minimizing $A_{Q}$ and position differences simultaneously. This procedure takes approximately two hours with stable beam delivery. In this section I summarize the results of the Gun 2 setup with the strained-layer cathode because we were able to perform a full injector source setup during this installation period. The charge asymmetry measured as a function of the RHWP angle for both IHWP states is shown in Figures 4.16 and 4.17 for no PITA voltage offset and a PITA offset of $-200 \mathrm{~V}$, respectively, applied to the PC.

There are several features to note from the plots in these figures. Firstly, there is a large constant offset term in the $A_{Q}$ functional fit which changes sign perfectly with IHWP. This strongly suggests a significant vacuum window birefringence which required running with a significant PITA slope to zero the offset. In Figure 4.16, the fact that the $4 \theta$ amplitude is small ( 700 ppm) and flips sign with the IHWP suggests that the PC voltages were tuned quite well. In contrast, when a PITA voltage of -200 V was applied to the PC (Figure 4.17), the amplitude of the $4 \theta$ term was $\sim 12000 \mathrm{ppm}$.

The position differences versus RHWP angle are shown in Figure 4.18 for both IHWP states and no PITA voltage offset ( $\Delta x$ is shown in the top of the figure and $\Delta y$ in the bottom). The $4 \theta$ amplitude is small and flips sign for both sets of position differences which is indicative of PC phase gradients, polarization gradients upstream of the PC, and analyzing power gradients. The position differences versus RHWP angle for a PITA voltage 


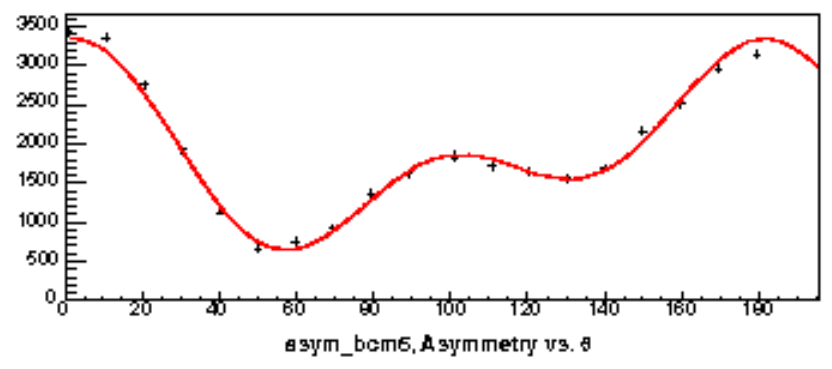

$\mathrm{Aq}=188320+897.33 \mathrm{zln}(2 \mathrm{x}+110.60)+67540 \mathrm{zln}(4 \mathrm{x}+66.97)$

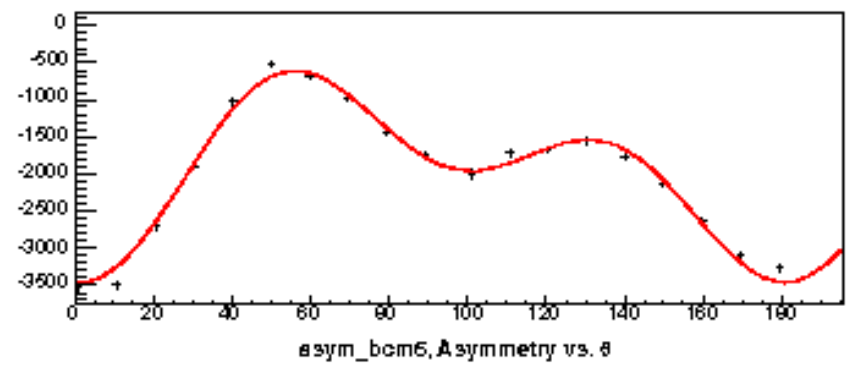

$A q=-1923.12+-905.36 z \ln (2 x+112.80)+-743.70 z \operatorname{lo}(4 x+7128)$

Figure 4.16. $A_{Q}$ (ppm) versus RHWP angle (deg.) measured in the $5 \mathrm{MeV}$ injector region for both IHWP states (top: IHWP out, bottom: IHWP in) and PITA voltage $=0 \mathrm{~V}$.

offset of $-200 \mathrm{~V}$ are shown in Figure 4.19. The $4 \theta$ amplitude is large for both $\Delta x$ and $\Delta y$ which suggests a large analyzing power gradient, most likely on the cathode.

Noticeably though, $\Delta x$ is offset from 0 and flips sign with IHWP which is due to the vacuum window birefringence interaction with the cathode analyzing power gradient or vacuum window birefringence gradients. The offsets for $\Delta y$ position differences are not so easily explained. It is possible that the $\Delta y$ offsets are a combination of vacuum window and steering effects.

In the end, it was possible to find an RHWP angle that satisfied our constraints. In particular, we chose to run with an RHWP angle of $73^{\circ}$ which zeros $\Delta x$ and reduces $\Delta y$ while providing a PITA slope large enough $(\sim 7.5 \mathrm{ppm} / \mathrm{V})$ to zero the $A_{Q}$ offset for both IHWP states. Because we had to run with a large PITA slope to correct for the vacuum window, we were less able to tune the RHWP to eliminate the PC birefringence gradient 

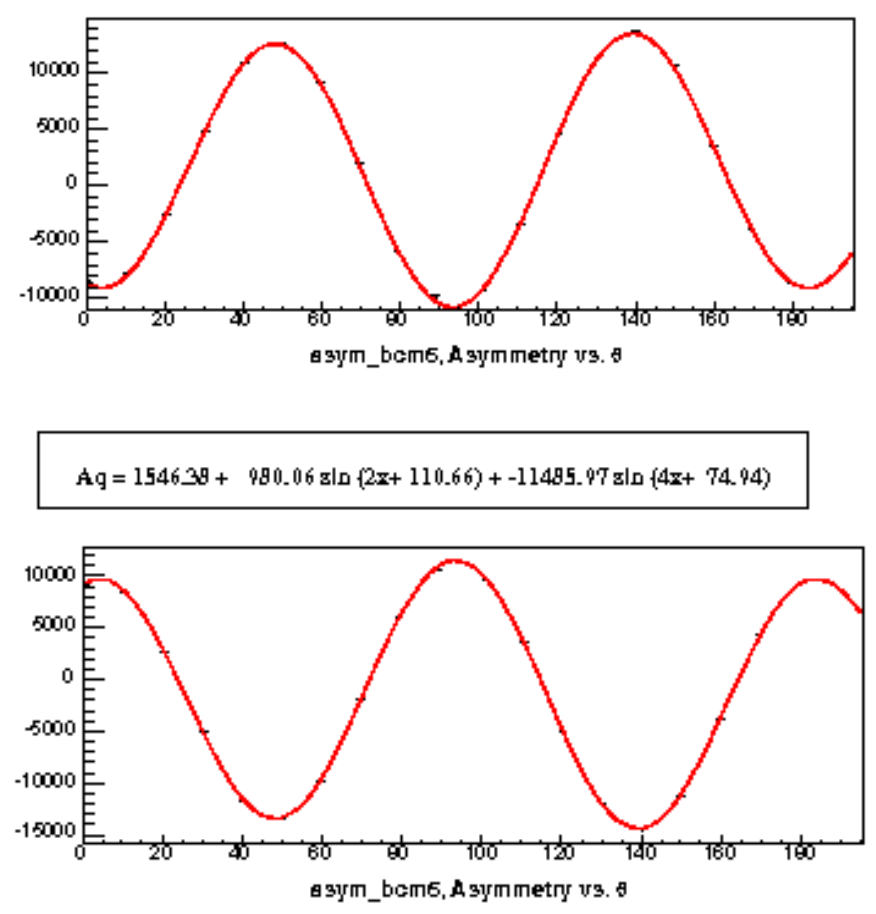

$A q=-1639.70+-1009.57 \mathrm{gln}(2 \mathrm{x}+112.03)+122.58 .35 \mathrm{gln}(4 \mathrm{z}+74.82)$

Figure 4.17. $A_{Q}$ (ppm) versus RHWP angle (deg.) measured in the $5 \mathrm{MeV}$ injector region for both IHWP states (top: IHWP out, bottom: IHWP in) and PITA voltage $=-200 \mathrm{~V}$.

effects, so it was vital that we used the PC Arwen which has a very small birefringence gradient.

\subsection{Helicity-Correlated Feedback}

Two types of active feedback were used during the 2004 HAPPEX run in order to control the charge asymmetry on target. IA feedback was used on a two-minute timescale while PITA feedback was used on an "as needed" basis. Because of crosstalk and bleedthrough from the Hall $\mathrm{C}$ beam on the Hall A beam, it was also necessary to perform IA feedback on the Hall $\mathrm{C}$ beam.

The PITA slope (for both IHWP states) and IA slopes were measured during the setup of the source and occasionally during the experiment to maintain the quality of feedback. 

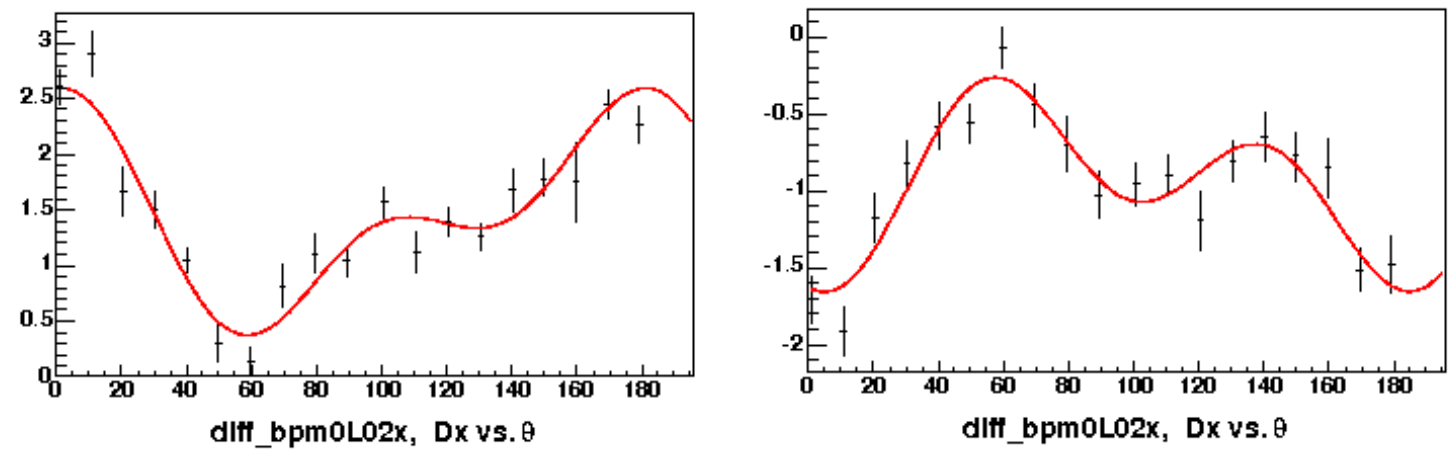

$D x=1.45+0.79 \operatorname{stn}(2 x+116.56)+0.49 \operatorname{stn}(4 x+62.33)$

$\mathrm{Dx}=-0.93+-0.37 \sin (2 x+110.58)+-0.42 \sin (4 x+57.67)$
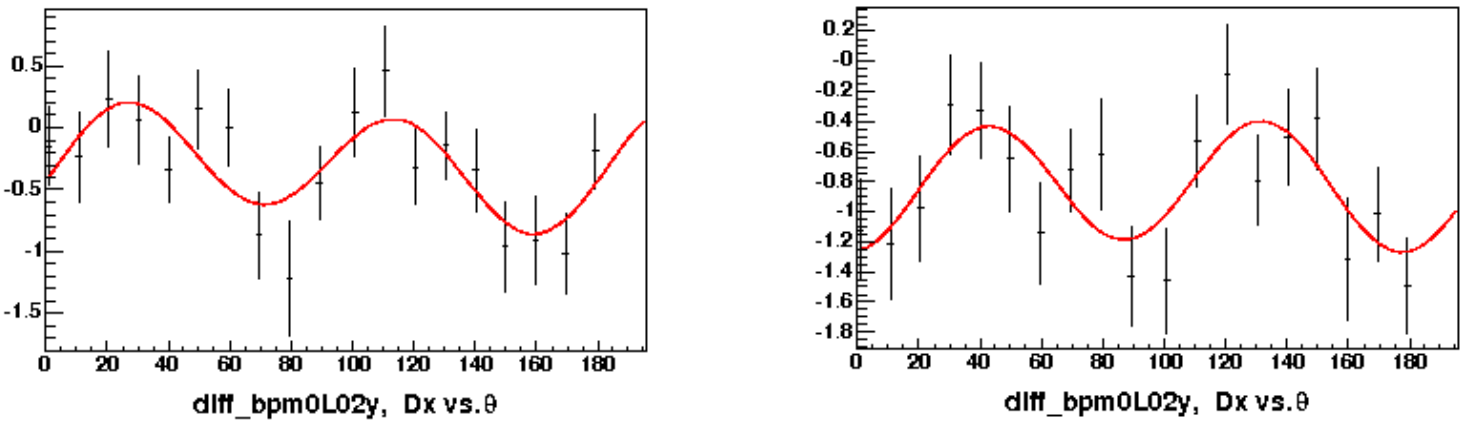

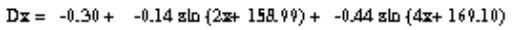

$D x=-0.22+-0.05 \operatorname{stn}(2 x+7284)+-0.41 \operatorname{stn}(4 x+100.85)$

Figure 4.18. The top plots are $\Delta x(\mu \mathrm{m})$ versus RHWP angle (deg.) measured in the 5 $\mathrm{MeV}$ injector region for both IHWP states (left: IHWP out, right: IHWP in) and PITA voltage $=0 \mathrm{~V}$. The bottom plots are the same as the top but for $\Delta y(\mu \mathrm{m})$.

In addition, if the Hall $\mathrm{C}$ IA feedback was unable to zero their $A_{Q}$, it was necessary to adjust the IA waveplate angle such that the IA system had a greater dynamic range.

\subsubsection{Hall A IA Feedback}

During 2004, IA feedback was used during production running to control $A_{Q}$. A feedback script interfaces the DAQ and EPICS to monitor $A_{Q}$, readback the IA parameters and set new values for the IA parameters. IA feedback works by operating the IA cell at ground for one helicity state and at some small voltage in the other helicity state. 

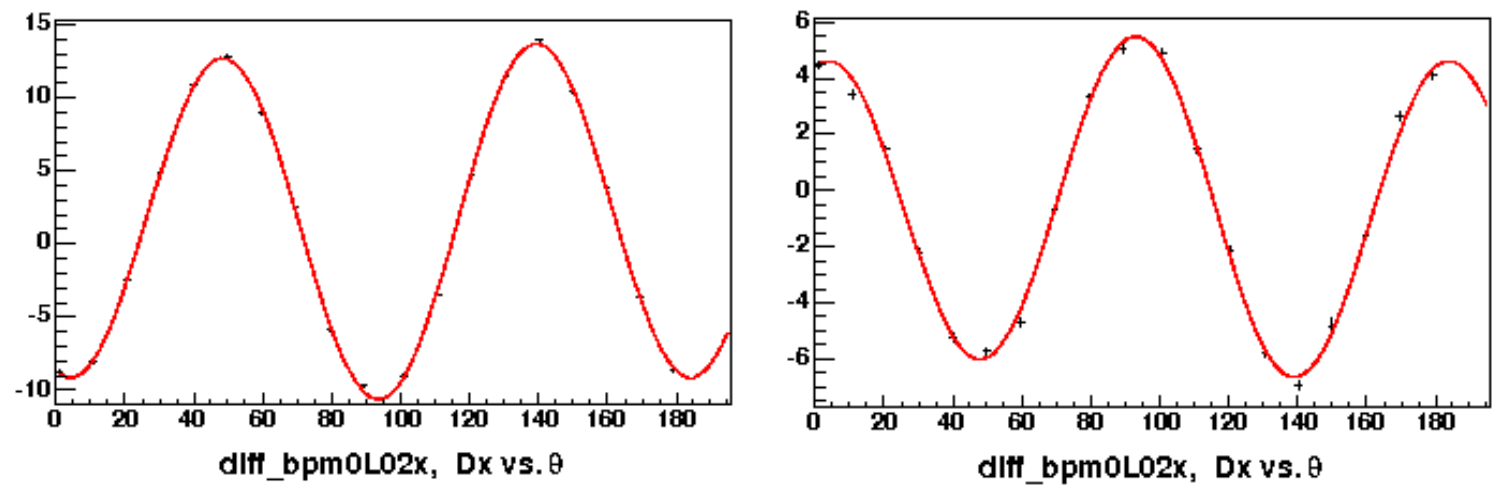

$D x=1.66+0.83 \operatorname{stn}(2 x+115.60)+-11.52 \operatorname{stn}(4 x+74.29)$

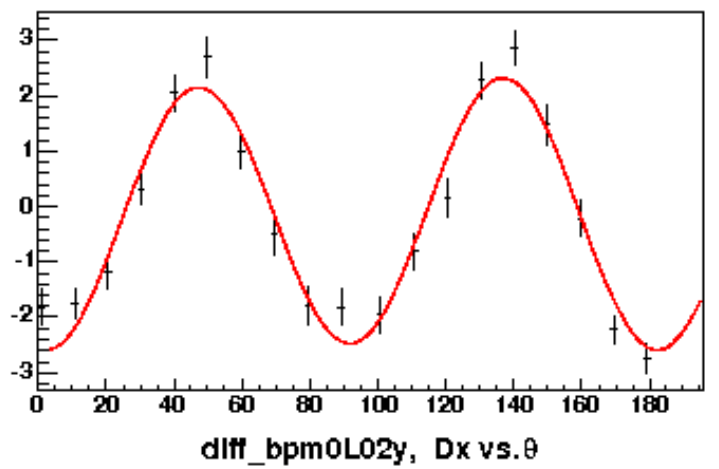

$D x=-0.64+-0.54 \sin (2 x+118.95)+5.70 \sin (4 x+75.90)$

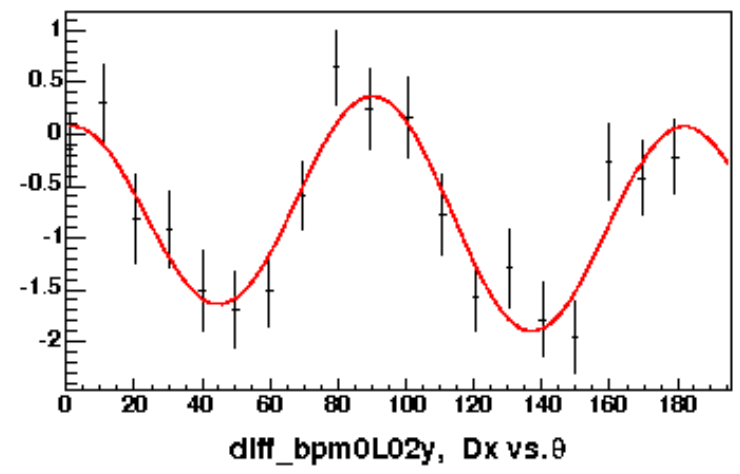

$\mathrm{Dx}=-0.15+-0.10$ stn $(2 \mathrm{x}+31.44)+-2.39$ stn $(4 \mathrm{x}+81.53)$

$\mathrm{Dx}=-0.77+-0.20 \mathrm{~s} \ln (2 \mathrm{x}+128.80)+0.995 \ln (4 \mathrm{x}+85.50)$

Figure 4.19. The top plots are $\Delta x(\mu \mathrm{m})$ versus RHWP angle (deg.) measured in the 5 $\mathrm{MeV}$ injector region for both IHWP states (left: IHWP out, right: IHWP in) and PITA voltage $=-200 \mathrm{~V}$. The bottom plots are the same as the top but for $\Delta y(\mu \mathrm{m})$.

The voltage of the IA cell operates over a range of $\pm 240 \mathrm{~V}$ for a DAC V setting between 1 and 9 where 5 corresponds to $0 \mathrm{~V}$. The IA cell then acts as a variable wave retarder for one helicity state and the light is analyzed by an LP downstream of the IA cell transmitting more or less light for that state in order to equalize the light between the two states of a pair. In practice we operate the IA cell between 4 and $6 \mathrm{DAC} \mathrm{V}$ because we want to minimize the position differences that can be induced by operating the $\mathrm{PC}$ at higher voltages. Also, 
the necessity for the IA to operate at larger voltages means the helicity PC voltages are no longer optimized for minimizing $A_{Q}$.

\subsubsection{PITA Feedback}

IA feedback is the brute force method for minimizing the charge asymmetry. PITA feedback is used to adjust the amount of residual linear polarization on the beam in order to minimize $A_{Q}$. By using PITA feedback, we address the source of the problem creating $A_{Q}$ and minimize the use of IA feedback. PITA feedback uses the PITA slope to make adjustments to the helicity $\mathrm{PC}$ voltage to zero $A_{Q}$. Because PITA feedback adjusts the beam polarization to minimize $A_{Q}$, it is important to verify that the $A_{Q}$ for which the IA is correcting is real and not a consequence of clipping the beam on injector apertures; therefore, PITA feedback should only be used when transmission of the beam through the accelerator is high. During the 2005 HAPPEX run on hydrogen, PITA feedback was the only active charge feedback used, and it operated on a two-minute timescale.

\subsubsection{Hall C IA Feedback}

The Hall C IA feedback worked similarly to the Hall A feedback described in Section 4.4.1 with a few differences. The Hall $\mathrm{C}$ feedback operated on a five-minute rather than a two-minute timescale and operated over a much larger dynamic range. The helicity PC is optimized for the Hall A beam and because the Hall C laser wavelength and polarization orientation incident on the $\mathrm{PC}$ may be different from Hall $\mathrm{A}$, the Hall $\mathrm{C} A_{Q}$ can be considerable. For this reason, we run the Hall C IA system with as large a dynamic range as necessary and let the IA voltage operate over the full range of 1-9 DAC V.

For HAPPEX, the goal was to keep the Hall C $A_{Q}$ less than $100 \mathrm{ppm}$ at any given time. For two reasons, the Hall $\mathrm{C}$ feedback was not as reliable as we would have liked. One reason was that the Hall $\mathrm{C} \mathrm{BCM}$ has very low resolution which made it difficult to reliably decipher when beam was on or off in the hall. The second reason was that it required careful attention by shift workers and experts to recognize when $A_{Q}$ was drifting away from the desired value and when more dynamic range was needed from the IA system to null $A_{Q}$. 


\begin{tabular}{|c|r|r|}
\hline & \multicolumn{1}{|c|}{2004} & \multicolumn{1}{|c|}{2005} \\
\hline \hline$\left\langle A_{Q}\right\rangle$ & $2.6 \mathrm{ppm}$ & $0.406 \mathrm{ppm}$ \\
$\left\langle A_{E}\right\rangle$ & $21.6 \mathrm{ppb}$ & $0.2 \mathrm{ppb}$ \\
$\langle\Delta x\rangle$ & $-7.9 \mathrm{~nm}$ & $0.5 \mathrm{~nm}$ \\
$\langle\Delta y\rangle$ & $1.1 \mathrm{~nm}$ & $1.7 \mathrm{~nm}$ \\
$\left\langle\Delta x^{\prime}\right\rangle$ & $4.2 \mathrm{nrad}$ & $-0.2 \mathrm{nrad}$ \\
$\left\langle\Delta y^{\prime}\right\rangle$ & $1.2 \mathrm{nrad}$ & $0.2 \mathrm{nrad}$ \\
\hline
\end{tabular}

Table 4.2. Average helicity-correlated beam asymmetries for HAPPEX-H.

\subsection{Helicity-Correlated Measurements during HAPPEX}

The average helicity-correlated beam asymmetries measured during HAPPEX 2004 and 2005 are shown in Table 4.2. Although the 2004 values are larger than our goal for the full statistics of the experiment, they were adequate for the data set because we could tolerate a larger systematic error with a larger statistical error. Because of a careful setup of the polarized source, use of position feedback was not needed despite the instabilities in position differences caused by issues with the superlattice cathode and beam crosstalk. The 2005 helicity-correlated beam asymmetries surpassed our expectations due to a well-aligned source and good transport of the beam through the accelerator. For the 2005 hydrogen run, the control of the helicity-correlated beam asymmetries was also improved because Hall $\mathrm{C}$ was not running; therefore, we did not have to worry about beam crosstalk off of the cathode

In 2004, The superlattice cathode was used during the HAPPEX- ${ }^{4}$ He run and for the first 24 of 57 slugs of HAPPEX-H. We switched to Gun 2 with the strained-layer cathode during the July 4 accelerator down period.

The superlattice cathode lifetime was quite poor which was not a problem during HAPPEX- $-{ }^{4} \mathrm{He}$ because Hall A was the only high-current hall running, but it became a serious problem during HAPPEX-H because both Halls A and C needed high current. Figure 4.20 (top) shows the QE over the area of the superlattice cathode at the end of June 2004. The beam location on the cathode had to be changed frequently (once to twice a day) during HAPPEX-H (summarized in Table 4.3) to maintain production current needed for Halls A and C. Because the superlattice cathode was not anodized, it had a larger active 


\begin{tabular}{|c|c|c|c|c|}
\hline Experiment & Date & Time & $x$ & $y$ \\
\hline \hline \multirow{3}{*}{${ }^{4} \mathrm{He}$} & June 1 & & 1000 & 1170 \\
& June 18 & $09: 49$ & 950 & 1300 \\
& June 22 & $09: 55$ & 1050 & 1300 \\
\hline \hline \multirow{3}{*}{$\mathrm{H}$} & June 26 & $08: 30$ & 1100 & 1200 \\
& June 27 & $10: 50$ & 1100 & 1100 \\
& June 28 & $09: 00$ & 1150 & 1000 \\
& June 28 & $16: 00$ & 1100 & 1150 \\
& June 29 & $08: 25$ & 1125 & 1175 \\
& June 29 & $17: 50$ & 1100 & 1250 \\
\hline
\end{tabular}

Table 4.3. A list of the beam spot locations on the superlattice cathode during HAPPEX 2004 production running. The cathode lifetime decreased significantly during the HAPPEXH run.

area $\left(\sim 123 \mathrm{~mm}^{2}\right)$ than the strained-layer cathode $\left(\sim 20 \mathrm{~mm}^{2}\right)$ and probably contributed to the poor cathode lifetime. The QE of the strained-layer cathode is shown in the bottom of Figure 4.20. The QE is much lower than that of the superlattice, but the lifetime was much longer; so given enough laser power, it was able to provide current from the same location for several weeks.

Even though the superlattice cathode has a small analyzing power, the QE hole and stray electrons from the unanodized edges of the cathode surface caused very large and unstable beam properties in the hall. The measured helicity-correlated beam asymmetries for 2004 are plotted versus slug in Figure 4.21. The position differences for three of the target BPMs significantly decreased in magnitude and were more stable with the Gun 2 source setup. The energy differences were of the same order, probably due to beam loading, while the jitter decreased. The charge asymmetry was essentially unchanged although Hall C feedback issues significantly contributed to the non-zero $A_{Q}$ during the second half of the experiment.

In 2005, an anodized superlattice cathode was used for the entire run of HAPPEX- ${ }^{4} \mathrm{He}$ and HAPPEX-H which ran for $\sim 15$ weeks and maintained high polarization and high QE with stable position differences over the entire period, as shown in Figure 4.22. 

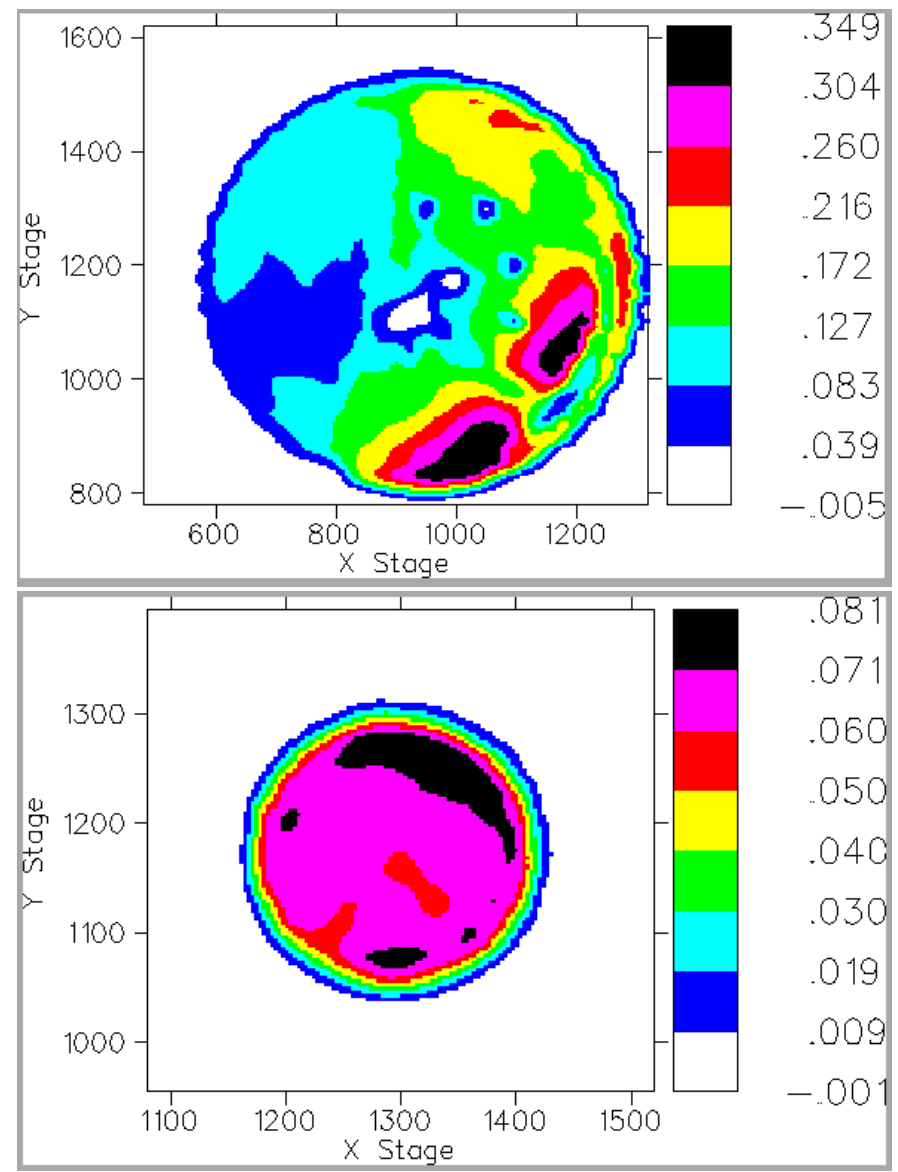

Figure 4.20. Top: Superlattice cathode QE after use for the 2004 HAPPEX production running. The large white spot in the center corresponds to the electrostatic center of the cathode where a large QE hole was created. The smaller white spot and dark-blue spots surrounding it are the different locations used for production running. Bottom: Strainedlayer cathode QE after the 2004 HAPPEX production run. The colored-scale on the right corresponds to the cathode QE in percent. The $x$ and $y$ scales are arbitrary units with 300 units $\simeq 5 \mathrm{~mm}$. 

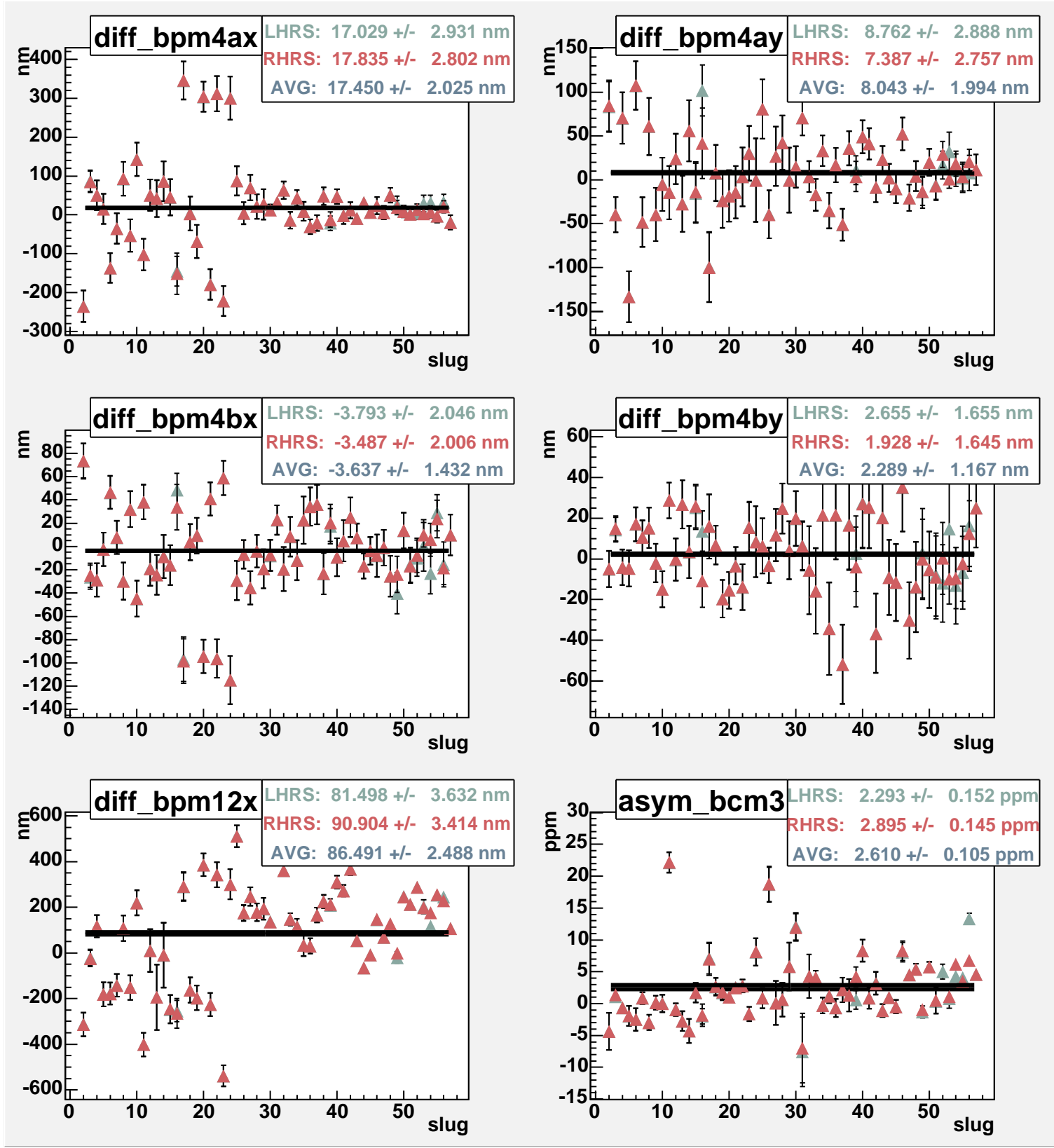

Figure 4.21. The 2004 helicity-correlated beam asymmetries versus slug plotted for the LHRS (green) and RHRS (red). The average values for each HRS and the global average (blue) are shown in the box in the upper right corner of each plot. 

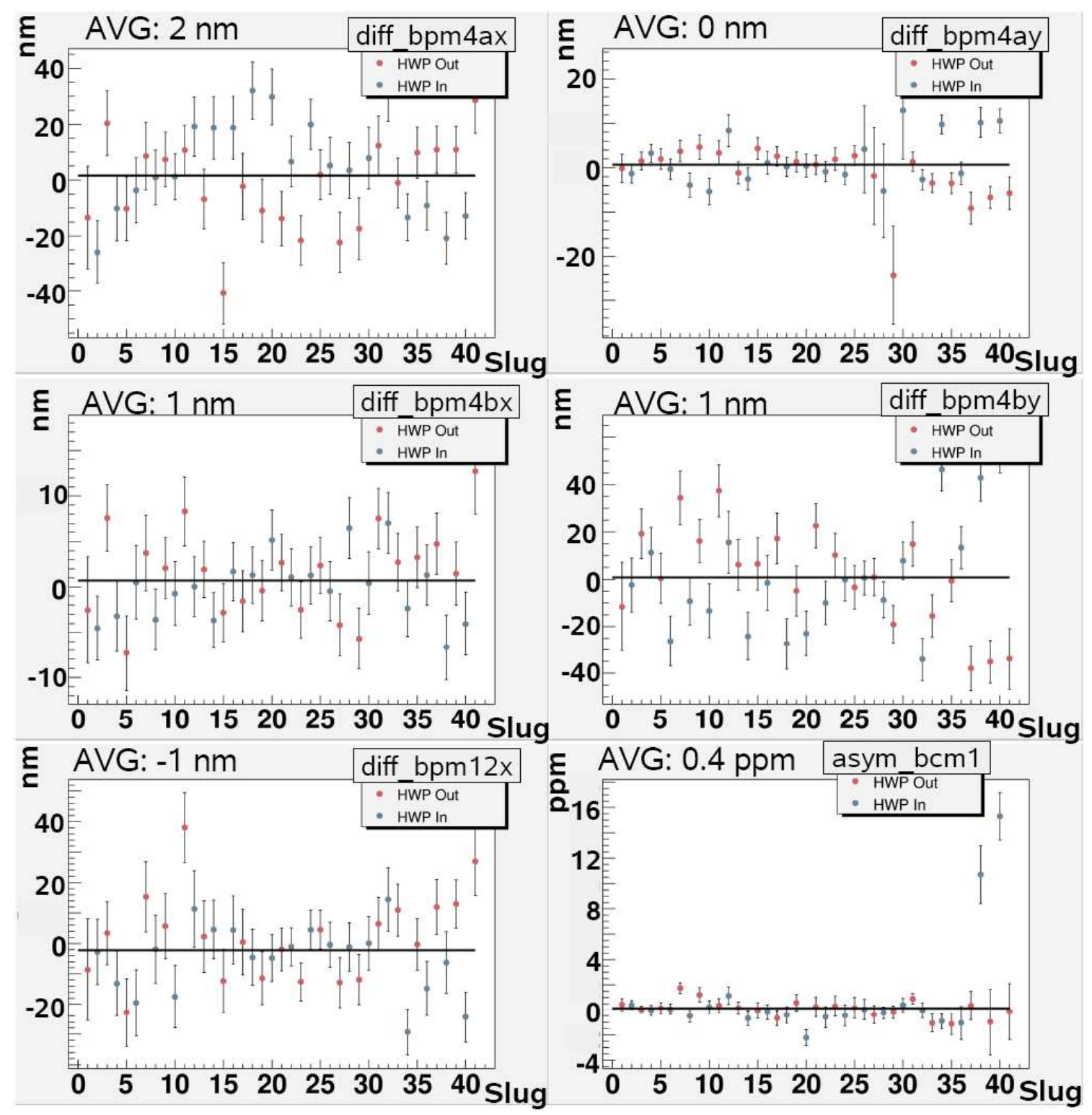

Figure 4.22. The 2005 helicity-correlated beam asymmetries versus slug plotted for the IHWP "in" (blue) and "out" (red) states. The average values are shown in the upper left corner of each plot. 


\section{CHAPTER 5 ASYMMETRY ANALYSIS}

The HAPPEX-H data was accumulated in two significant data-taking periods, JuneJuly 2004 and October-November 2005. This chapter includes a detailed description of the raw asymmetry analysis and the corrections to the asymmetry. The physics asymmetry is extracted after also conducting several background and normalization analyses which will be described briefly. The final results from 2004 are published in [45] and the 2005 results have been accepted for publication in Physical Review Letters [46].

\subsection{Overview}

The parity-violating asymmetry, also referred to as the physics asymmetry is extracted from the data in the following way:

$$
A_{P V}=\frac{K}{P_{b}} \frac{\left(A_{\text {raw }}-F-T\right)-P_{b} \sum_{i} A_{i} f_{i}}{1-\sum_{i} f_{i}}
$$

where $P_{b}$ is the beam polarization, $A_{\text {raw }}$ is the normalized detector asymmetry, $F$ is the false beam asymmetry correction, $T$ is the transverse beam asymmetry correction, $f_{i}$ are background fractions and $A_{i}$ are the associated background asymmetries, and $K$ accounts for the finite kinematic acceptance.

The raw asymmetry analysis includes the determination of the $\left(A_{\text {raw }}-F-T\right)$ piece of $A_{P V}$ which will be discussed in the following section. The determination of the remaining terms and factors of $A_{P V}$ which account for the background and normalization corrections will be discussed briefly in Sections 5.3-5.6.

We performed a "blinded" analysis where the asymmetry has an offset applied to it which is significantly larger than the statistical error of the asymmetry and is only removed once all the analysis tasks are completed. 


\subsection{Raw Asymmetry Analysis}

The determination of the corrected raw asymmetry consists of several steps:

1. Make data-quality cuts

2. Calculate the raw asymmetry

3. Calculate the helicity-correlated beam asymmetries

4. Calculate the detector sensitivities to the helicity-correlated beam asymmetries

5. Make corrections to the raw asymmetry for the false beam asymmetries

6. Check that the data is statistically well-behaved.

The rest of this section will discuss these steps in more detail.

\subsubsection{Data-Quality Cuts}

There are three types of cuts on the data, two of which are built into our analysis software, PAN. None of the data-quality cuts are helicity-dependent. PAN has the ability to flag the event that fails the cut conditions and defines a cut interval around the event. The cut interval is used to avoid creating a helicity-biased cut in the data.

The first type of cut uses PAN to check the status of the signals in the DAQ. We define a cut to verify that the helicity sequence is correct by checking that the two helicity windows in each pair are of opposite helicity. If a window does not satisfy this constraint, it is flagged as "bad" and so are the 25 windows before and after the event. Additionally, PAN also checks that the detectors and beam monitor signals are not saturating the ADCs. Events with ADC signals over 60000 channels are flagged along with the 5 windows before and 10 after. Also, an absolute cut on the beam intensity was used to ensure that all runs were taken with similar detector rates, and therefore, similar statistical widths. The allowed current range was $>20 \mu \mathrm{A}$ in 2004 and $>45 \mu \mathrm{A}$ in 2005 .

The second type of cut uses PAN to remove periods of instability in the beam intensity, position, angle, and energy by making comparisons between adjacent helicity windows. The main concern is that large excursions in the beam parameters can cause non-linear responses 


\begin{tabular}{|l|c|c|c|c|c|c|}
\hline & \multicolumn{3}{|c|}{2004} & \multicolumn{3}{c|}{2005} \\
\hline \hline Cut & Threshold & Extent lo & Extent hi & Threshold & Extent lo & Extent hi \\
\hline BCM1 & - & - & - & $3 \mu \mathrm{A}$ & 10 & 40 \\
BCM3 & $0.6-1 \mu \mathrm{A}$ & 30 & 30 & - & - & - \\
BPM4A $x$ & $200 \mu \mathrm{m}$ & 10 & 100 & $100 \mu \mathrm{m}$ & 10 & 10 \\
BPM4B $x$ & $100 \mu \mathrm{m}$ & 10 & 100 & $30 \mu \mathrm{m}$ & 10 & 10 \\
BPM4A $y$ & $100 \mu \mathrm{m}$ & 10 & 100 & $40 \mu \mathrm{m}$ & 10 & 10 \\
BPM4By & $100 \mu \mathrm{m}$ & 10 & 100 & $100 \mu \mathrm{m}$ & 10 & 10 \\
BPM12x & $250 \mu \mathrm{m}$ & 10 & 100 & $100 \mu \mathrm{m}$ & 10 & 100 \\
\hline
\end{tabular}

Table 5.1. A summary of the 2004 and 2005 cuts and cut intervals to remove periods of beam instability. The cuts are for changes in the beam parameters greater than the values listed, and the intervals are defined by the number of windows before (lo) and after (hi) the "bad" event.

in the detector. The thresholds and intervals for these cuts are determined empirically by studying the size of the instability in the beam parameters and the time it takes for the monitoring devices and detector to recover from these instabilities. Then these limits and intervals are set in PAN for a reanalysis of the data. A beam intensity cut was used so that the current in each run did not vary more than $\sim 5 \%$. In 2004 , the position and energy thresholds were set to remove beam instabilities greater than eight times the natural $30 \mathrm{~Hz}$ noise, or "jitter," of the beam parameter. In 2005, the thresholds were set to remove instabilities greater than 14 times the jitter. The main difference between the 2004 and 2005 thresholds is due to the fact that the 2005 beam tune created much less beam jitter at each BPM location than in 2004. The beam quality cuts and cut intervals used in 2004 and 2005 are summarized in Table 5.1.

The third type of cut manually sets event cuts to remove periods when the hardware was not working properly. We remove runs or periods of runs where any of the spectrometer magnets are broken, a detector is broken, or the beamline monitors are broken. If only the left or right HRS or detector is broken, we keep the data from the working HRS. In 2004, we also cut out the beam modulation cycles from the final data set.

The final data sets consist of $\sim 11 \times 10^{6}$ helicity window pairs for 2004 and $\sim 26.4 \times 10^{6}$ helicity window pairs for 2005. 


\subsubsection{Raw Asymmetry}

The detector asymmetry is calculated for each detector segment for each window pair as:

$$
A_{\mathrm{det}}=\frac{D^{R}-D^{L}}{D^{R}+D^{L}}
$$

but the raw asymmetry is calculated in the same way using the normalized detector flux defined by:

$$
A_{\text {raw }}=\frac{D^{R} / I^{R}-D^{L} / I^{L}}{D^{R} / I^{R}+D^{L} / I^{L}}
$$

where $D^{R}\left(D^{L}\right)$ and $I^{R}\left(I^{L}\right)$ are the integrated detector signal and integrated current signal respectively for the right (left) helicity window. For every data run, each pulsepair asymmetry calculated is histogrammed, and the arithmetic mean is calculated for the average asymmetry of the run. The raw asymmetry is calculated for each detector and for left (Det_L), right (Det_R), and all (Det_All) detector combinations for each run.

The asymmetry for each detector combination is calculated as an asymmetry of the average detector rather than an average of the individual detector asymmetries in order to properly account for correlations between the detectors caused by target density fluctuations. The proper weights for averaging the detector signals must take into account the differences in PMT gain and rate in the individual detectors. The normalized detector signal for a single helicity window is defined as $S_{k}=D_{k} / I$ where the subscript $k$ specifies the detector segment. When taking the average of the detectors, we normalize $S_{k}$ to the average detector signal, $\left\langle S_{k}\right\rangle$, in order to take into account differences in the PMT gain which affect the raw signal size. When calculating the average, we also want to take into account the statistical weight of each detector based on its rate. The width of the pulse-pair asymmetry distribution for a detector is $\sigma_{A_{k}}=1 / \sqrt{N_{k}}$ where $N_{k}$ is the number of electrons incident on the $k$ th detector for a helicity window pair; therefore, the statistical weight for the detector signal becomes $1 / \sigma_{A_{k}}^{2}$ (because there is no significant source of nonstatistical fluctuations). The resulting weight for each detector segment is:

$$
w_{k}=\frac{1}{\left\langle S_{k}\right\rangle \sigma_{A_{k}}^{2}}
$$


In order to simplify the calculation of the average asymmetry for each detector combination, we normalize the weights to one by:

$$
w_{k}^{\prime}=\frac{w_{k}}{w_{1}+w_{2}+w_{3}+w_{4}} .
$$

Then the raw asymmetry of a helicity window pair for the detector combinations is calculated as follows:

$$
\begin{aligned}
A_{\mathrm{raw}}^{\mathrm{Left}} & =\frac{\left(S_{1}^{R} w_{1}^{\prime}+S_{2}^{R} w_{2}^{\prime}\right)-\left(S_{1}^{L} w_{1}^{\prime}+S_{2}^{L} w_{2}^{\prime}\right)}{\left(S_{1}^{R} w_{1}^{\prime}+S_{2}^{R} w_{2}^{\prime}\right)+\left(S_{1}^{L} w_{1}^{\prime}+S_{2}^{L} w_{2}^{\prime}\right)}, \\
A_{\mathrm{raw}}^{\mathrm{Right}} & =\frac{\left(S_{3}^{R} w_{3}^{\prime}+S_{4}^{R} w_{4}^{\prime}\right)-\left(S_{3}^{L} w_{3}^{\prime}+S_{4}^{L} w_{4}^{\prime}\right)}{\left(S_{3}^{R} w_{3}^{\prime}+S_{4}^{R} w_{4}^{\prime}\right)+\left(S_{3}^{L} w_{3}^{\prime}+S_{4}^{L} w_{4}^{\prime}\right)},
\end{aligned}
$$

and

$$
A_{\mathrm{raw}}^{\mathrm{All}}=\frac{\left(S_{1}^{R} w_{1}^{\prime}+S_{2}^{R} w_{2}^{\prime}+S_{3}^{R} w_{3}^{\prime}+S_{4}^{R} w_{4}^{\prime}\right)-\left(S_{1}^{L} w_{1}^{\prime}+S_{2}^{L} w_{2}^{\prime}+S_{3}^{L} w_{3}^{\prime}+S_{4}^{L} w_{4}^{\prime}\right)}{\left(S_{1}^{R} w_{1}^{\prime}+S_{2}^{R} w_{2}^{\prime}+S_{3}^{R} w_{3}^{\prime}+S_{4}^{R} w_{4}^{\prime}\right)+\left(S_{1}^{L} w_{1}^{\prime}+S_{2}^{L} w_{2}^{\prime}+S_{3}^{L} w_{3}^{\prime}+S_{4}^{L} w_{4}^{\prime}\right)} .
$$

The individual detector segments are used to understand and calculate systematic errors, but the detector combinations are used to calculate the final asymmetry as discussed in Section 5.2.3.2. The raw pulse-pair asymmetry distributions for the 2004 detector combinations are shown in Figure 5.1, and the 2005 detector combinations are shown in Figure 5.2.

\subsubsection{False Asymmetry Corrections}

False asymmetries are defined as asymmetries contributing to the $A_{\text {raw }}$ distribution that are not due to parity-violating interactions. The primary sources of false asymmetries come from helicity-correlated differences in the beam parameters on the target because of the cross section's dependence on these parameters. Additionally, electronic pickup of the helicity signal could cause a false asymmetry in the DAQ signals. $F$ in Equation 5.1 is the correction to $A_{\text {raw }}$ for these asymmetries. The $T$ term in Equation 5.1 is the correction for a false asymmetry that can be created by the interaction of the vertical beam polarization with the target which will be discussed in Section 5.2.3.5. 


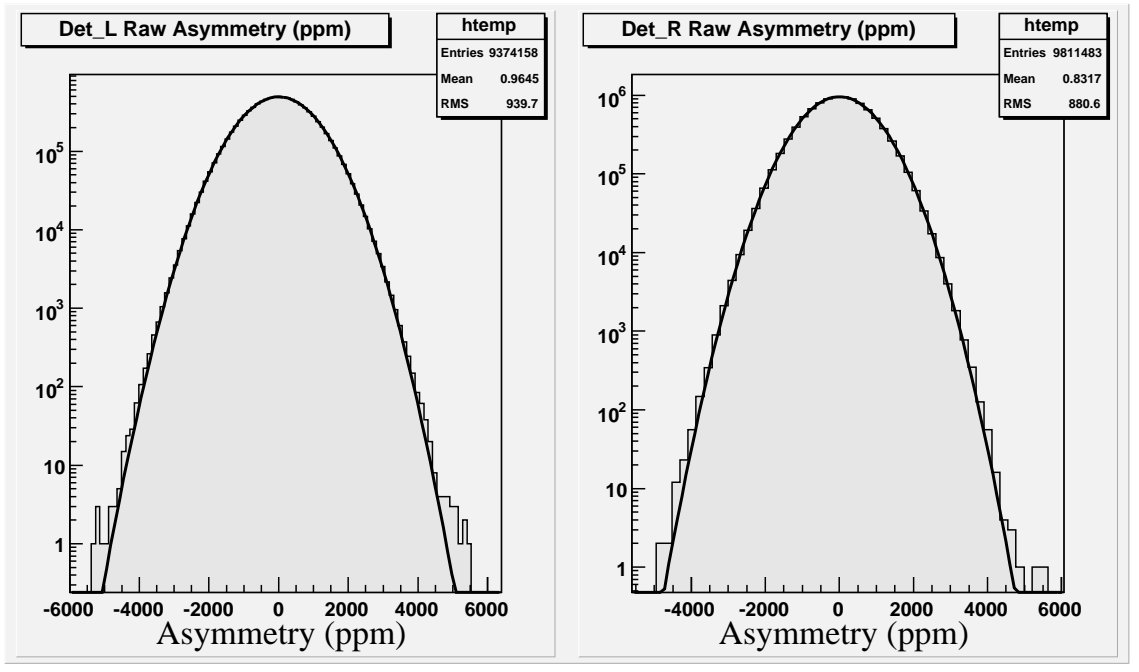

Figure 5.1. The $A_{\text {raw }}$ pulse-pair asymmetry distributions plotted on a vertical log scale for the 2004 left and right detector combinations. The distributions are Gaussian over five orders of magnitude.

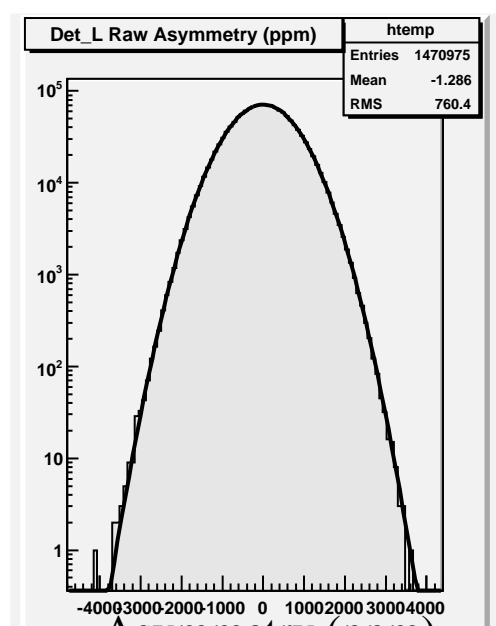

Asymmetry (ppm)
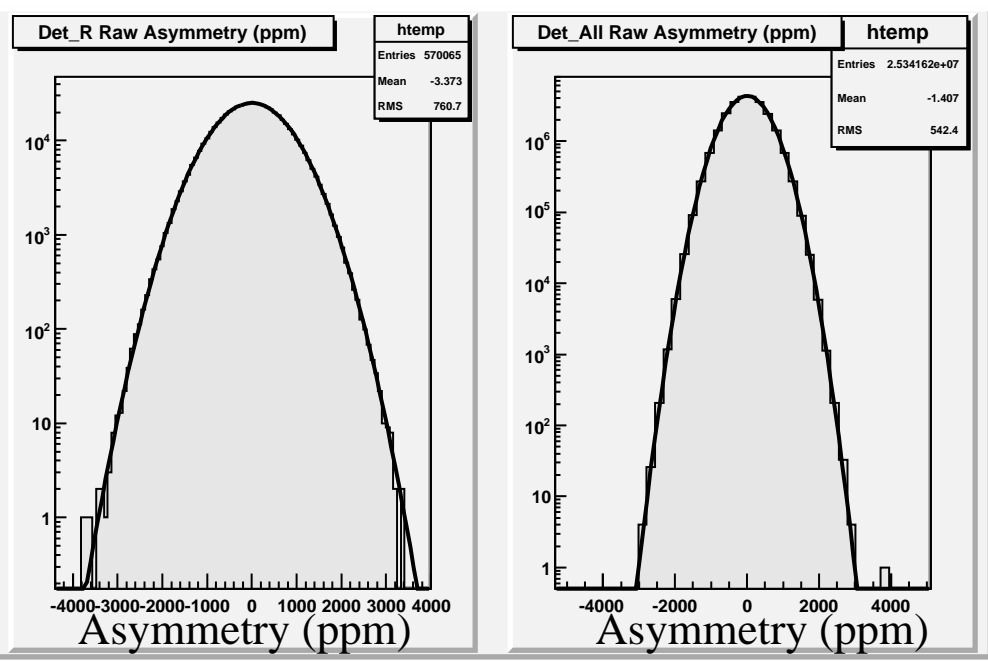

Figure 5.2. The $A_{\text {raw }}$ pulse-pair asymmetry distributions plotted on a vertical log scale for the 2005 left, right, and all detector combinations. The distributions are Gaussian over five orders of magnitude.

\subsubsection{Helicity-Correlated Beam Asymmetries}

The charge asymmetry and beam position differences are calculated as:

$$
A_{Q}=\frac{I^{R}-I^{L}}{I^{R}+I^{L}}, \quad \Delta x_{i}=x_{i}^{R}-x_{i}^{L}
$$


where the subscript $i$ denotes the BPM and $x_{i}^{R}\left(x_{i}^{L}\right)$ is the beam position measured for the right (left) helicity state for the $i$ th BPM. BCM3 was used for the current measurement in 2004, and BCM1 was used in 2005. There are five beam position measurements: 4Ax, 4Ay, $4 \mathrm{~B} x, 4 \mathrm{~B} y$, and $12 x$ which together determine the position, angle, and energy at the target.

The raw asymmetry is corrected for the helicity-correlated beam asymmetries by:

$$
A_{\text {corr }}=A_{\text {raw }}+\sum_{i=1}^{5} \beta_{i} \Delta x_{i}
$$

where $\beta_{i}$ is the normalized detector sensitivity to the beam position at the $i$ th BPM. No separate $A_{Q}$ correction to $A_{\text {raw }}$ is necessary here because we normalize the detector response to the beam current to calculate $A_{\text {raw }}$. $A_{Q}$ can still contribute to a false asymmetry and error in the correction due to a non-linearity in the detector response. This will be discussed in Section 5.2.3.4.

The $\Delta x_{i}$ are minimized according to the methods described in Section 4.2 to ensure that the corrections to the raw asymmetry are small. Plots of the beam position differences and $A_{Q}$ for the 2004 and 2005 runs are shown in Figures 4.21 and 4.22, and the averages over the entire run for 2004 and 2005 are summarized in Table 4.2. The average helicity-correlated beam asymmetries are calculated in the same way that the detector asymmetries are calculated; i.e. the $\Delta x_{i}$ contributing to the left detector are weighted by the left detector's statistical weight and the same is done to calculate the $\Delta x_{i}$ contributions to the other two detector combinations.

The passive helicity reversal provided by the IHWP provides not only a suppression of some sources of $\Delta x_{i}$, but it also provides a suppression of false asymmetries due to electronic crosstalk and serves as a crosscheck that the systematics associated with these false asymmetries are small. Because the DAQ and analysis software are unaware of the passive helicity flip, the sign of the measured asymmetry will change while the magnitude remains the same. The IHWP is inserted every few hours of data-taking, and then the data for each IHWP period is grouped together into "slugs." 


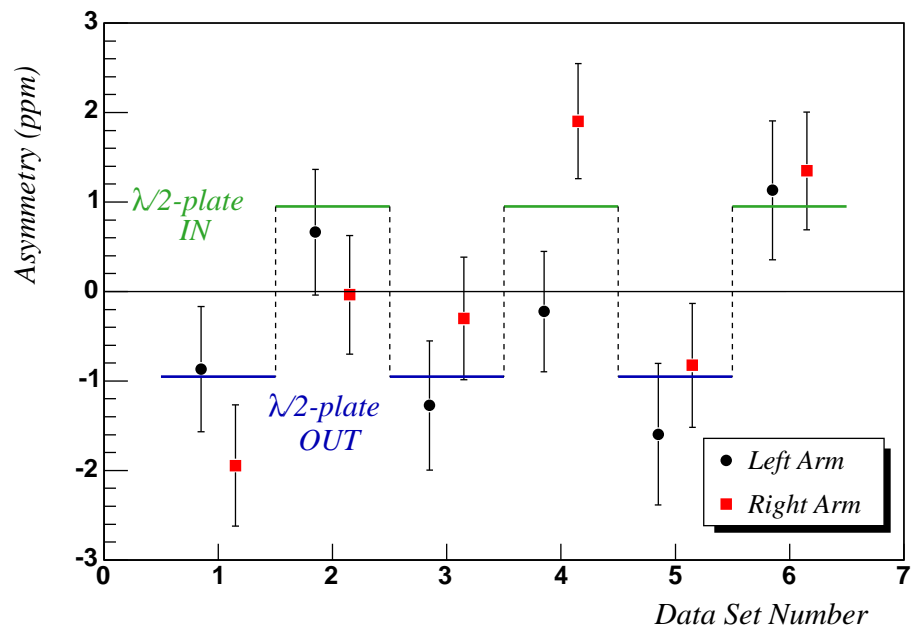

Figure 5.3. $A_{\text {corr }}$ for all 2004 data, grouped by $\lambda / 2$-plate state in 6 sequential data sets. The circles and squares represent the average of the 2 PMT channels in each spectrometer arm, and the line represents $A_{\text {corr }}$, averaged over the run and plotted with the appropriate sign for each $\lambda / 2$-plate state.

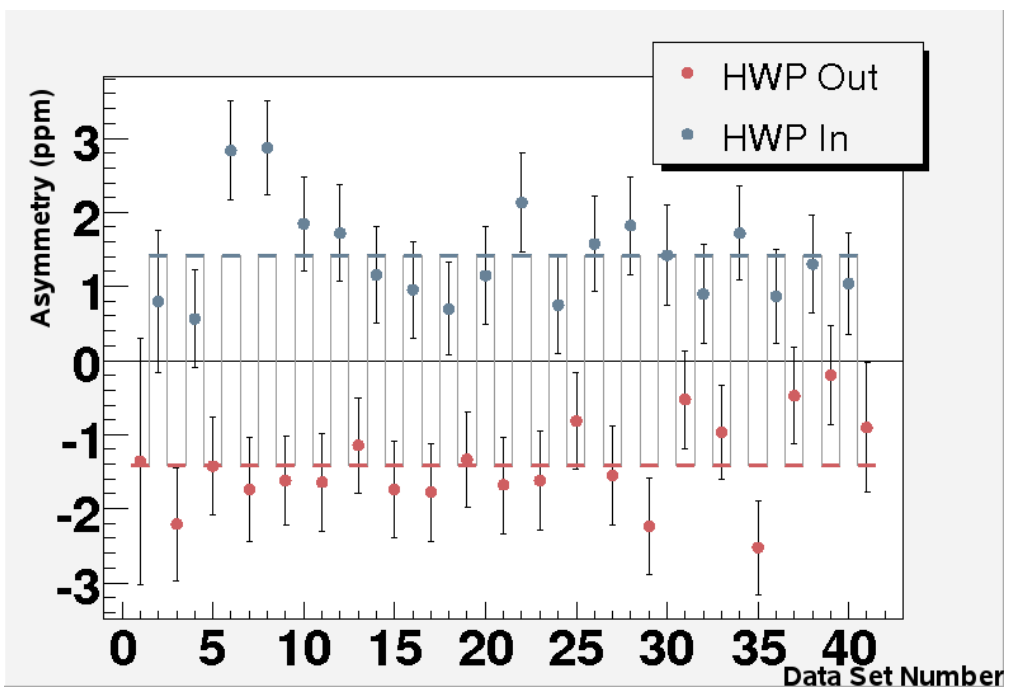

Figure 5.4. $A_{\text {corr }}$ for all 2005 data, grouped by $\lambda / 2$-plate state for sequential data sets. The line represents $A_{\text {corr }}$, averaged over the run and plotted with the appropriate sign for each $\lambda / 2$-plate state.

The correlation of $A_{\text {corr }}$ with IHWP is shown in Figure 5.3 for 2004 and Figure 5.4 for 2005. Observed fluctuations are consistent with purely statistical fluctuations with a $\chi^{2}$ per degree of freedom of 1.0 for 2004 and 0.89 for 2005. 
The measurement of the $\Delta x_{i}$ is not sufficient to make a correction to the raw asymmetry for these effects. We must also measure the detector sensitivities to $x_{i}$. The $\beta_{i}$ from Equation 5.8 are determined using linear regression and beam modulation methods. In order to make sure the corrections are applied to the data correctly, the results for both methods are compared. The beam modulation method is used to obtain the final results. Details regarding the regression analysis are discussed in [22], and the beam modulation analysis is briefly described in the next section.

\subsubsection{Beam Modulation}

Beam modulation makes deliberate changes to the beam position, angle, and energy in a helicity-uncorrelated way in order to understand the detector sensitivities to these parameters. During a beam modulation cycle, we measure the responses $\partial \sigma / \partial C_{j}$ and $\partial M_{i} / \partial C_{j}$ of the normalized detector flux, $\sigma$, and the beam position at the $i$ th BPM, $M_{i}$, to the $j$ th modulation coil, $C_{j}$.

In order to extract the normalized detector sensitivities to beam position, $\beta_{i}=\partial \sigma / \partial M_{i}$, we use the relationship:

$$
\frac{\partial \sigma}{\partial C_{j}}=\sum_{i=1}^{5}\left(\frac{\partial \sigma}{\partial M_{i}}\right)\left(\frac{\partial M_{i}}{\partial C_{j}}\right) .
$$

In order to solve this equation for $\partial \sigma / \partial M_{i}$, we must invert the $\partial M_{i} / \partial C_{j}$ matrix. In order to invert this matrix, the beam optics must be tuned such that the coil modulations span the position and angle space to satisfy that the matrix not be singular. Data from a representative beam modulation cycle is shown in Figure 5.5. A more general description of the beam modulation analysis which was used for HAPPEX can be found in $[23,24]$.

Using the measured $\beta_{i}$ and $\Delta x_{i}$, the total correction to the $2004 A_{\text {raw }}$ is -79 parts per billion (ppb), and the total correction to the $2005 A_{\text {raw }}$ is -10 ppb. The correction was much smaller in 2005 because the $\Delta x_{i}$ were an order of magnitude smaller in 2005 compared to 2004 .

The corrected pulse-pair asymmetry plots show that the asymmetry distributions are Gaussian over five orders of magnitude for both 2004 (Figure 5.6) and 2005 (Figure 5.7) data sets. The RMS widths are $624 \mathrm{ppm}$ and $540 \mathrm{ppm}$ for 2004 and 2005, respectively, on the 

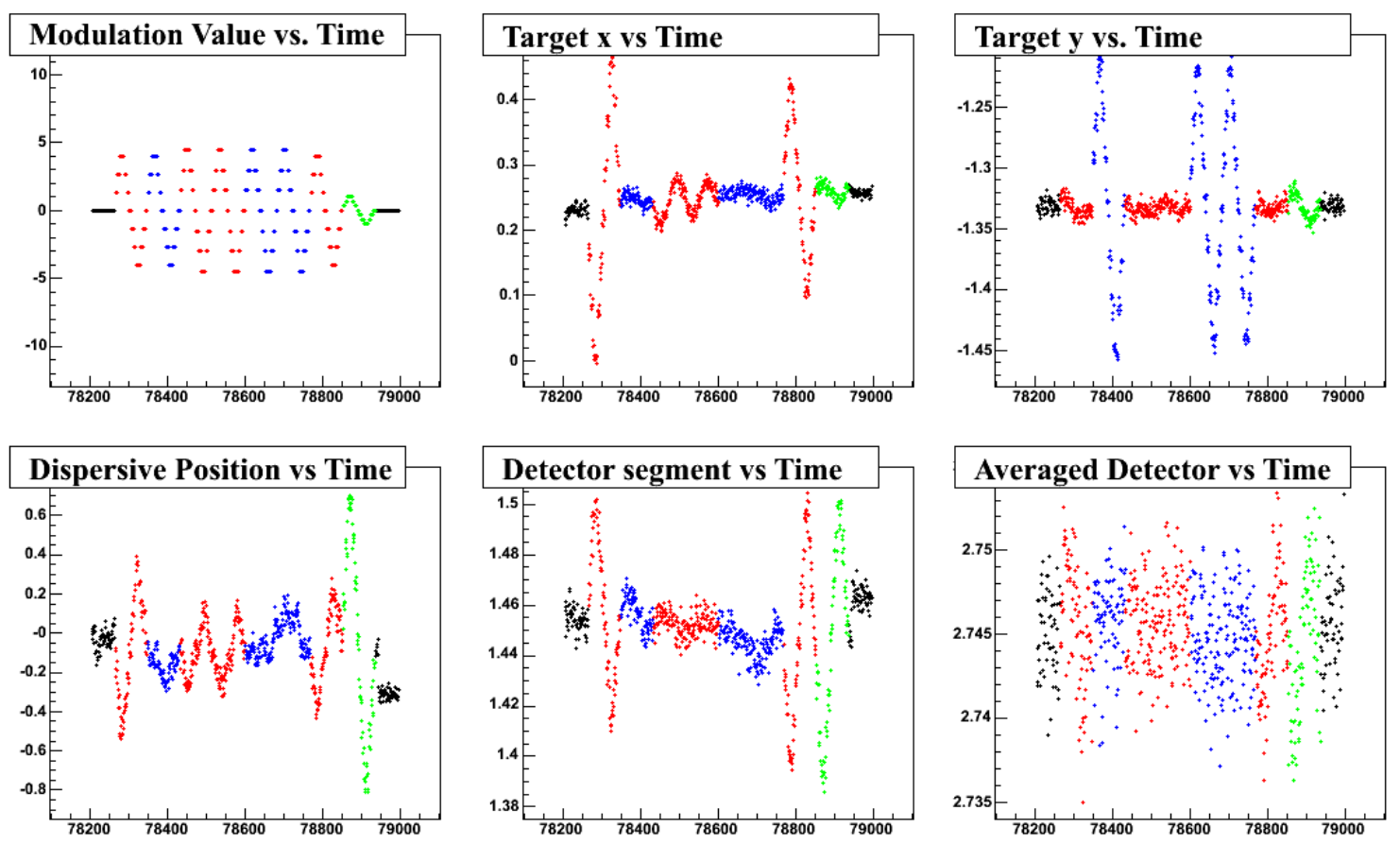

Figure 5.5. A beam modulation cycle: the red points are data from $x$ modulation, blue is $y$ modulation, and green is energy modulation. The top left plot shows the modulation value for each coil versus event number and the following plots show the BPM and detector responses during the modulation cycle. A beam modulation cycle lasts $\sim 23 \mathrm{~s}$.

combined left and right asymmetry distributions where the dominant source of fluctuations in the distribution is due to counting statistics.

The systematic error in the correction to $A_{\text {raw }}$ is determined by studying the statistical behavior of the individual detector asymmetries and their correlations to the helicitycorrelated beam asymmetries. The individual detector segments are 5-20 times more sensitive than the detector combinations to the helicity-correlated beam asymmetries because the elastic peak is divided between the detector segments. The measured sensitivities (beam modulation slopes) were divided into seven subsets for the 2005 data-taking period. The average slopes for each detector and BPM combination are summarized in Table 5.2.

The statistical behavior of the average corrected asymmetry for each detector is checked by calculating the pull $P_{n}$ for run $n$, defined as:

$$
P_{n}=\frac{\left\langle A_{\text {corr }}\right\rangle_{n}-\left\langle A_{\text {corr }}\right\rangle}{\sigma_{n}^{\text {corr }}}
$$



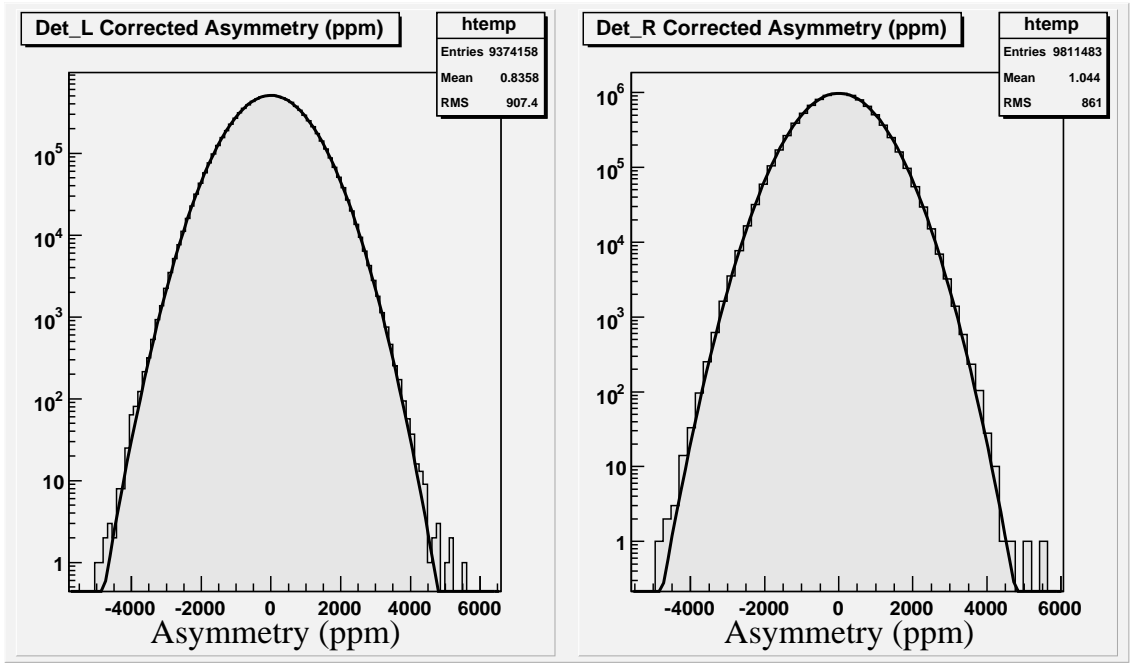

Figure 5.6. The $A_{\text {corr }}$ pulse-pair asymmetry distributions plotted on a vertical log scale for the 2004 left and right detector combinations.
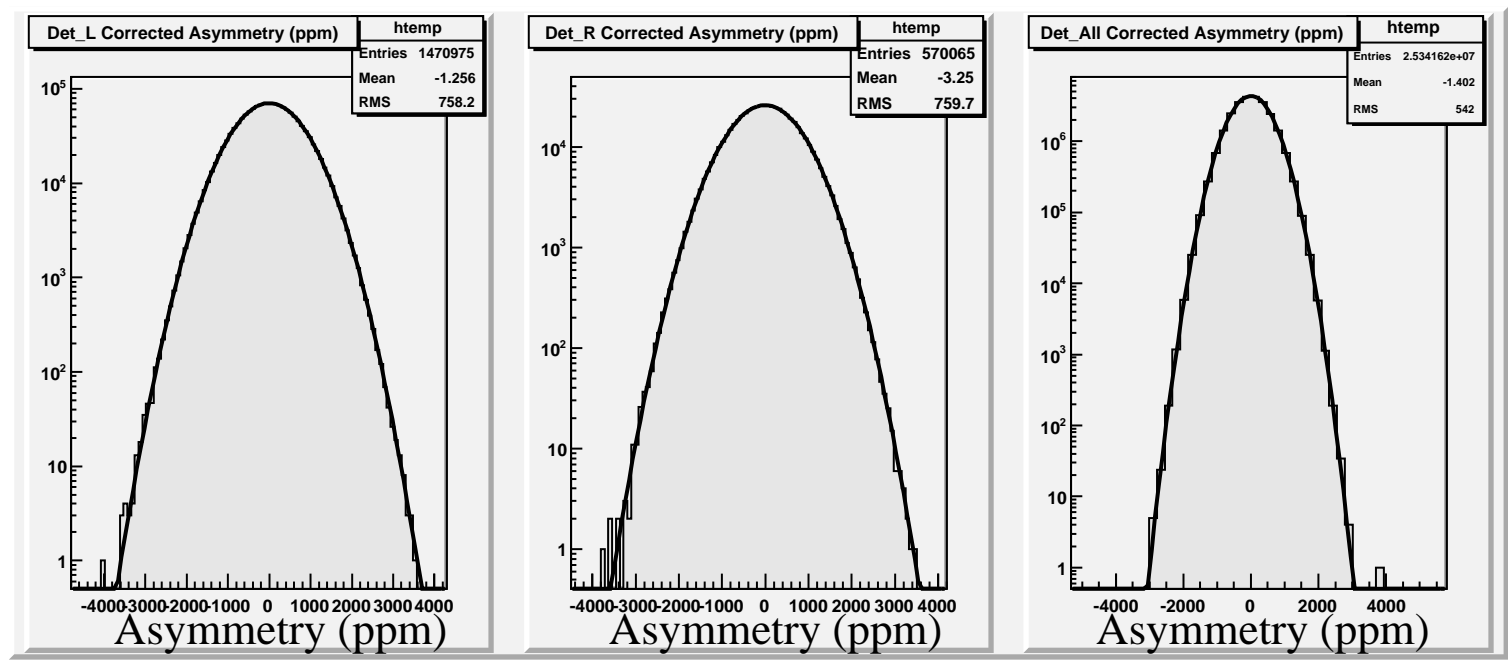

Figure 5.7. The $A_{\text {corr }}$ pulse-pair asymmetry distributions plotted on a vertical log scale for the 2005 left, right, and all detector combinations.

where $\left\langle A_{\text {corr }}\right\rangle_{n}$ is the average corrected asymmetry for run $n,\left\langle A_{\text {corr }}\right\rangle$ is the total average corrected asymmetry, and $\sigma_{n}^{\text {corr }}$ is the error on $\left\langle A_{\text {corr }}\right\rangle_{n}$. The measured asymmetry is statistically well-behaved for pull distributions that are Gaussian with unit variance, as shown in Figures 5.8 and 5.9. 

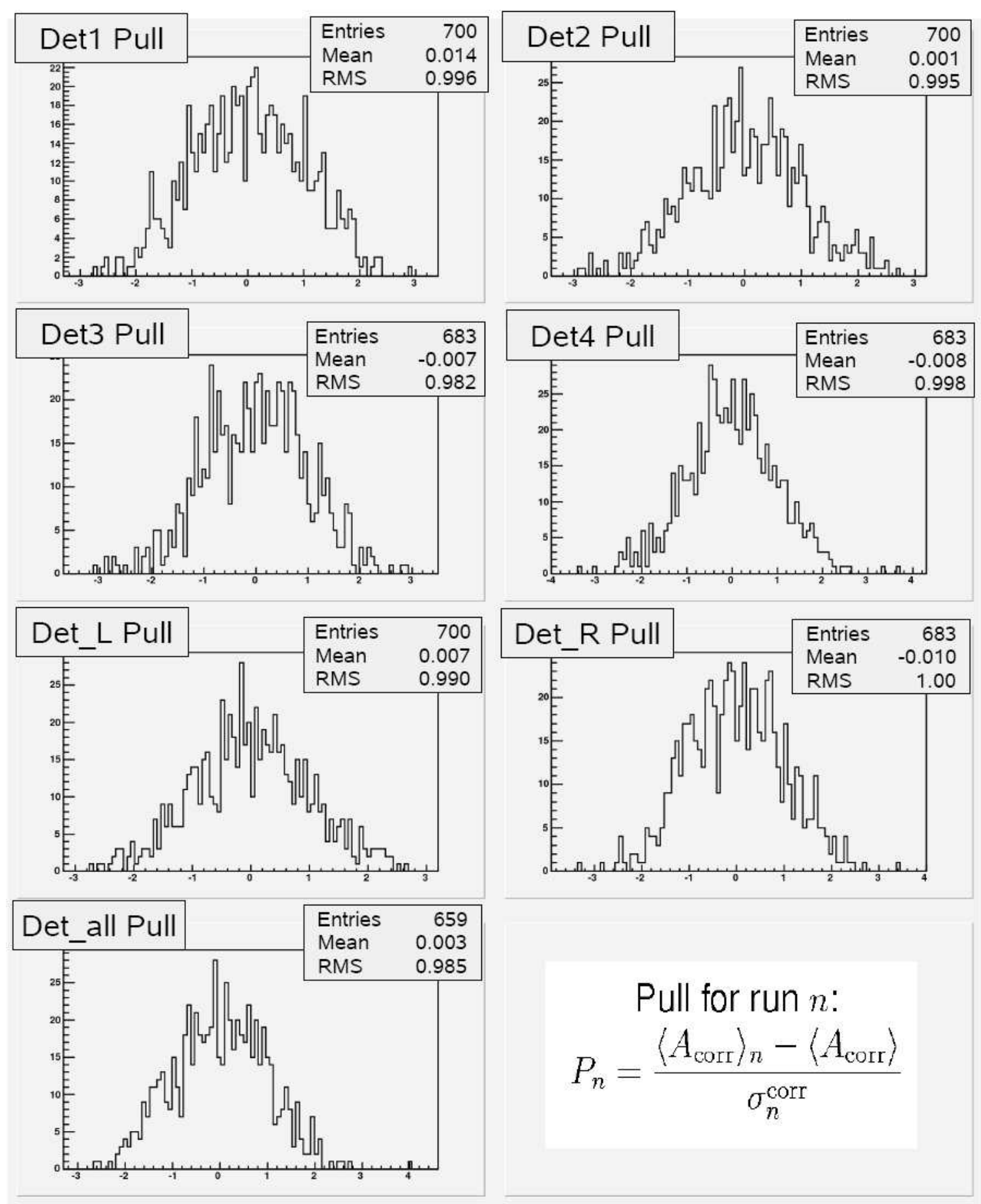

$$
\begin{gathered}
\text { Pull for run } n: \\
P_{n}=\frac{\left\langle A_{\text {corr }}\right\rangle_{n}-\left\langle A_{\text {corr }}\right\rangle}{\sigma_{n}^{\text {corr }}}
\end{gathered}
$$

Figure 5.8. $2005 A_{\text {corr }}$ pull plots for all detectors. The mean of each distribution is 0 with an RMS of 1 , as expected. 


\begin{tabular}{|l|r|r|r|r|r|}
\hline & 4bx & 4by & 4ax & 4ay & 12x \\
\hline \hline det1 & 61.8 & 32.5 & 38.7 & -13.6 & -45.4 \\
det2 & -39.5 & -5.0 & 0.7 & 2.6 & 11.7 \\
det3 & 92.7 & 9.1 & -16.8 & -3.4 & -14.6 \\
det4 & -67.8 & -8.8 & 1.6 & 4.0 & 17.3 \\
det_1 & -18.6 & 2.7 & 8.5 & -0.7 & -0.1 \\
det_r & 14.5 & 0.4 & -7.8 & 0.2 & 1.0 \\
det_all & 3.5 & 1.2 & -2.4 & -0.2 & 0.6 \\
\hline
\end{tabular}

Table 5.2. 2005 average beam modulation slopes for each detector in units of $\mathrm{ppm} / \mu \mathrm{m}$.
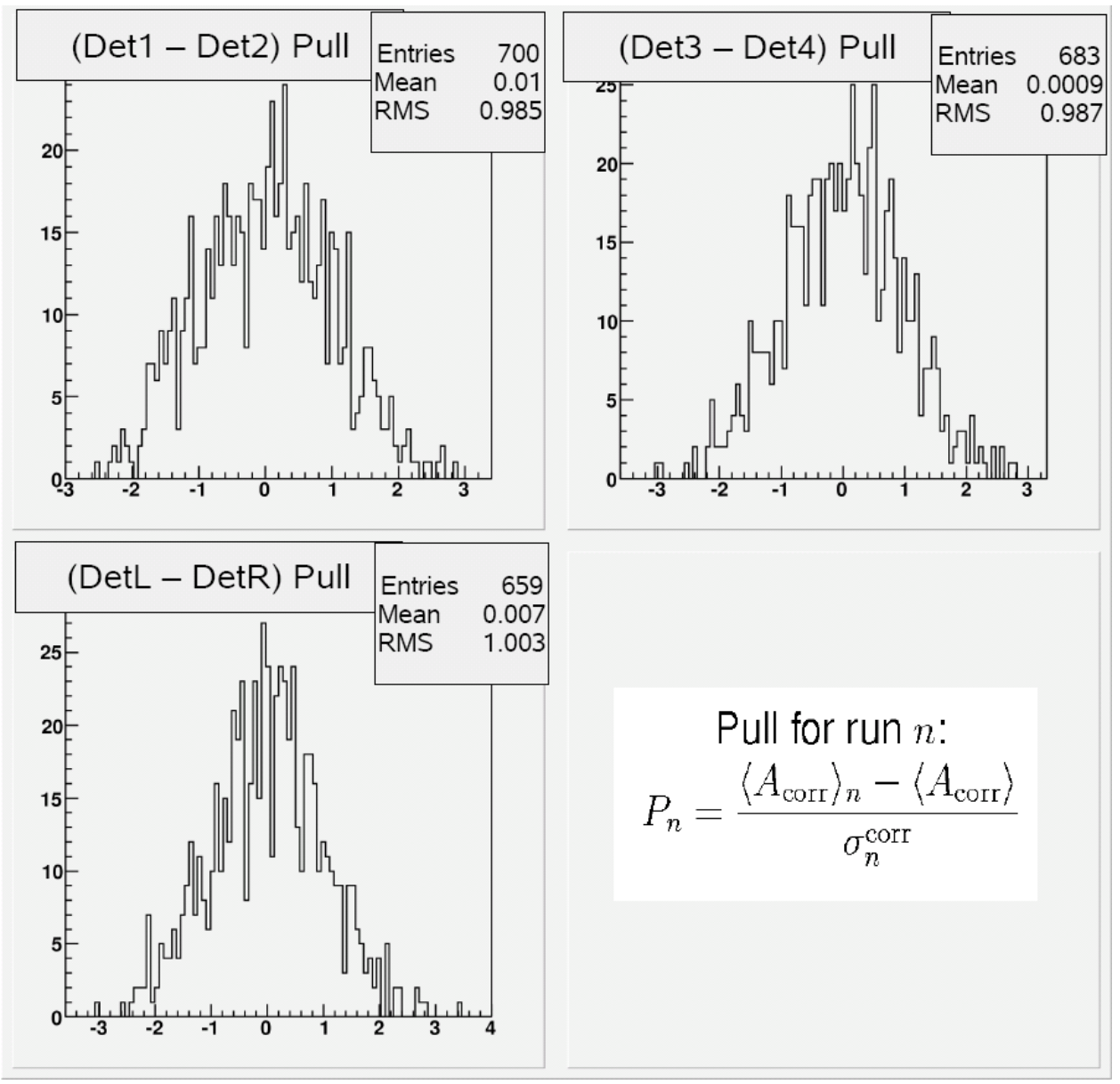

Figure 5.9. $2005 A_{\text {corr }}$ pull plots for detector differences. The mean of each distribution is 0 with an RMS of 1 , as expected. 


\begin{tabular}{|l|r|r|r|r|r|r|}
\hline & 4bx & 4by & 4ax & 4ay & 12x & Total \\
\hline \hline$\Delta$ (nm) (LHRS) & 1 & 1 & 2 & 0 & -1 & \\
\hline \hline$\Delta$ (nm) (RHRS) & 1 & 1 & 2 & 0 & -1 & \\
\hline \hline det1 & 0.05 & 0.05 & 0.06 & -0.00 & 0.05 & 0.20 \\
det2 & -0.03 & -0.01 & 0.00 & 0.00 & -0.01 & -0.05 \\
det3 & 0.08 & 0.01 & -0.04 & -0.00 & 0.01 & 0.07 \\
det4 & -0.06 & -0.01 & 0.00 & 0.00 & -0.01 & -0.08 \\
det_1 & -0.01 & 0.01 & 0.02 & -0.00 & 0.00 & 0.01 \\
det_r & 0.02 & 0.00 & -0.02 & 0.00 & 0.00 & 0.01 \\
det_all & 0.01 & 0.00 & 0.00 & -0.00 & 0.00 & 0.01 \\
\hline
\end{tabular}

Table 5.3. 2005 measured position differences for the entire run and corresponding corrections to $A_{\text {raw }}(\mathrm{ppm})$ for each detector and detector combination.

The 2005 position differences for each BPM and the correction for each detector is shown in Table 5.3. Det1 is the most sensitive detector, $\sim 20$ times more than any of the detector combinations, and the largest corrections are due to the $x$ position differences. The raw asymmetry compared to the corrected asymmetry for each detector and the differences of detectors is summarized in Table 5.4. The grand averages for the position differences are small $(<2 \mathrm{~nm})$, and the asymmetry central values and errors are virtually the same for the raw and corrected asymmetry such that there is very little systematic error (if any) due to the correction. Because of the good statistical behavior of the individual detector asymmetries and the detector differences, we conclude that the energy differences are well measured and understood. For this reason and because the correction for energy differences is negligible, we do not assign a systematic error. The dominant corrections are from BPM4ax and BPM4bx for which we assign a 100\% systematic error of $10 \mathrm{ppb}$ for each. The resulting systematic error assigned to the $A_{\text {raw }}$ correction is 14 ppb for 2005 .

In 2004, a similar process of comparing the asymmetry and helicity-correlated asymmetries for the individual detectors and detector differences was carried out. The systematic error for the correction due to energy differences was $12 \mathrm{ppb}$, and the error for the position and angle correction was $30 \mathrm{ppb}$. The resulting systematic error assigned to the $A_{\text {raw }}$ correction was 32 ppb for 2004. 


\begin{tabular}{|l|r|r|c|}
\hline & Raw & Beam Mod & Raw - Beam Mod \\
\hline \hline det1 & $-0.624 \pm 0.303$ & $-0.811 \pm 0.295$ & 0.187 \\
det2 & $-1.595 \pm 0.166$ & $-1.547 \pm 0.165$ & -0.048 \\
det3 & $-1.061 \pm 0.192$ & $-1.124 \pm 0.192$ & 0.063 \\
det4 & $-2.019 \pm 0.228$ & $-1.940 \pm 0.228$ & -0.078 \\
det_l & $-1.359 \pm 0.147$ & $-1.371 \pm 0.146$ & 0.012 \\
det_r & $-1.459 \pm 0.149$ & $-1.463 \pm 0.148$ & 0.005 \\
det_all & $-1.391 \pm 0.107$ & $-1.403 \pm 0.107$ & 0.012 \\
det1-det2 & $0.967 \pm 0.345$ & $0.731 \pm 0.338$ & 0.236 \\
det3-det4 & $0.964 \pm 0.298$ & $0.821 \pm 0.298$ & 0.143 \\
det_l-det_r & $0.035 \pm 0.212$ & $0.029 \pm 0.212$ & 0.006 \\
\hline
\end{tabular}

Table 5.4. $2005 A_{\text {raw }}(\mathrm{ppm})$ and $A_{\text {corr }}(\mathrm{ppm})$ comparison for each detector combination and detector differences for determination of systematic errors due to helicity-correlated corrections.

\section{Asymmetry Averaging Technique}

The final $A_{\text {corr }}$ was calculated differently for 2004 and 2005. The 2004 average is calculated as a $Q^{2}$-weighted, detector-weighted average between the left and right detector combinations because of the difference in the measured $Q^{2}$ for the two spectrometers. The 2005 average is calculated as a weighted average of the all detector combination with the left and right detector combinations for the periods of time where only one spectrometer was working.

The 2004 average corrected asymmetry is calculated in the following way. The runaveraged corrected left and right detector asymmetries are calculated as:

$$
\left\langle A_{\text {corr }}^{\text {Left }}\right\rangle=\frac{\sum_{n}\left\langle A_{\text {corr }}^{\text {Left }}\right\rangle_{n} /\left(\sigma_{n}^{\text {Left }}\right)^{2}}{\sum_{n} 1 /\left(\sigma_{n}^{\text {Left }}\right)^{2}} \text { and }\left\langle A_{\text {corr }}^{\text {Right }}\right\rangle=\frac{\sum_{n}\left\langle A_{\text {corr }}^{\text {Right }}\right\rangle_{n} /\left(\sigma_{n}^{\text {Right }}\right)^{2}}{\sum_{n} 1 /\left(\sigma_{n}^{\text {Right }}\right)^{2}}
$$

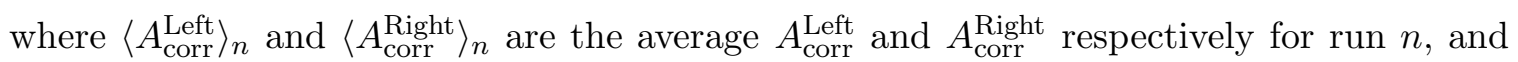
$\sigma_{n}^{\text {Left }}$ and $\sigma_{n}^{\text {Right }}$ are the errors on $\left\langle A_{\text {corr }}^{\text {Left }}\right\rangle_{n}$ and $\left\langle A_{\text {corr }}^{\text {Right }}\right\rangle_{n}$ respectively. From this, we then calculate an average corrected asymmetry normalized to $Q^{2}$ :

$$
\left\langle\frac{A_{\text {corr }}}{Q^{2}}\right\rangle=\frac{\left.\frac{\left\langle A_{\text {corr }}^{\text {Left }}\right\rangle}{\left\langle Q_{\text {Left }}^{2}\right\rangle} /\left(\frac{\sigma_{\text {cofr }}^{\text {Left }}}{\left\langle Q_{\text {Left }}^{2}\right.}\right\rangle\right)^{2}+\frac{\left\langle A_{\text {corr }}^{\text {Right }}\right\rangle}{\left\langle Q_{\text {Right }}^{2}\right\rangle} /\left(\frac{\sigma_{\text {corr }}^{\text {Right }}}{\left\langle Q_{\text {Right }}^{2}\right\rangle}\right)^{2}}{1 /\left(\frac{\sigma_{\text {cort }}^{\text {Lefr }}}{\left\langle Q_{\text {Left }}^{2}\right\rangle}\right)^{2}+1 /\left(\frac{\sigma_{\text {corr }}^{\text {Right }}}{\left\langle Q_{\text {Right }}^{2}\right\rangle}\right)^{2}}
$$


where $\left\langle Q_{\text {Left }}^{2}\right\rangle$ and $\left\langle Q_{\text {Right }}^{2}\right\rangle$ are the average $Q^{2}$ values for the LHRS and RHRS respectively.

The final corrected asymmetry for 2004 is calculated by:

$$
\left\langle A_{\text {corr }}\right\rangle=\left\langle\frac{A_{\text {corr }}}{Q^{2}}\right\rangle\left\langle Q^{2}\right\rangle
$$

where $\left\langle Q^{2}\right\rangle$ is the weighted average of $\left\langle Q_{\text {Left }}^{2}\right\rangle$ and $\left\langle Q_{\text {Right }}^{2}\right\rangle$ using the weights specified in Equation 5.12.

\section{Asymmetry Averaging Technique}

The 2005 corrected asymmetry is calculated using the all, left, and right detector combinations in the following way. The $2005 Q^{2}$ measured for each spectrometer was very similar, so a $Q^{2}$ weighted average was unnecessary to calculate. The 2005 data was taken with higher beam current; therefore, the statistical width of the asymmetry distributions was smaller and the target density fluctuations were slightly higher than in 2004. As a result, the all detector combination is used for the asymmetry average because it properly takes into account correlations between the detector segments. For the periods of time when only one spectrometer was working, the corresponding detector combination was used for the average. The number of helicity window pairs contained in each detector combination's asymmetry distribution is $25.3 \times 10^{6}$ pairs in the all combination, $1.5 \times 10^{6}$ pairs in the left combination, and $0.6 \times 10^{6}$ pairs in the right combination.

The corrected asymmetry is calculated for each run $n$ as:

$$
\left\langle A_{\mathrm{corr}}\right\rangle_{n}=\frac{\left\langle A_{\mathrm{corr}}^{\mathrm{All}}\right\rangle_{n} /\left(\sigma_{n}^{\mathrm{All}}\right)^{2}+\left\langle A_{\mathrm{corr}}^{\mathrm{L} ! \mathrm{R}}\right\rangle_{n} /\left(\sigma_{n}^{\mathrm{L} ! \mathrm{R}}\right)^{2}+\left\langle A_{\mathrm{corr}}^{\mathrm{R} ! \mathrm{L}}\right\rangle_{n} /\left(\sigma_{n}^{\mathrm{R} ! \mathrm{L}}\right)^{2}}{1 /\left(\sigma_{n}^{\mathrm{All}}\right)^{2}+1 /\left(\sigma_{n}^{\mathrm{L} ! \mathrm{R}}\right)^{2}+1 /\left(\sigma_{n}^{\mathrm{R} ! \mathrm{L}}\right)^{2}}
$$

where $\left\langle A_{\text {corr }}^{\text {All }}\right\rangle_{n}$ and $\sigma_{n}^{\text {All }}$ are the average corrected asymmetry of the all detector combination and error on the all average, respectively, for run $n,\left\langle A_{\text {corr }}^{\mathrm{L} ! \mathrm{R}}\right\rangle_{n}$ and $\sigma_{n}^{\mathrm{L} ! \mathrm{R}}$ are the average corrected asymmetry of the left detector combination when there is no right data and the error on that average for run $n$, and $\left\langle A_{\text {corr }}^{\mathrm{R} ! \mathrm{L}}\right\rangle_{n}$ and $\sigma_{n}^{\mathrm{R} ! \mathrm{L}}$ are the average corrected asymmetry 


\begin{tabular}{|c|c|c|c|}
\hline & IHWP OUT & IHWP IN & BOTH \\
\hline \hline \multicolumn{3}{|c|}{2004} \\
\hline$A_{\text {raw }}$ & $-0.99 \pm 0.32$ & $0.78 \pm 0.30$ & $-0.87 \pm 0.22$ \\
$A_{\text {corr }}$ & $-1.12 \pm 0.29$ & $0.80 \pm 0.29$ & $-0.95 \pm 0.20$ \\
\hline \hline \multicolumn{4}{|c|}{2005} \\
\hline$A_{\text {raw }}$ & $-1.40 \pm 0.15$ & $1.42 \pm 0.15$ & $-1.41 \pm 0.11$ \\
$A_{\text {corr }}$ & $-1.41 \pm 0.15$ & $1.43 \pm 0.15$ & $-1.42 \pm 0.11$ \\
\hline
\end{tabular}

Table 5.5. Raw and corrected asymmetries (in ppm) broken up by IHWP reversals. The differences between $A_{\text {raw }}$ and $A_{\text {corr }}$ result from corrections for energy, position, and angle differences.

of the right detector combination when there is no left data and the error on that average for run $n$. Then the final corrected asymmetry for 2005 is calculated by:

$$
\left\langle A_{\text {corr }}\right\rangle=\frac{\sum_{n}\left\langle A_{\text {corr }}\right\rangle_{n} /\left(\sigma_{n}^{\text {corr }}\right)^{2}}{\sum_{n} 1 /\left(\sigma_{n}^{\text {corr }}\right)^{2}} .
$$

The 2004 and 2005 raw and corrected asymmetries and corresponding statistical errors are summarized in Table 5.5. Under IHWP reversal, the absolute values of $A_{\text {corr }}$ are consistent within statistical errors. It is also worth noting that the corrections $\left(A_{\text {corr }}-A_{\text {raw }}\right)$ are much smaller than the corresponding error bars because the $\Delta x_{i}$ were well controlled during the experiment.

A final statistical check on the corrected asymmetry results is done by calculating the "pull" for the average corrected asymmetry for each run, see Equation 5.10. The histograms of the pulls for each detector are shown in Figure 5.8. The distributions are Gaussian with unit variance as expected.

The following sections (Sections 5.2.3.3, 5.2.3.4, and 5.2.3.5) discuss other sources of false asymmetries for which no correction was made because the size of the effect was negligible compared to the statistical error on the asymmetry.

\subsubsection{Helicity-Correlated Crosstalk}

Helicity-correlated crosstalk is defined as any electronic pickup of the helicity signal. In particular, we are concerned with a false asymmetry caused by electronic pickup of the helicity signal by the DAQ signals. In order to minimize the chances for electronic pickup, 


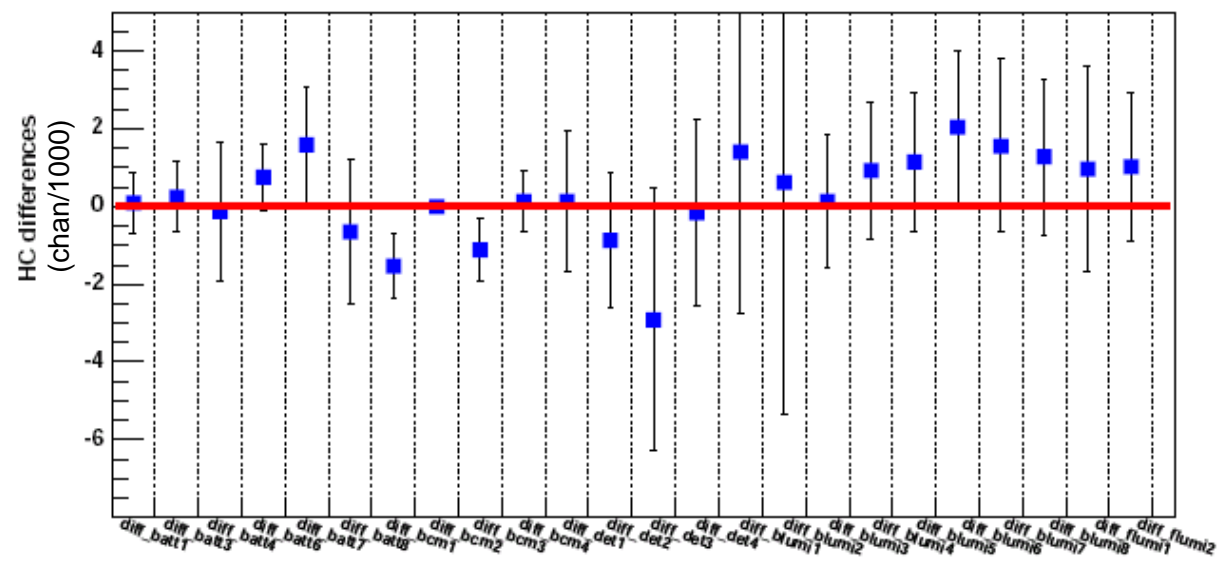

Figure 5.10. 2005 helicity-correlated crosstalk (chan/1000) for each device in the DAQ listed along the $x$ axis. The data was accumulated over 120 hours. 1 chan/1000 corresponds to $\sim 10 \mathrm{ppb}$.

the only real-time electronic helicity signal is located at the polarized source. All other helicity signals carrying helicity to the DAQ are delayed by eight helicity windows. An additional way to suppress the effects of electronic pickup is the use of the IHWP as a slow reversal of the sign of the asymmetry. The IHWP reverses the sign of all helicity information carried by the beam polarization; therefore, the physics asymmetry will change sign with the insertion of the IHWP. Because the electronic helicity signal is unchanged, any pickup of the signal will be the same sign for each IHWP state and will cancel when calculating the final asymmetry.

We measured the helicity-correlated crosstalk by measuring the asymmetry of batteries being read out by ADCs in our DAQ which should be insensitive to any helicity information, and we also measured the asymmetry of all the DAQ signals while taking pedestal data. The pedestal data is taken while the helicity Pockels cell is pulsed with $\mathrm{HV}$ and no beam is being delivered to the hall. The results of these studies for the 2005 data is presented in Figure 5.10 where the helicity-correlated differences for all signals are consistent with zero within the error bars. 


\subsubsection{Linearity}

A flux-integrating detector is designed to output a signal which is linearly proportional to the scattered flux. A nonlinearity in the PMT response to the scattered flux can be a significant systematic error. It is also true that a nonlinearity in any device which is integrated can contribute to a systematic error in the asymmetry. The following discussion concentrates only on the detectors. A nonlinearity in the detector response $D$ to the flux $F$ can be represented as:

$$
D(F)=F+\epsilon F^{2} .
$$

The flux is proportional to the product of the cross section $\sigma$ and beam current $I$. It can be shown (assuming $|\epsilon| \ll 1$ ) that the raw asymmetry is then:

$$
A_{\text {raw }}=A_{P V}+X\left(A_{P V}+A_{Q}\right)
$$

where $X=\epsilon F$ is the nonlinearity, $A_{Q}$ is the charge asymmetry and $A_{P V}$ is the physics asymmetry defined as:

$$
A_{Q}=\frac{I_{R}-I_{L}}{I_{R}+I_{L}} \text { and } A_{P V}=\frac{\sigma_{R}-\sigma_{L}}{\sigma_{R}+\sigma_{L}}
$$

The consequences of this result are that $A_{Q}$ needs to be smaller than the physics asymmetry, and the resulting fractional systematic error in the asymmetry is equal to the nonlinearity $X$.

If we consider correcting the unnormalized detector asymmetry, we have:

$$
A_{\mathrm{corr}}=A_{\mathrm{det}}-A_{Q}+\sum_{i=1}^{5} \beta_{i} \Delta x_{i}
$$

If we then also consider making a correction for the linearity, we have to consider the detector linearity as well as the BCM linearity for the $A_{Q}$ measurement and Equation 5.19 becomes:

$$
A_{\mathrm{corr}}=\left(\frac{1}{L_{Q}}\right)\left(\frac{1}{L_{D}}\right) A_{\mathrm{det}}-\left(\frac{1}{L_{Q}}\right) A_{Q}+\sum_{i=1}^{5} \beta_{i} \Delta x_{i}
$$

where $L_{Q}$ is $\left(1-X_{Q}\right)$, the BCM linearity, and $L_{D}$ is $\left(1-X_{D}\right)$, the individual detector linearity. 


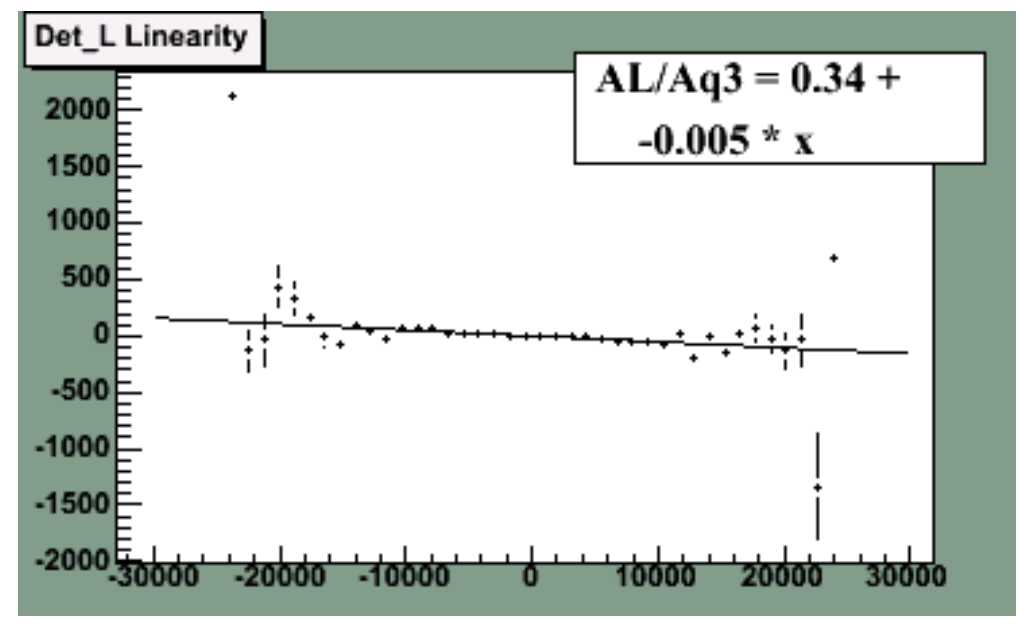

Figure 5.11. Normalized left detector asymmetry (ppm) versus BCM3 charge asymmetry (ppm). The relative nonlinearity is $-0.5 \%$.

An upper limit on the BCM nonlinearity is estimated by measuring the "double difference" between two BCMs versus the charge asymmetry measured by a third independent BCM. The double difference is defined as:

$$
\Delta \Delta=A_{Q_{\text {up }}}-A_{Q_{\text {down }}}
$$

where $A_{Q_{\mathrm{up}}}\left(A_{Q_{\mathrm{down}}}\right)$ is the charge asymmetry measured by the upstream (downstream) BCM. The upper limit for the BCM nonlinearity is $1 \%$.

The detector nonlinearity is estimated by fitting the normalized detector asymmetry versus charge asymmetry curve to a straight line (Figure 5.11). The slopes for the detector combinations were measured to be $\leq 0.5 \%$.

Although $A_{Q}$ does not directly contribute to the systematic error in $A_{P V}$, it does contribute to the systematic error of the nonlinearity correction term. $A_{Q}$ was measured to be $2.6 \mathrm{ppm}$ in 2004 and $0.4 \mathrm{ppm}$ in 2005. No correction was made for nonlinearity, but the nonlinearity values above were assigned as systematic errors.

\subsubsection{Transverse Beam Asymmetry}

The transverse beam asymmetry $A_{T}$ arises from the interference between one-photon $(1 \gamma)$ and two-photon $(2 \gamma)$ exchange when elastically scattering transversely polarized elec- 
trons from an unpolarized target. The asymmetry manifests itself due to an azimuthal modulation because

$$
A_{T} \propto \vec{S}_{e} \cdot \frac{\vec{k}_{e} \times \vec{k}_{e}^{\prime}}{\left|\vec{k}_{e} \times \vec{k}_{e}^{\prime}\right|}
$$

where $\vec{S}_{e}$ and $\vec{k}_{e}$ are the spin vector and momentum vector, respectively, of the incoming electron and $\vec{k}_{e}^{\prime}$ is the momentum vector of the scattered electron.

This asymmetry is a concern for the HAPPEX experiment because even though the beam is primarily longitudinally polarized, there is a small transverse component. The horizontal acceptance of the Hall A HRS means that HAPPEX is only sensitive to $A_{T}$ that arises from vertical beam polarization.

$A_{T}$ is oppositely signed in the left and right detectors; therefore, it contributes as a false asymmetry to the parity-violating asymmetry if it does not cancel perfectly when averaging the detectors. The cancellation is mostly affected by the symmetric alignment of the HRS and detectors in the hall. $A_{T}$ is on the order of a few ppm with a $100 \%$ polarized beam, so its contribution to the measured asymmetry should be small during production running.

$A_{T}$ was measured during the 2004 data-taking period with a total of $3.1 \times 10^{5}$ pairs with a resolution of $610 \mathrm{ppm}$ for two slugs. The same analysis techniques used for the parity-violating asymmetry are also used for the transverse analysis.

The corrected asymmetry pulse-pair distributions and the correlation of the asymmetry with IHWP state are shown in Figures 5.12 and 5.13 respectively

After correcting for helicity-correlated beam asymmetries, the results are:

$$
\left\langle A_{T}\right\rangle_{L}=5.93 \pm 1.59 \mathrm{ppm} \text { and }\left\langle A_{T}\right\rangle_{R}=-4.07 \pm 1.51 \mathrm{ppm}
$$

After correcting for polarization $(75 \%)$ and sign, the average $A_{T}$ is

$$
A_{T}^{\mathrm{H}}=-6.58 \pm 1.47 \text { (stat) } \pm 0.24 \text { (syst) ppm. }
$$

Once $A_{T}$ has been measured, the contribution to the parity-violating asymmetry can be calculated as long as the amount of vertical polarization during the production run is known. A description of how the vertical polarization was setup for the measurement of $A_{T}$ 


\section{Left HRS}

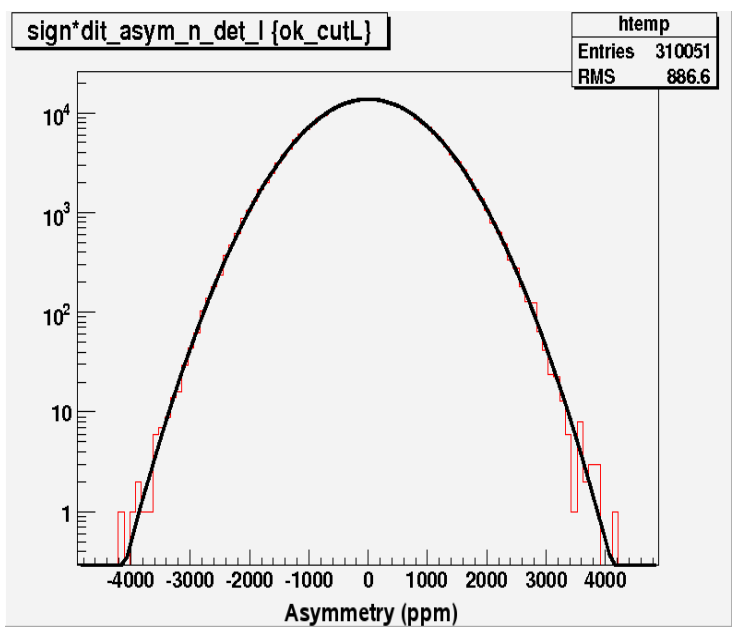

\section{Right HRS}

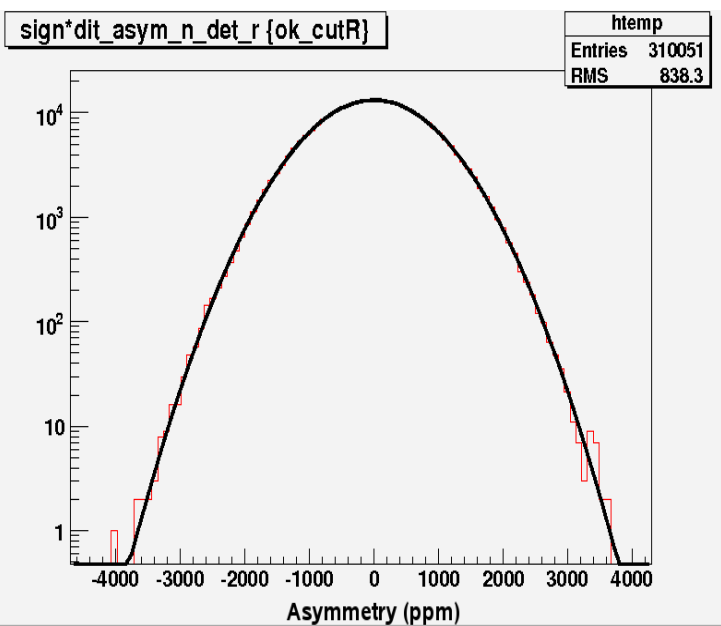

Figure 5.12. Transverse asymmetry pair plot for the left and right HRS.
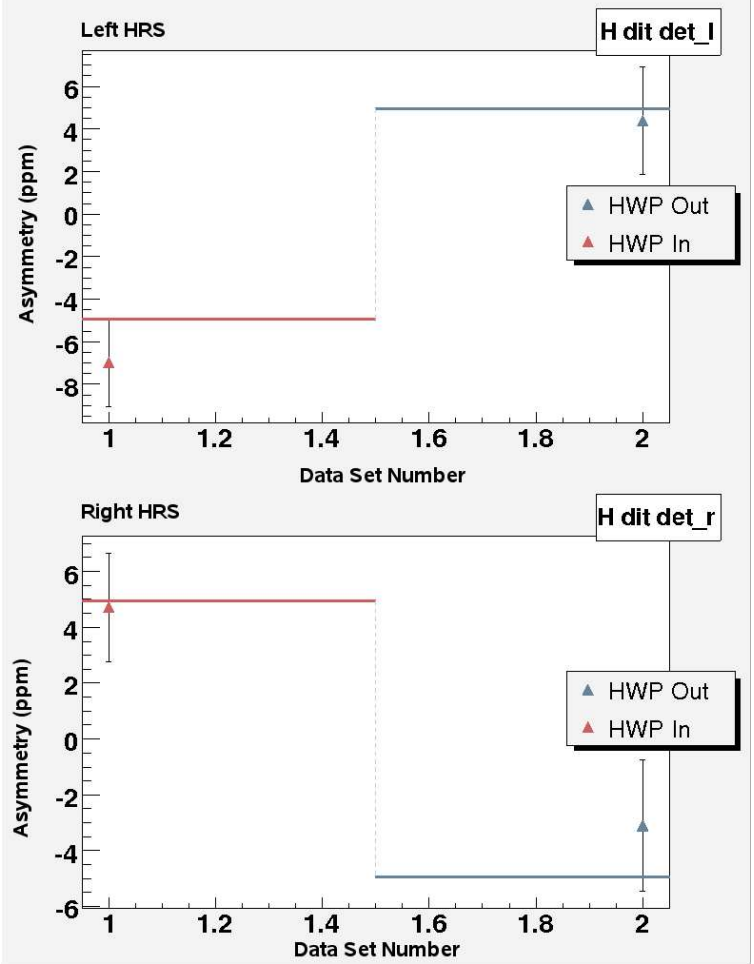

Figure 5.13. Transverse asymmetry slug plot for the left and right HRS.

can be found in [47] where the estimate of vertical polarization during HAPPEX production running is also discussed. 
From [47], the vertical polarization during the 2004 production running was $1.0 \pm 1.6 \%$. The total correction $T$ (from Equation 5.1) to $A_{\text {raw }}$ is calculated by

$$
T=\left\langle A_{T}\right\rangle_{L} P_{v} w_{L}^{\prime}+\left\langle A_{T}\right\rangle_{R} P_{v} w_{R}^{\prime}
$$

where $P_{v}$ is the vertical polarization during production running and $w_{L}^{\prime}\left(w_{R}^{\prime}\right)$ is the weight for the left (right) detector combination normalized to one. The 2004 correction was calculated to be $8 \mathrm{ppb}$. Because the size of the correction is much less than the statistical error of the measurement, it is unnecessary to make a correction to $A_{\text {raw }}$ for the transverse beam asymmetry. The $A_{T}$ systematic error is $14 \mathrm{ppb}$ for the 2004 data set. The $2005 A_{T}$ systematic error is only $4 \mathrm{ppb}$ because the vertical polarization was estimated to be $0 \pm$ $2 \%$.

\section{3 $Q^{2}$ Determination}

$Q^{2}$ is the square of the four-momentum transferred from the incident electron to the target proton and is defined by:

$$
Q^{2}=-q^{2}=-\left(q_{i}-q_{f}\right)^{2}=2 E E^{\prime}(1-\cos \theta)
$$

where $q_{i}$ and $E$ are the four-momentum and energy, respectively, of the incoming electron and $q_{f}, E^{\prime}$, and $\theta$ are the four-momentum, energy, and angle of the scattered electron. For elastic scattering, $E, E^{\prime}$, and $\theta$ are not independent which provides a consistency check for the $Q^{2}$ measurement. A detailed discussion of the $Q^{2}$ measurements for 2004 and 2005 can be found in $[48,49]$.

The beam energy was measured to be $3.03 \mathrm{GeV}$ for the 2004 run period and $3.18 \mathrm{GeV}$ for the 2005 run period. For the $Q^{2}$ calculation, a $3 \mathrm{MeV}$ average loss of energy in the target was assumed.

The central scattering angle was precisely determined using a nuclear recoil method for which we used the water cell target because it contains Hydrogen and heavy nuclei. 


\begin{tabular}{|l|c|c|}
\hline & $2004\left(\mathrm{GeV}^{2}\right)$ & $2005\left(\mathrm{GeV}^{2}\right)$ \\
\hline \hline$Q_{L}^{2}$ & $0.1018 \pm 0.001$ & $0.1107 \pm 0.0011$ \\
$Q_{R}^{2}$ & $0.0957 \pm 0.001$ & $0.1070 \pm 0.0011$ \\
$\left\langle Q^{2}\right\rangle$ & 0.0987 & 0.1089 \\
\hline
\end{tabular}

Table 5.6. ADC weighted $Q^{2}$ for left and right detectors and the average $Q^{2}$ used for the final asymmetry.

This method measures the energy of elastically scattered electrons off of different nuclei to determine the angle of the spectrometers by:

$$
E^{\prime}+\varepsilon^{\prime}=\frac{E_{0}-\varepsilon_{0}-\frac{1}{2 m}\left(m^{* 2}-m^{2}\right)}{1+(1-\cos \theta)\left(E_{0}-\varepsilon_{0}\right) / m}
$$

where $E^{\prime}$ is the measured energy of the scattered electron, $E_{0}$ is the incident beam energy, $\theta$ is the scattering angle, $m$ is the mass of the target nucleus, $m^{*}$ is the mass of the recoil nucleus, and $\varepsilon_{0}$ and $\varepsilon^{\prime}$ are the energy loss of the incident and detected electrons.

The spectrometer matrix elements were optimized for the HAPPEX optics and kinematics for reliable reconstruction of event tracks from the focal plane back to the target. This is especially important because we use an extended target $(20 \mathrm{~cm})$ for the experiment.

The $Q^{2}$ distribution is measured using a beam current of 1-2 $\mu \mathrm{A}$ in order to decrease the scattering rate to a level where the Hall A vertical drift chambers can be used for tracking events. A sample $Q^{2}$ distribution for the left and right detector combinations from 2004 is shown in Figure 5.14. The final ADC weighted $Q^{2}$ values and the average $Q^{2}$ weighted by the detectors' statistical widths are summarized in Table 5.6. The systematic error on $Q^{2}$ is $1 \%$.

The UMass $Q^{2}$ profile scanners were built to measure the $Q^{2}$ profile at production currents $\left(35\right.$ to $55 \mu \mathrm{A}$ ) to verify that the actual $Q^{2}$ distribution matches the measured distribution at low current. Regular $Q^{2}$ scans were completed to measure possible drifts of the $Q^{2}$ distribution in time. A sample $Q^{2}$ scanner distribution for the left and right detectors from 2005 is shown in Figure 5.15. 

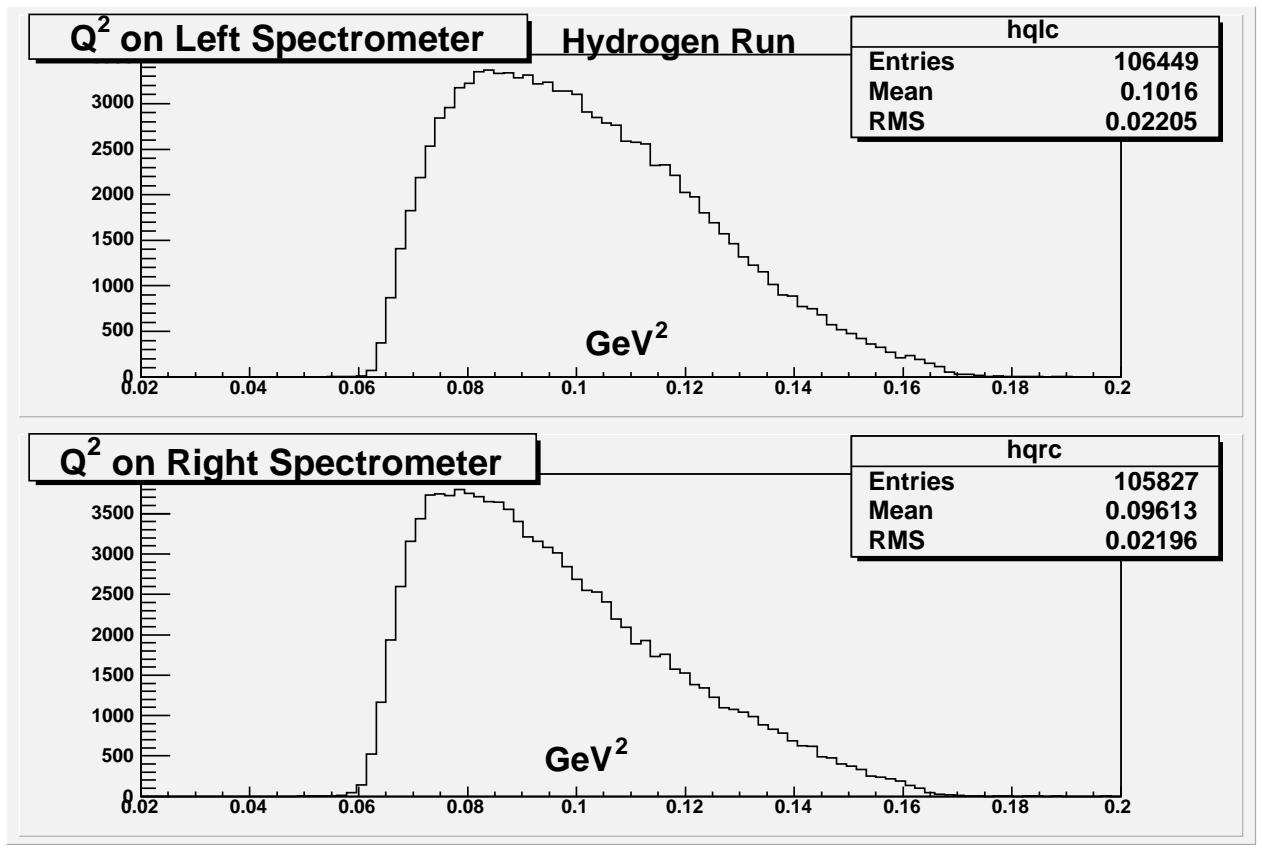

Figure 5.14. A representative sample of the left and right detector $Q^{2}$ distributions from 2004.

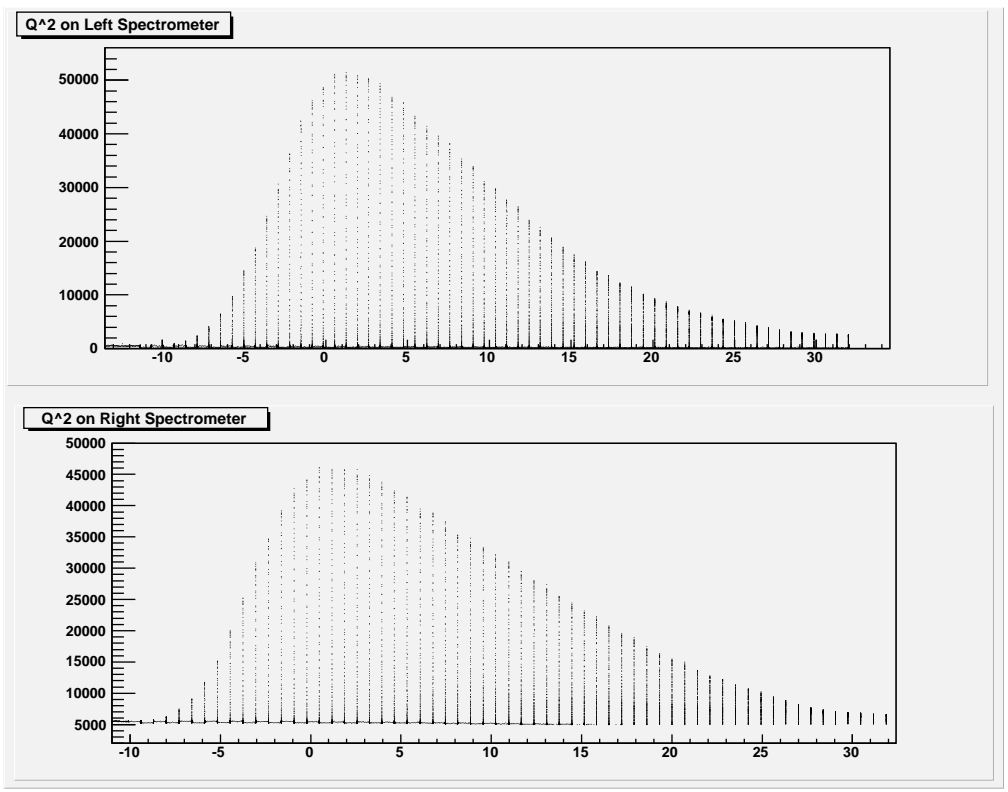

Figure 5.15. A representative sample of the left and right detector $Q^{2}$ scanner distributions from 2005. The $x$ scale is in $\mathrm{cm}$ with an arbitrary zero, and the $y$ scale is the current normalized scanner signal in ADC channels. 


\subsection{Backgrounds}

The high momentum resolution of the $\operatorname{HRS}(\delta p / p<0.1 \%)$ serves as a powerful way to focus the elastically scattered electrons into a compact region separating these events from those that are inelastically scattered in the target. This is very important for HAPPEX because the integrating technique used to measure the asymmetry does not allow for separation of background events from the desired events in the data. Dedicated runs are used to measure the background contamination in the detector flux and is discussed below. The HRS provides an extremely clean environment for the detector flux with less than $1 \%$ of the scattered flux in the detector coming from inelastic scattering.

\subsubsection{Quasi-elastic Scattering}

The dominant source of background comes from quasi-elastic scattering from the aluminum target windows. Dedicated runs using aluminum foils to mimic the production target window position and full target radiation length are used to measure the fraction of these events that contribute to the detector flux. The contribution was computed to be 0.90 $\pm 0.15 \%$ in 2004 and $0.76 \pm 0.25 \%$ in 2005 . The theoretical parity-violating asymmetry for this process was calculated as described in [50] to be $-1.7 \mathrm{ppm}(2004)$ and $-2.5 \mathrm{ppm}$ (2005). A $100 \%$ systematic error was assigned to the asymmetry to account for kinematic variations and resonance contributions. Figure 5.16 shows the particle spectrum for a low current run where the shaded region of the inset shows the foil spectrum scaled to match the contribution from the target windows.

\subsubsection{Rescattering in the HRS}

Electrons which inelastically scatter in the target cryogen can reach the detector if they rescatter in the spectrometer walls. The background fraction is proportional to the product of the rescattering probability and the ratio of the inelastic to elastic cross sections. Dedicated runs where the spectrometer central momentum was varied to study the change in the detector rates and map out the inelastic spectrum were used to calculate the background fraction. The procedure used is similar to what was done for the first-generation HAPPEX and is described in detail in [19]. The background fraction was determined to be $0 \pm 0.002$ 


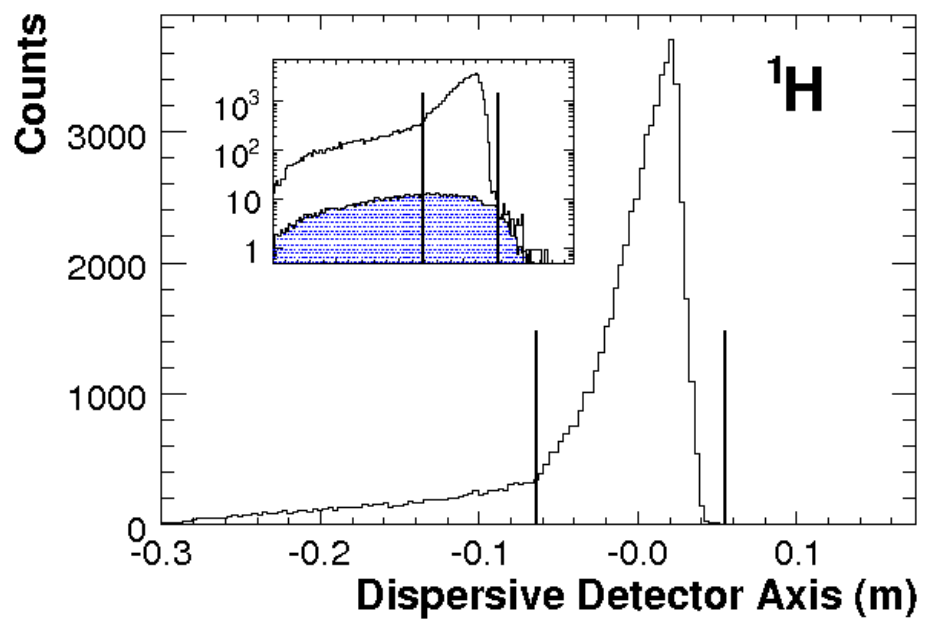

Figure 5.16. Single-particle spectrum obtained in dedicated low-current runs. The inset shows the same spectra on a logarithmic scale. The vertical lines indicate the extent of the detector. The shaded region shows the contribution from target windows.

for 2004 and $0.001 \pm 0.0005$ for 2005 where the main contributions are from the elastic radiative tail and $\Delta$ resonance scattering.

There is also concern that the scattered electrons will rescatter off of polarized iron in the HRS dipole. This is a concern because of the polarization dependent asymmetry in $e-e$ scattering. Using the information from the study performed in [19], an upper limit on the fraction was determined to be $2.4 \times 10^{-6}$ with a $100 \%$ error bar which is completely negligible.

Because the fractions for both rescattering processes are 0 for the 2004 data, no corrections were made for these backgrounds, but they do contribute to the systematic error on $A_{P V}$. The calculated asymmetries for 2004 are $-9.6 \mathrm{ppm}$ and $100 \mathrm{ppm}$ for the unpolarized and polarized rescattering processes respectively. The 2005 calculations are $-4 \mathrm{ppm}$ and $130 \mathrm{ppm}$ for the unpolarized and polarized processes. The polarized scattering asymmetry is an upper limit estimated for the systematic error. The systematic error for all the asymmetries was taken to be $100 \%$. 


\subsection{Finite Kinematic Acceptance}

The finite acceptance of the spectrometer and radiative energy losses results in an experimental asymmetry which is convoluted over a range of $Q^{2}$. In order for the asymmetry to represent a measurement at a single $Q^{2}$, these effects must be taken into account. A Monte Carlo simulation is used to calculate the parity-violating asymmetry at the effective

$Q^{2}$ of the experiment $\left(A_{P V}^{\mathrm{eff}}\right)$ which represents point scattering at this kinematics. The spectrometer acceptance and energy losses are included in the simulation such that it properly reproduces the experiment's measured $Q^{2}$ distributions and the average parity-violating asymmetry $\left(A_{\mathrm{MC}}\right)$ is calculated. A detailed description of the simulation can be found in $[19,22]$.

The acceptance correction factor $K$ is calculated as:

$$
K=\frac{A_{P V}^{\mathrm{eff}}}{A_{\mathrm{MC}}}
$$

$K$ was calculated to be $0.976 \pm 0.006$ for 2004 and $0.979 \pm 0.002$ for 2005 . This correction properly accounts for the non-linear dependence of the asymmetry with $Q^{2}$.

\subsection{Beam Polarization}

The Compton polarimeter measures the beam polarization simultaneously with production data-taking. The beam polarization normalizes the measured asymmetry and is the source of the biggest correction to the measured asymmetry.

For the 2004 HAPPEX run, the superlattice (Gun 3) and strained-layer (Gun 2) cathodes were used to provide polarized beam which provided two distinct periods of beam polarization. The 2004 results of the Compton analysis are shown in Figure 5.17. The beam polarization results after correcting for the laser polarization are:

$$
\begin{gathered}
P_{3}=89.22 \pm 0.13(\text { stat }) \pm 2.2(\text { syst }) \% \\
P_{2}=76.30 \pm 0.12(\text { stat }) \pm 1.9(\text { syst }) \% .
\end{gathered}
$$




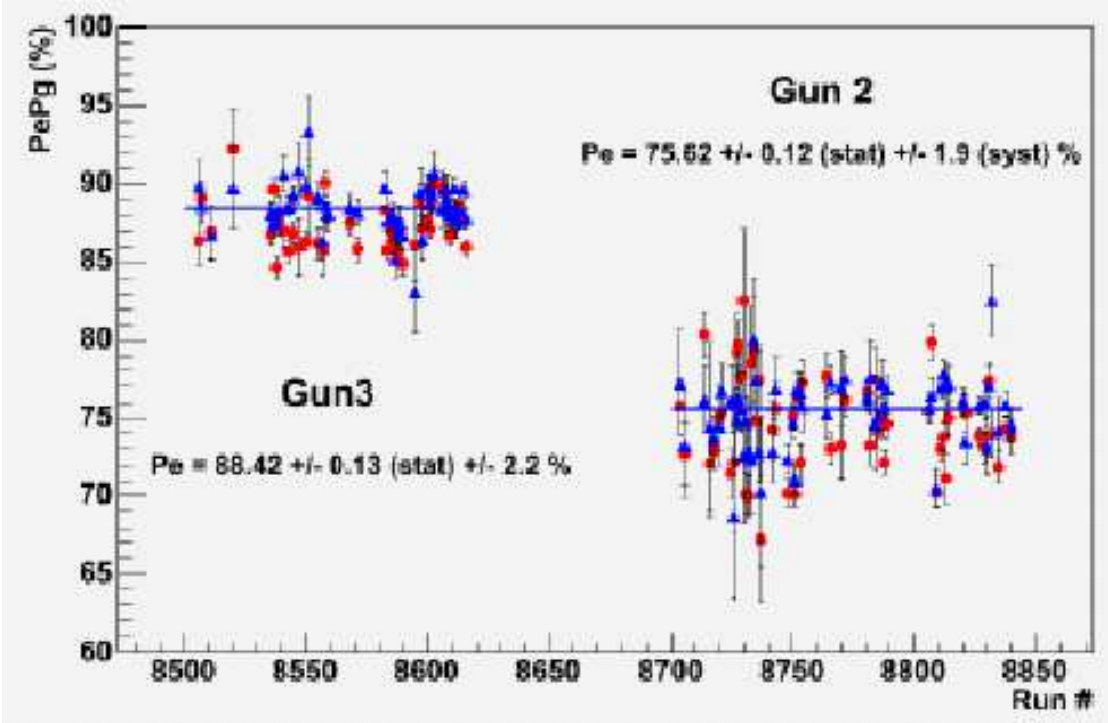

Figure 5.17. The beam polarization measured by the Compton polarimeter for the 2004 data run. Gun 3 and Gun 2 refer to the periods of time when the superlattice and strainedlayer cathodes, respectively, were in use. The polarization numbers are $P_{e} P_{\gamma}$.

The average polarization was calculated by weighting each cathode period by the fraction of data accumulated in each period. The weights used in calculating the average are 0.387 for Gun 3 and 0.613 for Gun 2 for an average polarization:

$$
P_{b}=81.30 \pm 1.6 \text { (syst) } \% \text {. }
$$

The 2005 beam polarization was measured to be $P_{b}=87.1 \pm 0.81$ (syst)\%.

\subsection{Physics Asymmetry}

The final physics asymmetry is calculated by:

$$
A_{P V}=\frac{K}{P_{b}} \frac{\left\langle A_{\text {corr }}\right\rangle-P_{b} \sum_{i} A_{i} f_{i}}{1-\sum_{i} f_{i}}
$$

using the values for each term and factor as discussed throughout this chapter and summarized in Table 5.7. The corrections to the asymmetry are summarized in Table 5.8. 


\begin{tabular}{|l|c|c|c|c|c|}
\hline \multicolumn{2}{|c|}{} & \multicolumn{2}{|c|}{2004} & \multicolumn{2}{c|}{2005} \\
\hline \hline Term & Description & Value & Syst. Error & Value & Syst. Error \\
\hline$F$ & False asyms & $-79 \mathrm{ppb}$ & $35 \mathrm{ppb}$ & $-10 \mathrm{ppb}$ & $15 \mathrm{ppb}$ \\
$P_{b}$ & Polarization & 0.813 & 0.016 & 0.871 & 0.0081 \\
$L_{Q}$ & BCM nonlinearity & 1.0 & 0.01 & 1.0 & 0.02 \\
$L_{D}$ & Det nonlinearity & 1.0 & 0.002 & 1.0 & 0.01 \\
$Q$ & $Q^{2}$ correction & 1.0 & 0.01 & 1.0 & 0.01 \\
$K$ & Acceptance corr & 0.976 & 0.006 & 0.979 & 0.002 \\
$f_{1}$ & Al QE fraction & 0.0091 & 0.0015 & 0.0076 & 0.0025 \\
$A_{1}$ & Al QE asym & $-1.7 \mathrm{ppm}$ & $1.7 \mathrm{ppm}$ & $-2.5 \mathrm{ppm}$ & $2.5 \mathrm{ppm}$ \\
$f_{2}$ & Rescatter frac & 0 & 0.002 & 0.001 & 0.0005 \\
$A_{2}$ & Rescatter asym & $-9.56 \mathrm{ppm}$ & 9.56 & $-4 \mathrm{ppm}$ & $4 \mathrm{ppm}$ \\
$f_{3}$ & Pole-tip frac & 0 & $2.5 \times 10^{-4}$ & $2.5 \times 10^{-6}$ & $2.5 \times 10^{-6}$ \\
$A_{3}$ & Pole-tip asym & $100 \mathrm{ppm}$ & 100 & $130 \mathrm{ppm}$ & 130 \\
\hline
\end{tabular}

Table 5.7. Summary of all the $A_{P V}$ terms and factors with the associated systematic errors on each.

\begin{tabular}{|l|c|c|}
\hline Correction $(\mathrm{ppb})$ & 2004 & 2005 \\
\hline \hline Beam Asyms. & $-79 \pm 32$ & $-10 \pm 17$ \\
Target Windows & $6 \pm 16$ & $7 \pm 19$ \\
Rescatter & $0 \pm 31$ & $2 \pm 4$ \\
Nonlinearity & $0 \pm 15$ & $0 \pm 15$ \\
\hline \hline Normalization Factor & 2004 & 2005 \\
\hline \hline Acceptance $K$ & $0.976 \pm 0.006$ & $0.979 \pm 0.002$ \\
$Q^{2}$ Scale & $1.000 \pm 0.015$ & $1.000 \pm 0.017$ \\
Polarization $P_{b}$ & $0.813 \pm 0.016$ & $0.871 \pm 0.009$ \\
\hline
\end{tabular}

Table 5.8. Corrections to $A_{\text {raw }}$ and systematic errors for HAPPEX 2004 and 2005. 


\begin{tabular}{|l|c|c|c|c|}
\hline Description & \multicolumn{2}{|c|}{$\begin{array}{c}\text { Eor Contributed } \\
\text { Error (ppb) }\end{array}$} & $\begin{array}{c}\text { 2005 Contributed } \\
\text { Error (ppb) }\end{array}$ \\
\hline \hline False Asymmetry & 36 & 43 & 14 & 17 \\
$\quad$ Position/Angle & 14 & & 0 & \\
Energy & 17 & & 4 & \\
$A_{T}$ & & 23 & & 15 \\
Polarization & & 15 & & 15 \\
Linearity & 11 & & 9 & \\
BCM & 10 & & 11 & \\
Det & & 7 & & 3 \\
Acceptance & & 12 & & 27 \\
Q & & 34 & & 20 \\
Backgrounds & 16 & & 19 & \\
$\quad$ Al QE & 17 & & 4 & \\
Rescatter & 26 & & 3 & \\
Pole-tip & & 63 & & 43 \\
\hline \hline Total Systematic Error & & &
\end{tabular}

Table 5.9. Summary of all contributions to the $A_{P V}$ systematic error.

After all corrections, the 2004 result at $Q^{2}=0.099 \mathrm{GeV}^{2}$ is

$$
A_{P V}=-1.14 \pm 0.24 \text { (stat) } \pm 0.06 \text { (syst) ppm. }
$$

After all corrections, the 2005 result at $Q^{2}=0.109 \mathrm{GeV}^{2}$ is

$$
A_{P V}=-1.58 \pm 0.12 \text { (stat) } \pm 0.04 \text { (syst) ppm. }
$$

The contribution of the errors in the corrections to the final systematic error of $A_{P V}$ is obtained by following the normal error propagation procedure for a function of uncorrelated quantities $f\left(u_{i}\right)$ :

$$
\sigma_{f}^{2}=\sum_{i} \sigma_{u_{i}}^{2}\left(\frac{\partial f}{\partial u_{i}}\right)^{2}
$$

where $f$ is $A_{P V}$ in this case. The contributed systematic errors for the corrections made to $A_{P V}$ are summarized in Table 5.9. 


\section{CHAPTER 6}

\section{RESULTS AND CONCLUSIONS}

In this chapter, I will discuss the extraction of the strange vector form factors from the measured parity-violating asymmetry, interpretation of the results, and future plans for parity-violation experiments at Jefferson Lab.

\subsection{Results}

The tree-level parity-violating asymmetry for elastic electron-proton scattering introduced in Chapter 1 must be modified to consider electroweak radiative corrections for comparison to the measured asymmetry. The asymmetry is given in the standard model (and with the assumption of charge asymmetry) by [18]:

$$
\begin{aligned}
A_{P V}= & -\frac{G_{F} Q^{2}}{4 \pi \alpha \sqrt{2}} \times\left\{\left(1+R_{V}^{p}\right)\left(1-4 \sin ^{2} \theta_{W}\right)\right. \\
& -\left(1+R_{V}^{n}\right) \frac{\epsilon G_{E}^{\gamma p} G_{E}^{\gamma n}+\tau G_{M}^{\gamma p} G_{M}^{\gamma n}}{\epsilon\left(G_{E}^{\gamma p}\right)^{2}+\tau\left(G_{M}^{\gamma p}\right)^{2}}-\left(1-R_{V}^{(0)}\right) \frac{\epsilon G_{E}^{\gamma p} G_{E}^{s}+\tau G_{M}^{\gamma p} G_{M}^{s}}{\epsilon\left(G_{E}^{\gamma p}\right)^{2}+\tau\left(G_{M}^{\gamma p}\right)^{2}} \\
& \left.-\frac{\left(1-4 \sin ^{2} \theta_{W}\right) \epsilon^{\prime} G_{M}^{\gamma p}}{\epsilon\left(G_{E}^{\gamma p}\right)^{2}+\tau\left(G_{M}^{\gamma p}\right)^{2}}\left[-2\left(1+R_{A}^{T=1}\right) G_{A}^{T=1}+\left(\sqrt{3} R_{A}^{T=0}\right) G_{A}^{T=0}\right]\right\}
\end{aligned}
$$

with

$$
\tau=\frac{Q^{2}}{4 M_{p}^{2}}, \quad \epsilon=\left[1+2(1+\tau) \tan ^{2}\left(\frac{\theta}{2}\right)\right]^{-1}, \quad \text { and } \quad \epsilon^{\prime}=\sqrt{\tau(1+\tau)\left(1-\epsilon^{2}\right)}
$$

where $G_{F}$ is the Fermi constant, $\alpha$ is the fine structure constant, and $\theta_{W}$ is the electroweak mixing angle. $G_{E(M)}^{\gamma p}$ is the proton electric (magnetic) form factor, $G_{E(M)}^{\gamma n}$ is the neutron

electric (magnetic) form factor, and $G_{A}^{T=1(0)}$ is the isovector (isoscalar) proton axial form factor, all of which are functions of $Q^{2}$. The $R_{V(A)}$ factors are radiative corrections of 


\begin{tabular}{|c|c|}
\hline \multicolumn{2}{|c|}{ Electroweak Radiative Corrections } \\
\hline \hline$R_{V}^{p}$ & -0.045 \\
$R_{V}^{n}$ & -0.0118 \\
$R_{V}^{(0)}$ & 0.0117 \\
$R_{A}^{T=1}$ & $-0.173+[-0.086 \pm 0.346$ (anapole) $]$ \\
$R_{A}^{T=0}$ & $-0.253+[-0.014 \pm 0.202$ (anapole) $]$ \\
\hline
\end{tabular}

Table 6.1. Vector and axial electroweak radiative correction factors (using the $\overline{\mathrm{MS}}$ renormalization scheme) $[51,52]$ for the calculation of $A_{P V}^{(s=0)}$.

\begin{tabular}{|c|c|c|}
\hline $\begin{array}{c}\text { Effective } \\
\text { Kinematics }\end{array}$ & 2004 & 2005 \\
\hline $\begin{array}{l}E_{\text {eff }} \\
Q_{\text {eff }}^{2} \\
\theta_{\text {eff }} \\
\end{array}$ & $\begin{array}{c}3.0228 \mathrm{GeV} \\
0.0986 \mathrm{GeV}^{2} \\
6.007^{\circ}\end{array}$ & $\begin{array}{c}3.176 \mathrm{GeV} \\
0.1089 \mathrm{GeV}^{2} \\
6.0112^{\circ}\end{array}$ \\
\hline \multicolumn{3}{|c|}{ Dimensionless Kinematic Factors } \\
\hline $\begin{array}{l}\tau \\
\epsilon \\
\epsilon^{\prime}\end{array}$ & $\begin{array}{c}0.02800 \\
0.9944 \\
0.01798\end{array}$ & $\begin{array}{c}0.03093 \\
0.9943 \\
0.01896\end{array}$ \\
\hline
\end{tabular}

Table 6.2. Values of the effective kinematics and dimensionless kinematic factors used for the $A_{P V}^{(s=0)}$ calculation.

the neutral weak current and are independent of $Q^{2}$. The numerical values for $R_{V(A)}$ are summarized in Table 6.1.

The vector and axial corrections are calculated using the minimum subtraction $(\overline{\mathrm{MS}})$ renormalization scheme where $\sin ^{2} \theta_{W} \equiv \sin ^{2} \hat{\theta}\left(M_{Z}\right)=0.2312$ [51]. The $R_{A}^{T=0(1)}$ factors include the electroweak corrections given by [51], and additionally the anapole contribution (a parity-violating coupling of the photon to the proton) to the radiative corrections has been calculated by [52].

Purely electromagnetic radiative corrections to the parity-violating asymmetry are negligible. The momentum acceptance of the detector, $\delta p / p<3 \%$, is such that only soft photons (photons of maximum energy $\Delta E=0.03 E \ll E, E^{\prime}$ ) reach the detector. In the soft photon limit, the electromagnetic corrections of the elastic cross section are independent of spin such that they can be expressed as a multiplicative factor to the cross section [53]; and therefore the corrections cancel in the asymmetry. 


\begin{tabular}{|c|c|c|c|c|}
\hline Form & \multicolumn{2}{|c|}{2004} & \multicolumn{2}{c|}{2005} \\
Factor & Value & Error & Value & Error \\
\hline \hline$G_{E}^{p}$ & 0.754 & 0.019 & 0.734 & 0.007 \\
$G_{M}^{p}$ & 2.144 & 0.032 & 2.086 & 0.031 \\
$G_{E}^{n}$ & 0.035 & 0.011 & 0.037 & 0.004 \\
$G_{M}^{n}$ & -1.447 & 0.022 & -1.403 & 0.021 \\
$G_{A}^{T=1}$ & 0.526 & 0.005 & 0.517 & 0.020 \\
$G_{A}^{T=0}$ & 0.140 & 0.003 & 0.137 & 0.040 \\
\hline
\end{tabular}

Table 6.3. Values of the form factors evaluated at $Q_{\text {eff }}^{2}$ used for the $A_{P V}^{(s=0)}$ calculation.

For comparison to the measured asymmetry, the theoretical value for the parity-violating asymmetry in Equation 6.1 is calculated assuming no strange quarks $\left(G^{s}=0\right)$ contribute to $A_{P V}$ and is evaluated at the effective kinematics of the experiment. The kinematic factors are summarized in Table 6.2 for the 2004 and 2005 data-taking periods. The values of the electromagnetic form factors used for the $A_{P V}$ calculation are taken from a phenomenological fit to the world data at low $Q^{2}[16]$. The values of the form factors evaluated at the HAPPEX $Q^{2}$ and their uncertainties are listed in Table 6.3. In 2004, there was no experimental value of $G_{E}^{n}$ at low $Q^{2}$; therefore a large uncertainty in the value $G_{E}^{n}$ was assigned and contributes significantly to the uncertainty in the theoretical asymmetry. In 2005 , this error was significantly reduced with a measurement of $G_{E}^{n}$ at low $Q^{2}$ from the BLAST experiment [54].

The axial form factors and the associated radiative corrections are listed in Tables 6.3 and 6.1. The axial form factor contributions are calculated assuming a dipole form $[55,56]$. The total contribution of the axial terms to the 2004 asymmetry is $-0.026 \pm 0.008 \mathrm{ppm}$ for which no correction nor uncertainty was included for the anapole moment contribution. The total contribution of the axial terms to the 2005 asymmetry include all radiative corrections (including the anapole moment [52]) and is $-0.037 \pm 0.018 \mathrm{ppm}$. The error in the contribution of the axial form factor is dominated by the uncertainties in the anapole moment contributions. Sensitivity to possible strange quark contributions to the axial form factor are neglected. 


\begin{tabular}{|c|c|c|}
\hline & $\begin{array}{c}2004 \\
Q^{2}=0.099 \mathrm{GeV}^{2}\end{array}$ & $\begin{array}{c}2005 \\
Q^{2}=0.109 \mathrm{GeV}^{2}\end{array}$ \\
\hline \hline$A_{P V}(\mathrm{ppm})$ & $-1.14 \pm 0.24 \pm 0.06$ & $-1.58 \pm 0.12 \pm 0.04$ \\
\hline$A_{P V}^{(s=0)}(\mathrm{ppm})$ & $-1.43 \pm 0.11$ & $-1.66 \pm 0.05$ \\
\hline
\end{tabular}

Table 6.4. Summary of the measured $A_{P V}$ and the calculated $A_{P V}^{(s=0)}$ for 2004 and 2005 data sets.

Assuming no strange quarks in the nucleon $\left(G^{s}=0\right)$, the standard model predicts $A_{P V}^{(s=0)}=-1.43 \pm 0.11(\mathrm{FF}) \mathrm{ppm}$ for the 2004 kinematics $\left(Q^{2}=0.099 \mathrm{GeV}^{2}\right)$ and $A_{P V}^{(s=0)}=$ $-1.66 \pm 0.05(\mathrm{FF}) \mathrm{ppm}$ for the 2005 kinematics $\left(Q^{2}=0.109 \mathrm{GeV}^{2}\right)$. The error on this asymmetry is mainly due to the uncertainty in $G_{E}^{n}$.

Comparing the non-strange parity-violating asymmetry $\left(A_{P V}^{(s=0)}\right)$ to our measurement of $A_{P V}$ (see Table 6.4), we extract a value for the linear combination of strange form factors of

$$
G_{E}^{s}+0.080 G_{M}^{s}=0.030 \pm 0.025 \text { (stat) } \pm 0.006 \text { (syst) } \pm 0.012(\mathrm{FF})
$$

at $Q^{2}=0.099 \mathrm{GeV}^{2}$ for the 2004 data set [45]. For the 2005 data set, we extract a linear combination of the strange form factors of

$$
G_{E}^{s}+0.088 G_{M}^{s}=0.007 \pm 0.011 \text { (stat) } \pm 0.004 \text { (syst) } \pm 0.005(\mathrm{FF})
$$

at $Q^{2}=0.109 \mathrm{GeV}^{2}[46]$.

Combining these results with the HAPPEX- ${ }^{4} \mathrm{He}$ measurement which is only sensitive to $G_{E}^{s}$, we are able to separate the electric and magnetic strange form factors. The 2004 HAPPEX- ${ }^{4} \mathrm{He}$ measurement provides a value of the electric form factor, $G_{E}^{s}=-0.038 \pm$ $0.042 \pm 0.010$ at $Q^{2}=0.091 \mathrm{GeV}^{2}$ [57]. The two HAPPEX results along with the three other $A_{P V}$ measurements at $Q^{2} \sim 0.1 \mathrm{GeV}^{2}[58,59,60]$ are shown in Figure 6.1 with the $1 \sigma$ error bands (a quadrature sum of the statistical and systematic errors).

The result from SAMPLE [58] is a measurement of $G_{M}^{s}$ at $Q^{2}=0.1 \mathrm{GeV}^{2}$ obtained from a measurement of $A_{P V}$ for electron-proton scattering at backward angles which is primarily sensitive to a linear combination of $G_{M}^{s}$ and $G_{A}^{s}$. In order to isolate $G_{M}^{s}$, SAMPLE 


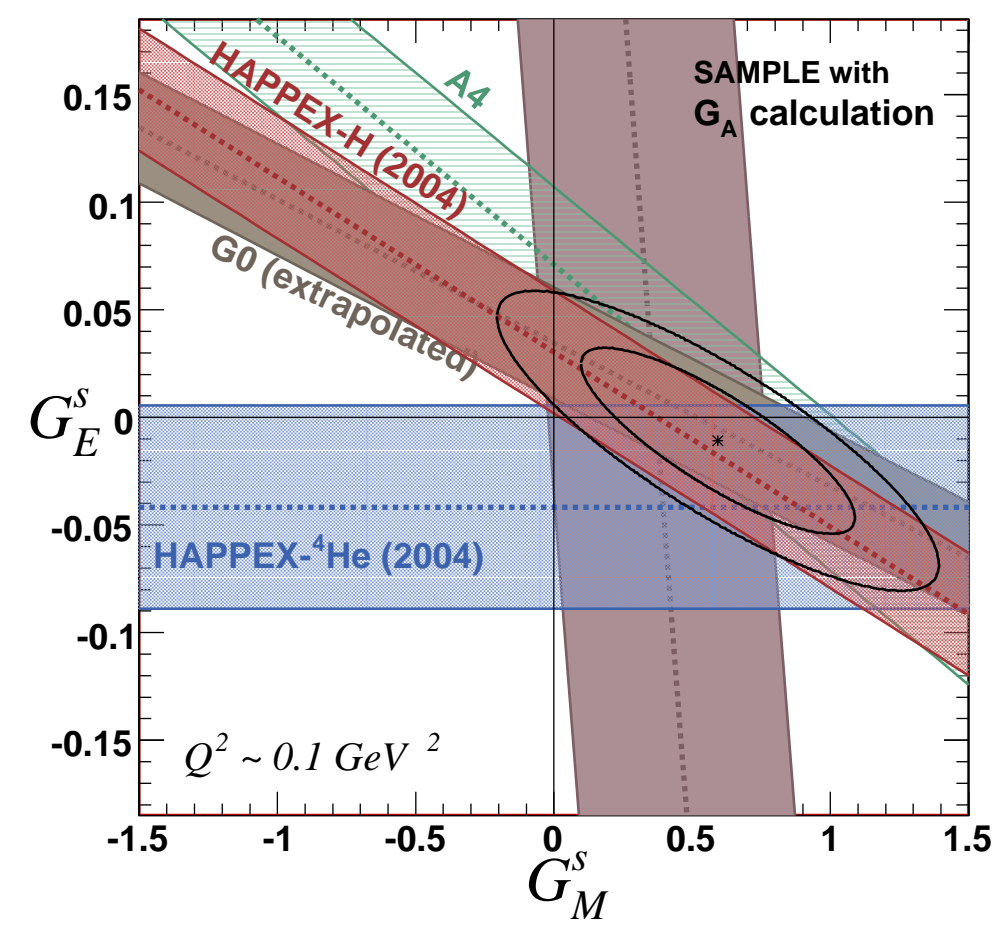

Figure 6.1. The five $A_{P V}$ measurements at $Q^{2}=0.09-0.11 \mathrm{GeV}^{2}$ are shown with shaded bands representing the 1- $\sigma$ combined statistical and systematic uncertainties. Also shown are the combined $68 \%$ and $95 \%$ C.L. ellipses from all five measurements. Only the 2004 HAPPEX results are shown here.

also measures $G_{A}^{s}$ only, by measuring the asymmetry of electron-deuterium scattering at backward angles. The SAMPLE measurements combined with the calculation of $G_{A}^{s}$ by Zhu et al. [52] is used to obtain their final results.

The A4 result [59] is a measurement of $A_{P V}$ for electron-proton scattering at forward angles $\left(30^{\circ}<\theta<40^{\circ}\right)$ and $Q^{2}=0.108 \mathrm{GeV}^{2}$. The $\mathrm{A} 4$ measurement is sensitive to the linear combination of the strange vector form factors of $G_{E}^{s}+0.106 G_{M}^{s}$.

Finally, the G0 collaboration measured $A_{P V}$ for electron-proton scattering at forward angles over a range of $Q^{2}$ values of which their lowest $Q^{2}$ bin is $0.122 \mathrm{GeV}^{2}$ [60]. Their sensitivity to the strange vector form factors is $G_{E}^{s}+0.12 G_{M}^{s}$ at this $Q^{2}$ value.

As discussed above, all measurements of the electric and magnetic strange form factors were completed at similar $Q^{2}$ values such that combining the results does not introduce any significant uncertainties. The dot in Figure 6.1 shows the best fit values of 


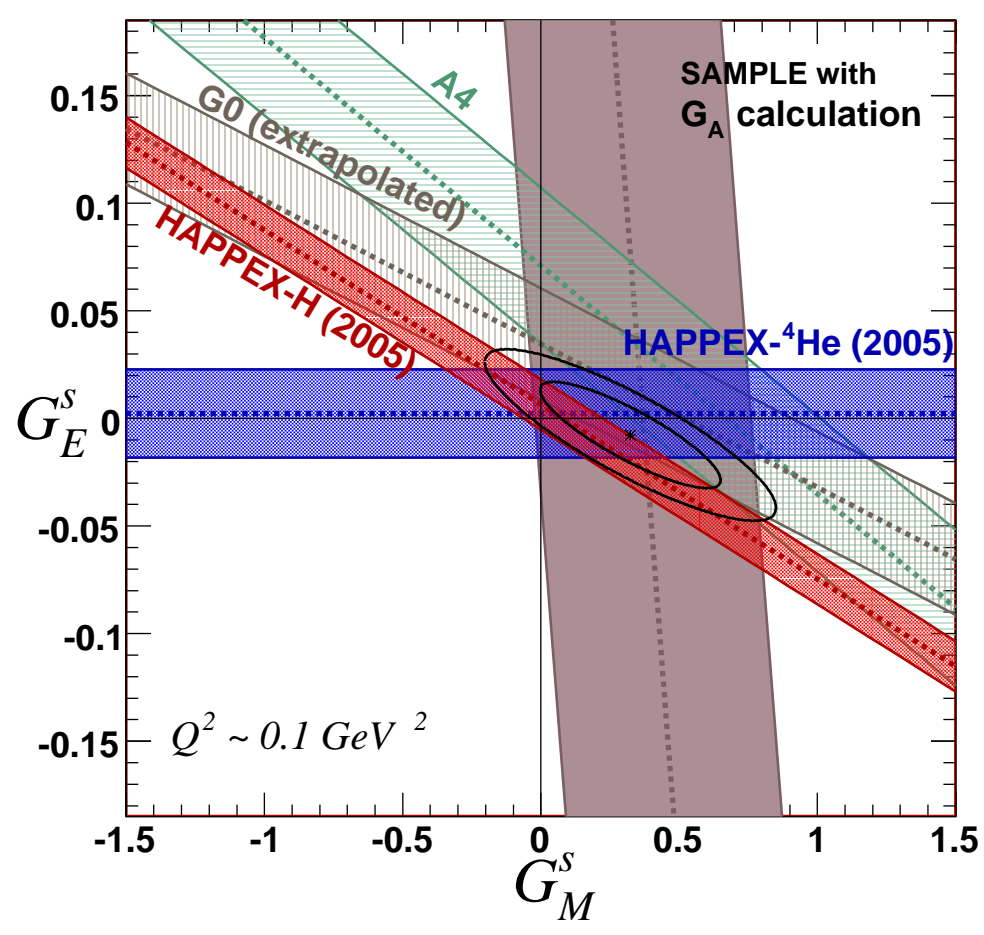

Figure 6.2. The five $A_{P V}$ measurements at $Q^{2}=0.077-0.109 \mathrm{GeV}^{2}$ are shown with shaded bands representing the 1- $\sigma$ combined statistical and systematic uncertainties. Also shown are the combined $68 \%$ and $95 \%$ C.L. ellipses from all five measurements (2004 and 2005 HAPPEX results included in the fit).

$G_{E}^{s}=-0.011 \pm 0.028$ and $G_{M}^{s}=0.594 \pm 0.327$ (with a correlation of -0.83) obtained for the five measurements. The ellipses in the figure are the 68 and $95 \%$ confidence level intervals for the combined results.

The 2005 HAPPEX results increased the precision of the HAPPEX measurement of the strange form factors by more than a factor of two. The HAPPEX-H results are given in Equation 6.3, and the HAPPEX- ${ }^{4} \mathrm{He}$ result is $G_{E}^{s}=0.002 \pm 0.014 \pm 0.007$ at $Q^{2}=0.077 \mathrm{GeV}^{2}$. These results are plotted with the other three $A_{P V}$ results discussed above and are shown in Figure 6.2. The fit in the figure includes both HAPPEX measurements and best-fit values of $G_{E}^{s}=-0.008 \pm 0.016$ and $G_{M}^{s}=0.324 \pm 0.215$ (with a correlation of -0.85) are obtained from all measurements of the strange vector form factors at $Q^{2} \sim 0.1 \mathrm{GeV}^{2}$. 
The fit of the data is performed by extrapolating all measurements to a common $Q^{2}=0.1 \mathrm{GeV}^{2}$. This extrapolation is done by assuming that $G_{E}^{s} \propto Q^{2}$ and $G_{M}^{s}$ is constant which is consistent with the assumptions made in [61]. The assumed $Q^{2}$ dependence is actually of little importance since a fit assuming both $G_{E}^{s}$ and $G_{M}^{s}$ constant gives similar results. In addition, a more elaborate $Q^{2}$ evolution, e.g. a Galster parameterization for $G_{E}^{s}$ and a dipole form for $G_{M}^{s}$ with the nucleon form factor "dipole mass," does not qualitatively change the fit results at low $Q^{2}[61,62]$.

\subsection{Interpretation and Conclusion}

The world data on strange vector form factors including only the 2004 HAPPEX measurements (Figure 6.1) was suggestive of a 5 to $10 \%$ contribution from strange quarks to the proton magnetic moment. The most recent results (Figure 6.2) are consistent with zero and suggest at most a $3.9 \pm 2.6 \%$ strangeness contribution to the proton magnetic moment and only $0.3 \pm 0.7 \%$ to the proton charge distribution.

The results from the combined 2004 and 2005 HAPPEX measurements alone are shown in Figure 6.3. From these measurements, we extract a best-fit value of $G_{E}^{s}=-0.005 \pm 0.019$ and $G_{M}^{s}=0.18 \pm 0.27$ (with a correlation of -0.87) at $Q^{2}=0.1 \mathrm{GeV}^{2}$. The HAPPEX measurements alone give consistent and similarly precise results as the world data set for the strange vector form factors at $Q^{2}=0.1 \mathrm{GeV}^{2}$. The fact that the ellipses in Figures 6.2 and 6.3 have nearly the same area is evidence that the HAPPEX results are not altered by the addition of the measurements from $[58,59,60]$. The HAPPEX results provide for a clean interpretation of the strange vector form factors because they are quite insensitive to variations in $G_{A}^{Z}$ caused by uncertainties in its determination.

Selected theoretical models predicting values for $G_{E}^{s}$ and $G_{M}^{s}$ at low $Q^{2}[63,64,65,66$, 67, 68, 69] are shown in Figure 6.3 along with the HAPPEX results. The predictions for small strange vector form factors $[67,68,69]$ are favored by the experimental results.

The measurements of the unpolarized parton distribution functions indicate that there is a sizeable net $s \bar{s}$ content which contributes significantly to the proton momentum, and several theoretical calculations $[63,64,65]$ predict large strange quark contributions to the 


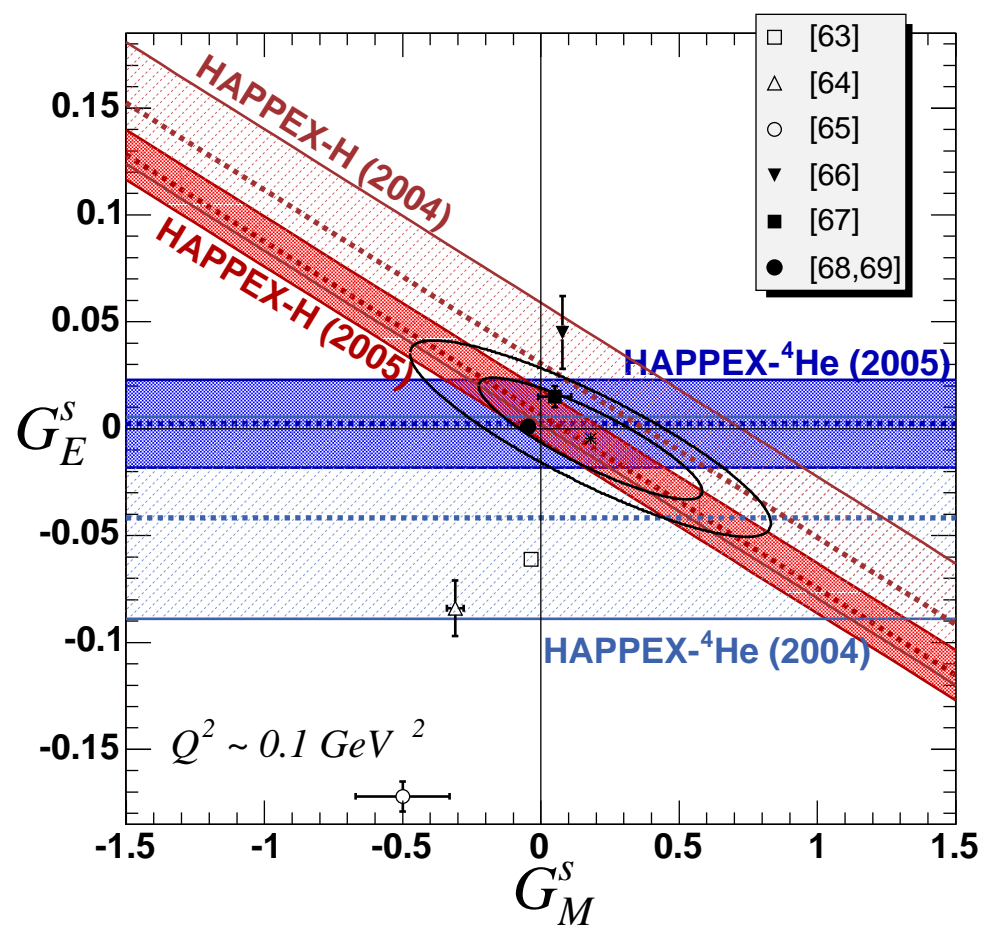

Figure 6.3. HAPPEX results with various theoretical predictions for $G_{E}^{s}$ and $G_{M}^{s}$ plotted with published uncertainty estimates, when available. The ellipses are the $68 \%$ and $95 \%$ C.L. ellipses for the combined 2004 and 2005 HAPPEX measurements.

electric and magnetic proton properties. But the present experimental results are consistent with no observable strangeness dynamics, i.e. no $s \bar{s}$ separation, within the nucleon.

The precision of the current measurement and the theoretical uncertainties, especially in the assumption of charge symmetry [70], limit any meaningful improvement of the measurement of the strange vector form factors at low $Q^{2}$.

It now becomes a challenge for the theoretical approaches to reconcile the experimental results and explain the role of strange quarks in the proton.

\subsection{Future Measurements}

Parity-violating electron scattering provides a very useful tool for understanding nucleon structure. The future measurements at Jefferson Lab aim to measure parity-violating asymmetries an order of magnitude smaller than the current measurement and with increased precision. These measurements aim to study more about strangeness in the nucleon, nuclear 


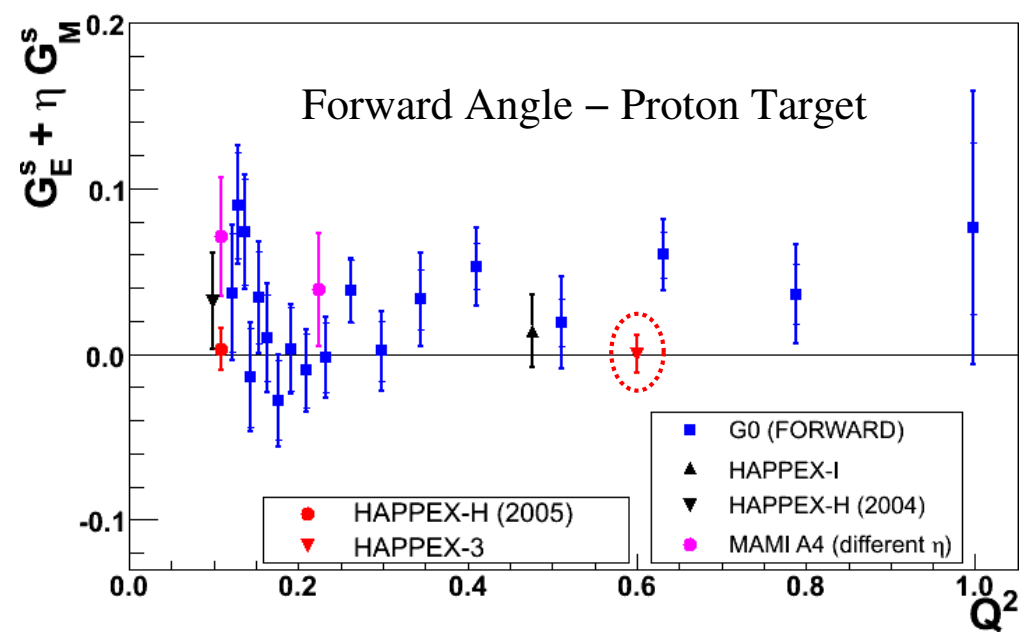

Figure 6.4. $Q^{2}$ evolution of $G_{E}^{s}+\eta G_{M}^{s}$ for current forward angle measurements [19, 45, $46,59,60]$. The expected precision for the future HAPPEX measurement at $Q^{2}=0.6 \mathrm{GeV}^{2}$ [72] is also shown.

structure, and physics beyond the Standard Model. It will be a significant experimental and theoretical challenge to control the systematics at the level necessary for accurate measurements and interpretation of the results.

\subsubsection{Strange Vector Form Factors}

Although there is no evidence for strange quark contributions to the electric and magnetic properties of the proton at low $Q^{2}$, the data from [60] suggest possible non-zero contributions at $Q^{2}>0.5 \mathrm{GeV}^{2}$. Figure 6.4 shows the linear combination $G_{E}^{s}+\eta G_{M}^{s}$ for $0<Q^{2}<1 \mathrm{GeV}^{2}$ where $\eta$ is $\tau G_{M}^{\gamma p} / \epsilon G_{E}^{\gamma p}$. A future HAPPEX measurement has been approved to measure $G_{E}^{s}+\eta G_{M}^{s}$ with high precision at $Q^{2}=0.6 \mathrm{GeV}^{2}$ in order to definitively determine if the strange quarks contribute significantly to the proton vector form factors at high $Q^{2}$. A G0 measurement at backward angle of $G_{M}^{s}$ at $Q^{2}=0.6 \mathrm{GeV}^{2}$ [71] is currently taking data. Together these two measurements will allow the determination of the strange quark contributions to the electric and magnetic structure of the proton at high $Q^{2}$.

\subsubsection{Nuclear Structure}

The PREX experiment will use parity-violating elastic electron scattering from a ${ }^{208} \mathrm{~Pb}$ nucleus in order to measure the neutron skin radius, $R_{n}$, with a precision of $1 \%$ [73]. A 
precise measurement of $R_{n}$ is a crucial parameter necessary for calculating the structure of neutron stars. In addition, $R_{n}$ is an important parameter for understanding possible exotic phases of dense matter, determining the proton fraction in neutron-rich matter, and interpreting atomic physics measurements of the electroweak mixing angle.

\subsubsection{Physics Beyond the Standard Model}

The $Q_{\text {weak }}$ experiment will measure the proton's weak charge, $1-4 \sin ^{2} \theta_{W}$, at $Q^{2}$ $=0.03 \mathrm{GeV}^{2}$ with $4 \%$ precision by measuring the parity-violating asymmetry for elastic electron-proton scattering at very-forward angle [74]. This measurement will provide a $0.3 \%$ measurement of the electroweak mixing angle improving the current precision of $\sin ^{2} \theta_{W}$ at low $Q^{2}$ by a factor of two. The measurement is sensitive to a linear combination of the $Z$-electron axial times $Z$-quark couplings, $C_{1 q}$, different from previous experiments. In particular, the experiment makes a high-precision measurement of $2 C_{1 u}+C_{1 d}$. Combined with previous measurements, precise determination of each coupling can be made and is sensitive to physics beyond the Standard Model such as new gauge bosons, supersymmetry, and quark compositeness. 


\section{APPENDIX JLAB SOURCE CONFIGURATION}

As a result of what we learned from the laser room measurements, a new Pockels cell alignment procedure was developed. A detailed alignment procedure was developed for SLAC [44], but JLab's alignment procedure is different because the experimental program demands require slightly different source optics and place significant space constraints on

the laser table. Because the precision of the HAPPEX measurement is unprecedented at JLab, the previous alignment procedure was insufficient to provide the necessary suppression and control of helicity-correlated beam asymmetries. The rest of this section is a detailed description of the JLab polarized-source configuration and Pockels cell alignment procedure we developed for HAPPEX.

\section{A.1 Source Optics Alignment}

The first step in the Pockels cell (PC) alignment procedure is to properly align all optics upstream of the PC. Changes of the optics or their alignment after the PC has been properly aligned can result in larger than desired helicity-correlated beam asymmetries. The laser beam path to the cathode must be established before any optics alignment can take place because any change to the laser beam path through the optics changes the optics' alignment with the beam. The beam path is steered through the optics to the cathode by a JLab Polarized Source Group expert. The position of the laser beam at the cathode is verified using the pick-off beamline to the CCD camera. During the source alignment procedure, the position of the beam on the CCD camera is checked regularly to make sure the beam's path has not changed. Only the Hall A laser is used during the alignment procedure, and all other beams are later aligned to be colinear with the Hall A laser.

Because half-wave plates (HWPs) are specifically designed to provide half-wave retardation for a given wavelength of light and because the two types of cathodes provide high 
polarization at two different wavelengths, it is an obvious but crucial step to ensure the proper HWPs are installed in the beamline before the alignment is done. After these initial steps are completed, we start with the alignment of the most upstream optical element and work our way down the beamline.

\section{A.1.1 Beam Telescope Alignment}

The beam telescope consists of two lenses with a $6.5 \mathrm{~mm}$ focal length mounted on rails so that the distance between the lenses can be adjusted. For this experiment, the telescope was located upstream of the Intensity Attenuator (IA) system. The purpose of the beam telescope is to place the beam waist as close to the helicity PC as possible while maintaining the proper beam spot size at the cathode. The distance between the lenses is tuned empirically by using a Spiricon CCD camera and software to measure the beam spot size at the PC and also at the cathode (using the pick-off beamline). In 2004, we were unable to achieve a waist at the helicity PC while maintaining a small spot at the cathode. The distance between the lenses was $1.3 \mathrm{~cm}$ to achieve a $\sim 500 \mu \mathrm{m}$ spot at the cathode.

\section{A.1.2 IA System Alignment}

The IA system consists of a $\frac{\lambda}{10}$-plate, a Pockels cell, and a linear polarizer (LP). The face of the waveplate is aligned to be as perpendicular to the beam as possible without causing back reflections that would cause problems with the laser lock status. The waveplate is mounted in a rotary stage connected to a picomotor so that the orientation of the waveplate can be adjusted remotely. The orientation of the waveplate sets the dynamic range of the IA feedback as discussed in Section 4.4.

The Pockels cell of the IA system is called the IA cell and has to be aligned such that its optic axis is along the beam direction requiring adjustment of the cell's pitch and yaw angles. The cell was a Lasermetrics $20 \mathrm{~mm}$ cell with windows covering each face of the cell to protect from moisture in the air and index-matching fluid at the window-crystal interface. The cell has an anti-reflective (AR) coating for the range of wavelengths between 600-1000 nm. The cell is mounted on a New Focus 9071 tilt aligner for pitch and yaw angle control. The optic axis can be roughly aligned by using the isogyre method described in [75] 
and more carefully aligned by measuring extinction between crossed LPs. An LP is placed just downstream of the IA cell and the extinction ratio is calculated by measuring the ratio of light transmitted through the LP when it is aligned with the beam's polarization and $90^{\circ}$ (crossed) to it. When no voltage is applied to the IA cell, the indices of refraction along the two axes perpendicular to the optic axis (called the ordinary and extraordinary axes), are essentially the same value which differs from the index of refraction along the optic axis. The linearly polarized laser light passes through the IA cell unaltered when the optic axis of the PC is aligned with the laser beam, and the extinction through the crossed polarizer will be maximized. It is not necessary to set the roll angle of the IA cell.

The alignment of the LP in the IA system is straightforward. It is mounted on a rotation stage such that its polarization axis is parallel to the beam's polarization axis, and the LP face is normal to the incoming beam.

\section{A.1.3 Periscope Alignment}

The periscope is only used to steer the beam to the Gun 3 beamline or to the pick-off beamline with the CCD camera mimicking the cathode position. The periscope consists of two mirrors each of which bends the beam by $90^{\circ}$. The first mirror is on a spring-loaded mount and inserted for using the periscope. This mirror bends the beam to the vertical direction and the second mirror forces the beam back to the horizontal direction where it travels across the accelerator beamline to the Gun 3 laser table (outrigger table). A second periscope on the outrigger table is used to perform the reverse operations to recover the proper beam height. The periscope mirrors must be carefully aligned such that the angle of incidence is $45^{\circ}$ to preserve the linear polarization on the beam.

\section{A.1.4 IHWP Alignment}

The next optical element in the beamline is the insertable half-wave plate (IHWP) which is mounted on a rotation stage connected to a servo-motor. The servo-motor allows the IHWP to be inserted and removed from the beamline remotely. The alignment of the IHWP is such that its face is normal to the beamline taking care that the back reflections do not cause problems with the laser lock. Since a HWP simply rotates the orientation of 
linearly polarized light, transmission through a crossed LP is used to find the proper HWP orientation. An LP oriented with its polarization axis crossed to the incoming polarization is placed downstream of the IHWP. The angle of the IHWP is adjusted until the maximum transmission through the LP is achieved such that the light passing through the IHWP is polarized perpendicular to the incoming polarization.

The degree of linear polarization (DoLP) incident on the PC is measured for the IHWP "in" and "out" states by measuring the minimum and maximum transmission through an LP. Assuming the beam is $100 \%$ polarized,

$$
1=\operatorname{DoLP}^{2}+\operatorname{DoCP}^{2}
$$

where DoCP is the degree of circular polarization. The DoLP is calculated as

$$
\operatorname{DoLP}=\frac{I_{\max }-I_{\min }}{I_{\max }+I_{\min }}
$$

where $I_{\max }\left(I_{\min }\right)$ is the maximum (minimum) transmission through the LP. Typical values for the DoLP incident on the PC are around $99.9 \%$.

Once the IHWP has been aligned, it is placed in the "out" position until it is needed again.

\section{A.1.5 Pockels Cell Alignment Procedure}

The Pockels cell is the optical element which allows parity-violating experiments to rapidly flip the helicity of the electron beam. The PC is used as a quarter-wave plate (QWP) to convert linear polarization to circular polarization and is referred to as the CP PC or helicity PC. Based on the PC characterization done in the laser room, the PC with the best properties for minimizing helicity-correlated beam asymmetries is chosen to be the helicity PC. For our experiment the best cell was a Cleveland Crystals $19.5 \mathrm{~mm}$ QX2035 KD*P cell which we named "Arwen." The new alignment procedure for the helicity PC was developed for the 2004 HAPPEX run and was improved upon for the 2005 HAPPEX run. The alignment procedure has four parts which take several hours to complete: 
1. Align PC pitch and yaw angles using the isogyre method and extinction between crossed polarizers

2. Set PC roll angle and quarter-wave voltages (QWV)

3. Align PC pitch and yaw angles to minimize position differences due to beam divergence $(2005)$

4. Center PC geometrically to minimize position differences due to steering.

\section{Step 1}

The first part of the helicity PC alignment is aligning the pitch and yaw angles of the PC such that the optic axis of the crystal is aligned with the laser beam path. This procedure is the same as that described for the IA cell in Section A.1.2. The remaining steps are described below.

\section{Step 2}

The roll angle of the $\mathrm{PC}$ is set so that the fast and slow axes of the $\mathrm{PC}$ are oriented $45^{\circ}$ to the incoming polarization axis. Because the indices of refraction along the PC's ordinary and extraordinary axes are the same when no voltage is applied to the cell, the polarization is unaffected by the roll angle of the cell in this situation (as long as the optic axis is already properly aligned with the laser beam path). For this reason, the roll angle has to be set while applying high voltage (HV) to the cell and using an LP analyzer downstream of the PC.

The helicity $\mathrm{PC}$ is placed in an aluminum housing attached to a rotation stage equipped with a vernier for fine adjustment of the roll angle. The rotation stage is mounted to an aluminum plate attached to the New Focus tilt aligner. In addition, the PC and mounts are attached to a Line Tool, Co. Model-G triple axis (x, y, z) translation stage as shown in Figure A.1. The stage provides 1" translation with $0.001 "$ precision for all three axes.

For the selection of the PC roll angle and QWV, a spinning linear polarizer (SLP) is placed downstream of the helicity PC for use as an analyzer. The light transmitted through the SLP is incident on a power meter which is readout in an oscilloscope. We align the 


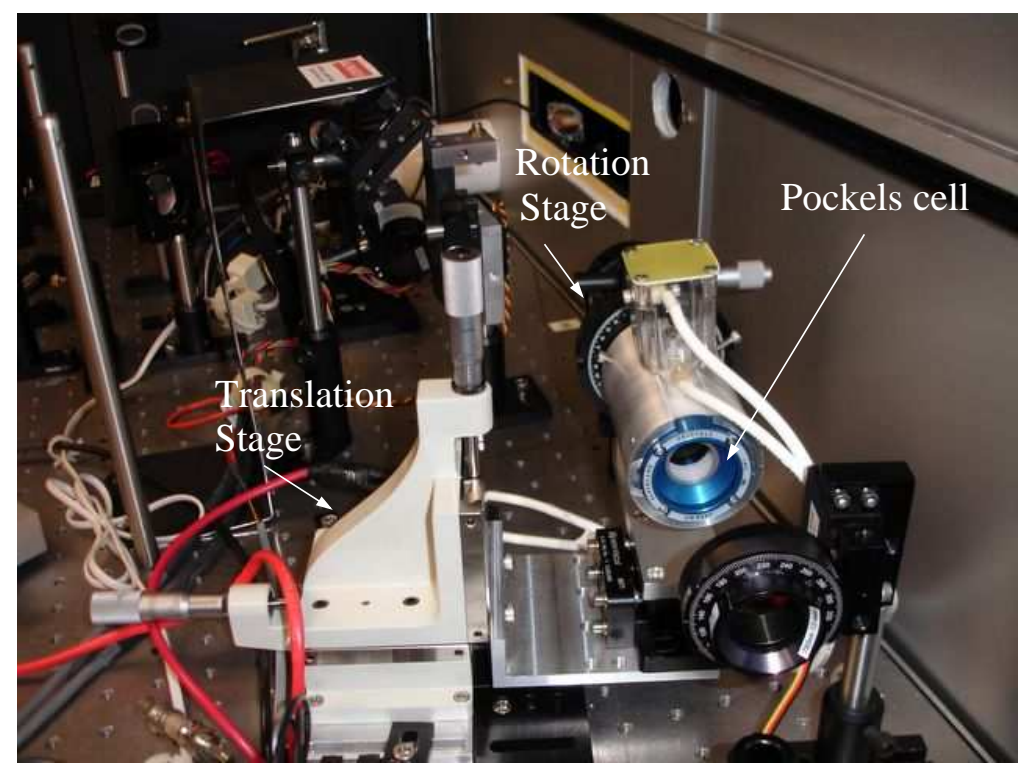

Figure A.1. Picture of helicity Pockels cell (in blue anodized housing) mounted to the rotation and translation stages.

cell using the toggle helicity mode for the HV switch because the static QWV differs from the QWV at $30 \mathrm{~Hz}$. With $\mathrm{HV}$ on the $\mathrm{PC}$, the wave pattern on the oscilloscope is a sine wave. The DoLP is measured according to Equation A.2 such that the sine wave flattens to a straight line as the light incident on the SLP approaches $100 \%$ circular polarization.

The necessary voltage to be applied to the PC to make it behave as a QWP is directly proportional to the laser wavelength. The phase shift on the beam is given by

$$
\delta=\frac{2 \pi}{\lambda} K V_{\delta}
$$

where $\mathrm{K}$ is a property of the crystal and is $90.48 \frac{\mathrm{nm}}{\mathrm{kV}}$ for these PCs, $\lambda$ is the laser wavelength, and $\mathrm{V}_{\delta}$ is the voltage needed to achieve the desired phase shift. Typical voltages for quarterwave retardation are $2.3 \mathrm{kV}$ and $2.5 \mathrm{kV}$ for laser wavelengths of $780 \mathrm{~nm}$ and $850 \mathrm{~nm}$ respectively. The voltage on the PC is controlled through an EPICS interface which sends a DAC voltage between $0-10 \mathrm{~V}$ to the $0-4000 \mathrm{~V}$ PC HV power supplies. In order to set the PC voltages more precisely, the electronics were set up to operate the power supplies over the narrow range of 2200-2800 V for the full range of the DAC (when using $850 \mathrm{~nm}$ light). 
The roll angle and QWV are set using an iterative process. First the roll is set to minimize the SLP oscillation amplitude, and then the voltages are adjusted simultaneously for each helicity state to further decrease the SLP oscillation amplitude. At this point there is usually one helicity state which has significantly more DoLP than the other. The next step is to adjust the roll to better equalize the DoCP between the two states, and then adjust the $\mathrm{HV}$ for each state again to obtain maximum DoCP. The process is continued until no improvement can be made. Generally DoLP $\leq 2.5 \%$ is achievable.

Once the roll angle and QWV have been found for the IHWP "out" state, the IHWP is inserted in order to find the QWV for the "in" state. Because the PC roll angle is optimized for the IHWP "out" state, any change in the orientation of the laser light with respect to the PC ordinary and extraordinary axes for the "in" state must be made by tweaking the roll angle of the IHWP. The voltages used for the "in" state should be fairly similar to the "out" state, but fine adjustments are necessary. The QWV are adjusted to minimize the SLP oscillation amplitude; and if the two helicity states have very different DoCP, the roll angle of the IHWP can be adjusted. In general during this stage of the alignment, the IHWP roll angle has to be only slightly tweaked from the position determined in Section A.1.4 by an amount indeterminable on the rotation stage's scale. Finally, the SLP is removed from the beamline.

\section{Step 3}

Step 3 of the alignment procedure requires an LP analyzer to be inserted in the beamline downstream of the PC and a QPD is installed farther downstream to measure the position differences due to the divergence of the beam. The LP transmission axis should be either parallel or crossed with the laser polarization incident on the $\mathrm{PC}$ for maximum analyzing power. Then QPD $x$ and $y$ translation scans are performed to properly center the beam on the four pads and to simultaneously calibrate the QPD signal response to position. The attenuator setting adjusts the laser power incident on the QPD for optimal dynamic range. Next a PITA scan is performed to measure the sensitivity to the analyzing power, and the PC voltages are adjusted to zero $A_{Q}$. 
In order to minimize position differences due to the the laser beam's misalignment to the optic axis of the PC, step 3 consists of conducting pitch and yaw scans with the IHWP in and out of the beamline. A pitch scan is performed by taking data at the nominal PC position and for several locations over a range of $\pm 4 \mathrm{mrad}$ for the IHWP "out" state and then repeated for the "in" state. The $y$ position differences are only sensitive to the pitch angle such that these two scans should simultaneously determine the pitch angle required to achieve $\Delta y=0$. This procedure is then repeated for the $\mathrm{PC}$ yaw angle. The $x$ position differences are only sensitive to the yaw angle such that these scans determine the yaw angle required to achieve $\Delta x=0$. Following these sets of scans, the pitch and yaw angles are set to the desired values representing the best average alignment of the beam along the PC optic axis, and a measurement of the position differences is done to verify the PC pitch and yaw alignment in both IHWP states. Finally, the IHWP is set to the "out" position and the LP analyzer is removed from the beamline.

\section{Step 4}

Step 4 centers the PC geometrically to minimize position differences due to PC steering effects. The QPD is placed far downstream of the PC to provide a large lever arm for measuring position differences due to steering. A lever arm of $1.3 \mathrm{~m}$ was achieved by using a mirror downstream of the $\mathrm{PC}$ to deflect the beam to an open area on the laser table where the QPD was placed. The laser power incident on the QPD must be adjusted and the QPD recalibrated using the same procedure in step 3. There should be no analyzing power in this setup, but the mirror could have a small effect; therefore, a PITA scan is performed to measure the sensitivity to analyzing power and verify that it is small $(<0.5 \mathrm{ppm} / \mathrm{V})$.

PC $x$ and $y$ translation scans are performed by measuring the position differences at the nominal PC position and at several locations over a range of \pm 0.15 " for the IHWP "out" state. The data are fit to a line, and the PC position is moved to the location which nulls both $x$ and $y$ position differences. Then a measurement of the position differences at this location is completed. If the position differences are satisfactorily small (zero within the error bars), the IHWP is inserted and they are remeasured to verify that they are the same 
size and sign for the "in" state. Otherwise, additional PC $x$ and $y$ scans are performed until the position differences are measured to be sufficiently small in both IHWP states.

Finally, the beamline is setup as it was for step 3, and the position differences due to birefringence gradients are measured for the final PC alignment. Then the LP analyzer is removed from the beamline in preparation for the RHWP alignment

\section{A.1.6 RHWP Alignment}

The Rotatable HWP (RHWP) is placed just downstream of the helicity PC on a rotation stage connected to a stepper motor used for remote rotation control. The RHWP is aligned such that it is normal to the beam. The orientation of the RHWP is set empirically using electron beam data (discussed in Section A.2) in order to minimize the sensitivity to the cathode analyzing power.

\section{A.2 Injector Source Setup}

The RHWP angle and the IA slope are determined using electron beam data from the injector and the hall. The RHWP angle controls the orientation of the residual linear polarization incident on the cathode. It is necessary to setup the RHWP angle using the electron beam to minimize charge asymmetry and position differences due to the interaction of the residual linear polarization with the cathode analyzing power. The IA slope can be measured during the source configuration, but it is not necessary; and by setting it up on the electron beam, it is not invasive to the other experimental halls' data-taking.

\section{A.2.1 IA Setup}

The IA is used to control the charge asymmetry left after the PC voltages and RHWP are tuned to minimize $A_{Q}$. For this reason, the IA slope only needs to be around $100 \mathrm{ppm} / \mathrm{V}$. An IA scan measures how $A_{Q}$ varies with the voltage applied to the IA cell. We chose to run with an IA slope of $\pm 200 \mathrm{ppm} / \mathrm{V}$. We measured the IA slope for different IA waveplate settings until we achieved roughly this value. Because position differences can be induced by the IA system, we measure those slopes as well to verify that they are reasonable $(<0.5 \mathrm{~nm} / \mathrm{V})$. 
The Hall C IA system is used to zero their $A_{Q}$ no matter what size it is; therefore, the only stipulation is that the waveplate angle is such that the IA slope is large enough to achieve this. A typical value during our experiment was $\sim 1500 \mathrm{ppm} / \mathrm{V}$.

\section{A.2.2 RHWP Setup}

There are four main criteria for choosing a RHWP angle:

1. Small $A_{Q}$ in each IHWP state

2. Small, but non-zero PITA slope

3. Small average position differences after IHWP cancellation

4. Small position differences in each IHWP state.

The RHWP angle can be different between the two IHWP states as long as it satisfies the above criteria.

The setup of the RHWP angle requires a series of four RHWP scans. RHWP scans are conducted for both IHWP states for with the nominal PC voltage (PITA voltage $=$

$0)$ to measure $A_{Q}$ and position differences due to phase gradients of the PC. The scans are repeated with a significant PITA voltage $(\sim 200 \mathrm{~V})$ applied to the PC to measure the contribution of cathode analyzing power gradients to $A_{Q}$ and position differences. The RHWP angle for each IHWP state that satisfies the above criteria is selected for production running. 


\section{BIBLIOGRAPHY}

[1] T. D. Lee and C. N. Yang, Phys. Rev.104, 254 (1956).

[2] C. S. Wu et al., Phys. Rev.105, 1413 (1957).

[3] F. J. Hasert et al., Phys. Lett. B46, 138 (1973).

[4] C. Y. Prescott et al., Phys. Lett. B77, 347 (1978).

[5] J. Ashman. et al., Phys. Lett. B206, 364 (1988).

[6] A. O. Bazarko et al., Z. Phys. C65, 189 (1995).

[7] H. L. Lai et al., Phys. Rev. D55, 220 (1997).

[8] J. Ashman et al., Nucl. Phys. B328, 1 (1989).

[9] S. F. Pate et al., Eur. Phys. J. A 24, 89 (2005).

[10] J. Gasser, H. Leutwyler, and M. E. Sainio, Phys. Lett. B253, 252 (1991).

[11] B. Borasoy and U.-G. Meißner, Ann. Phys. 254, 192 (1997).

[12] D. B. Kaplan and A. Manohar, Nucl. Phys. B310, 527 (1988).

[13] M. N. Rosenbluth, Phys. Rev.79, 615 (1950).

[14] C. E. Hyde-Wright and K. de Jager, Ann. Rev. Nucl. Sci. 54, 217 (2004).

[15] S. Galster et al., Nucl. Phys. B32, 221 (1971).

[16] J. Friedrich and T. Walcher, Eur. Phys. J. A 17, 607 (2003).

[17] J. J. Kelly, Phys. Rev. C70, 068202 (2004).

[18] M. J. Musolf et al., Phys. Rep.239, 1 (1994).

[19] K. A. Aniol et al., Phys. Rev. C69, 065501 (2004).

[20] D. T. Pierce and F. Meier, Phys. Rev. B13, 5484 (1976).

[21] Grady Wilson Miller IV, Ph.D. thesis, Princeton University, 2001, Parity-violation in Forward Angle Elastic Electron-proton Scattering.

[22] Bryan Moffit, Ph.D. thesis, College of William and Mary, 2006, Elastic Scattering of Longitudinally Polarized Electrons from ${ }^{4} \mathrm{He}:$ A measurement of $G_{E}^{s}$ at $Q^{2}=0.1 \mathrm{GeV}^{2}$.

[23] Krishna Kumar, Ph.D. thesis, Syracuse University, 1990, Parity Violation in Elastic Electron Carbon Scattering. 
[24] Brian Humensky, Ph.D. thesis, Princeton University, 2003, Probing the Standard Model and Nucleon Structure via Parity-Violating Electron Scattering.

[25] T. Maruyama et al., App. Phys. Lett. 85, 2640 (2004).

[26] M. Baylac and M. Poelker, (2004), http://www.jlab.org/accel/inj_group/docs/2004/superlattice.ppt-1.ppt.

[27] R. A. Mair et al., Phys. Lett. A212, 231 (1996).

[28] W. Barry, Nucl. Instr. Meth. A301, 407 (1991).

[29] W. Barry, J. Heefner, and J. Perry, in Accelerator Instrumentation: 2nd Annual Workshop: Proceedings, edited by E. S. McCrory (AIP, Batavia, Illinois, 1991), pp. 48-74, CEBAF-PR-90-023.

[30] H. Dong et al., in Proceedings of the 2005 Particle Accelerator Conference, edited by C. Horak (IEEE, Knoxville, Kentucky, 2005).

[31] L. J. Kaufman, K. D. Paschke, and R. Michaels, HAPPEX Technical note, http://hallaweb.jlab.org/experiment/HAPPEX/docs/cavity2005.pdf (2005).

[32] R. Michaels, Hall A Status Report - 2004, 41 (2005).

[33] J. Alcorn et al., Nucl. Instr. Meth. A522, 294 (2004).

[34] The Hall A Hypernuclear Collaboration, (1996), A Proposal for Two Septum Magnets for Forward Angle Physics in Hall A at TJNAF.

[35] P. Brindza et al., IEEE Trans. App. Supercond. 11, 1594 (2001).

[36] J. J. LeRose and P. Brindza, Hall A Status Report - 2005, 9 (2006).

[37] R. Michaels, http://hallaweb.jlab.org/experiment/HAPPEX/docs/OSP_sweeper.doc (2005).

[38] A. Vacheret and D. Lhuillier, HAPPEX Technical note, http://hallaweb.jlab.org/experiment/HAPPEX/docs/electron_detector.ps.gz (2002), See also http://www-dapnia.cea.fr/Sphn/Parity/Happex2/Det/index.php.

[39] L. J. Kaufman, D. Lhuillier, and R. Suleiman, HAPPEX Technical note, http://hallaweb.jlab.org/experiment/HAPPEX/docs/PMT_linearity2.ps.gz (2005).

[40] N. Falletto et al., Nucl. Instr. Meth. A459, 412 (2001).

[41] R. Hicks et al., Nucl. Instr. Meth. A553, 470 (2005).

[42] R. Suleiman, A Conceptual Design of Hall A Lumi Monitor (2002).

[43] G. D. Cates et al., Nucl. Instr. Meth. A278, 293 (1989).

[44] T. B. Humensky et al., Nucl. Instr. Meth. A521, 261 (2004).

[45] K. A. Aniol et al., Phys. Lett. B635, 275 (2006).

[46] A. Acha et al., nucl-ex/0609002 (2006). 
[47] J. Grames, Jefferson Lab Technical Note JLAB-TN-05-028 (2005).

[48] T. Holmstrom, R. Feuerbach, and R. Michaels, HAPPEX Technical note, http://hallaweb.jlab.org/experiment/HAPPEX/docs/qsq_2004.pdf (2005).

[49] T. Holmstrom, R. Feuerbach, and R. Michaels, HAPPEX Technical note, http://hallaweb.jlab.org/experiment/HAPPEX/docs/qsq_2005.pdf (2006).

[50] M. J. Musolf and T. W. Donnelly, Nucl. Phys. A546, 509 (1992).

[51] W.-M. Yao et al. (Particle Data Group), J. Phys. G 33, 119 (2006).

[52] S.-L. Zhu et al., Phys. Rev. D62, 033008 (2000).

[53] L. C. Maximon and W. C. Parke, Phys. Rev. C61, 045502 (2000).

[54] Vitaliy Ziskin, Ph.D. thesis, Massachusetts Institute of Technology, 2005, Measurement of the Electric Form Factor of the Neutron at Low Momentum Transfers Using a Vector Polarized Deuterium Gas Target at BLAST.

[55] Y. Goto et al., Phys. Rev. D62, 034017 (2000).

[56] A. Bodek, H. Budd, and J. Arrington, AIP Conf. Proc. (USA) 698, 148 (2003).

[57] K. A. Aniol et al., Phys. Rev. Lett.96, 022003 (2006).

[58] D. T. Spayde et al., Phys. Lett. B583, 79 (2004).

[59] F. E. Maas et al., Phys. Rev. Lett.94, 152001 (2005).

[60] D. S. Armstrong et al., Phys. Rev. Lett.95, 092001 (2005).

[61] R. Young et al., Phys. Rev. Lett.97, 102002 (2006).

[62] K. D. Paschke, Private communication (2006).

[63] N. W. Park and H. Weigel, Nucl. Phys. A451, 453 (1992).

[64] H. W. Hammer, U. G. Meißner, and D. Dreschel, Phys. Lett. B367, 323 (1996).

[65] H. W. Hammer and M. J. Ramsey-Musolf, Phys. Rev. C60, 045204 (1999).

[66] A. Silva, H.-C. Kim, and K. Goeke, Phys. Rev. D65, 014016 (2001).

[67] R. Lewis, W. Wilcox, and R. M. Woloshyn, Phys. Rev. D67, 013003 (2003).

[68] D. B. Leinweber et al., Phys. Rev. Lett.94, 212001 (2005).

[69] D. B. Leinweber et al., Phys. Rev. Lett.97, 022001 (2006).

[70] B. Kubis and R. Lewis, Phys. Rev. C74, 015204 (2006).

[71] D. Beck et al., (2005), Jefferson Lab E05-108 proposal, The G0 Experiment Backward Angle Measurements.

[72] K. D. Paschke et al., (2005), Jefferson Lab E05-109 proposal, A Measurement of Nucleon Strangeness at High $Q^{2}$. 
[73] R. Michaels et al., (2006), Jefferson Lab E06-002 proposal, A Clean Measurement of the Neutron Skin of ${ }^{208} \mathrm{~Pb}$ through Parity Violating Electron Scattering.

[74] R. Carlini et al., (2005), Jefferson Lab E05-008 proposal, The Qweak Experiment: A Search for Physics at the TeV Scale via a Measurement of the Proton's Weak Charge.

[75] R.L. Goldstein, User's Guide for $K D^{*} P$ and Lithium Niobate Qswitches and Modulators for Q-switching, Chopping, and Pulse Extraction, http://fastpulse.com/pdf/eomgenl.pdf. 